\title{
PATTERN FORMATION AND FLOW TO FRACTURE TRANSITIONS IN GRANULAR AND SHEAR THICKENING MATERIALS
}

\author{
A PHD THESIS submitted in fulfillment of \\ the requirements for the award of the degree of \\ DOCTOR OF PHILOSOPHY

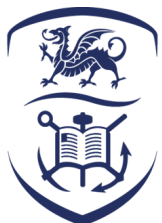 \\ Swansea University \\ Prifysgol Abertawe
}

SUbMitTED B Y

Deren Ozturk

SUPERVISED BY

Bjornar Sandnes

Associate Professor

College of ENGINEERING

SWANSEA UNIVERSITY

Swansea University, UK

NOVEMBER, 2019

Copyright: The author, Deren Ozturk, 2020. 


\section{Declaration}

1. This work has not previously been accepted in substance for any degree and is not being concurrently submitted in candidature for any degree.

2. This thesis is the result of my own investigations, except where otherwise stated. Other sources are acknowledged by footnotes giving explicit references. A bibliography is appended.

3. I hereby give consent for my thesis, if accepted, to be available for photocopying and for inter-library loan, and for the title and summary to be made available to outside organisations.

\section{Deren Ozturk:}

Date: 
This work is dedicated to my father, Hulusi Öztürk;

he taught me what dedication is. 
"Be like water making its way through cracks. Do not be assertive, but adjust to the object, and you shall find a way around or through it. If nothing within you stays rigid, outward things will disclose themselves. Empty your mind, be formless. Shapeless, like water. If you put water into a cup, it becomes the cup. You put water into a bottle and it becomes the bottle. You put it in a teapot, it becomes the teapot. Now, water can flow or it can crash. Be water, my friend."

-Bruce Lee 


\section{Abstract}

The conditions under which a mixture of water and grains will fracture like a solid, rather than flow like a liquid, is the subject of this thesis. Flow to fracture transitions in saturated granular materials are relevant to numerous geological and engineering environments, including magma cavern activity, methane venting on seabeds, carbon dioxide storage, food processing, and innovations in body armour. To examine the flow to fracture transition, two systems are considered. The first is gas-driven fracturing of settled granular media, a slow creeping process that forms labyrinthine patterns. The second is gas-driven fracturing of suspended cornstarch particles, a system which exhibits fascinating "discontinuous shear thickening" behaviour, a topic of much debate in literature. Both systems are subject to experiments within a Hele-Shaw cell, which enables the visualisation of pseudo-2D invasion flow or fracture patterns. Image analysis performed on these patterns led to the application of theories that can predict their behaviours. Fracture formation is found to be a friction dominated process. The invading pressure pushes on the local grains while surface tension of the receding water pulls on them until frictional forces become strong enough to maintain a front, forcing the pressure to disturb grains elsewhere, and in doing so extend and branch the fractures forming a patterned network. Various parameter studies are performed to uncover the variables that determine why a mixture might flow or fracture. Interestingly, the first system is found to transition from fracturing to flowing with increasing pressures, whilst the second system is found to do the opposite. 


\section{Acknowledgements}

I would like to begin by acknowledging what a fantastic supervisor Bjørnar Sandnes has been for me. Thank you, truly, Bjørnar. Your patience has been unbounded, your advice has been invaluable, and you taught me how to get excited about sand and cornstarch (and why everyone else should be too!). Your philosophy and approach to science and research has been a positive influence on me both inside and outside of the lab.

I want to thank Martin Monloubou, James Campbell, David James, and Miles Morgan, as the rotating cast of Bjørnar's Post Docs, for all of the assistance they gave me in the lab and in navigating university bureaucracy. Additional thanks to Emilio Garcia, Rob Phillips, Ali Al-Zuhairi, and Dawang Zhang for being great fellow travellers on the $\mathrm{PhD}$ journey. Most of you beat me to the end, good luck for yours Dawang. I want to thank my old physics crew from York as well: Bieniek, Jack, and Laura for showing how we could go about doing PhDs, and Jess, Alistair, and Alex for helping me get through university in the first place. Many thanks also to Kurt Samson for being a great source of intellectual stimulation (a.k.a. a reliable and necessary distraction).

To Jasmine, thank you for the dedication that is required to marry a PhD student. We have to assume that I'm a long term investment. Thank you for your never ending motivation and irreplaceable ability to listen to any problem that I need to solve and immediately inspire me to try a new solution without having to say a word.

To my Mum, thank you for your continuous encouragement and interest in my work. It really does mean a lot when your Mum insists on spending her evenings checking your thesis for typos. Thank you for all that you've done for me. Thank you to my Dad, who supported me in all my endeavours, especially when he didn't consider them optimal career choices. He passed away just as I started my PhD but I know he would have enjoyed seeing what came of it. Don't worry, I'll get a real job one day (probably). To my Brother, thank you for constantly reminding me that I can't do maths. To my Grandad and Dede, you were the ones that helped instill in me such strong curiosity in how the world works, I treasure that.

Lastly, but not least of all, I would like to sincerely thank the rest of my family, the rest of my friends, and anyone I ever met that asked me what I do for a living and then attentively listened to me explain the rheological dynamics of cornstarch to them. 
My research was funded and supported by the Engineering and Physical Sciences Research Council and Sêr Cymru National Research Network in Advanced Engineering, for which I'm very grateful. 


\section{List of Papers}

D. OZTURK AND B. SANDNES.

A Study of Three-Phase Fracturing in Granular Media using High-Speed Imaging. Journal of Porous Media, Vol. 22 Issue 8, 2019.

Reproduced in Chapter 4.

J. M. CAmpbell, D. Ozturk, And B. SAndnes.

Gas-Driven Fracturing of Saturated Granular Media.

Physical Review Applied, 8 064029, 2017.

Reproduced in Chapter 5.

D. Ozturk, M. L Morgan, And B. SAndnes.

Flow-to-Fracture Transition and Pattern Formation in

a Discontinuous Shear Thickening Fluid.

In preparation.

Reproduced between Chapters 6 and 7 . 


\section{Contents}

Title Page

Declaration ii

Dedication iii

Abstract v v v v

Acknowledgements

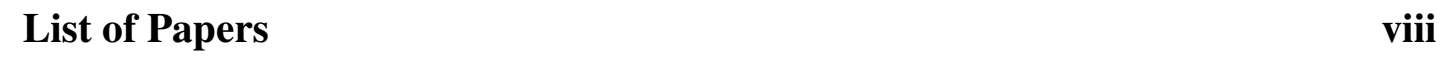

\begin{tabular}{lll}
\hline & Introduction & 1
\end{tabular}

1.1 Pattern Formation in Nature $\ldots \ldots \ldots \ldots$

1.1.1 Natural Non-Biological Patterns . . . . . . . . . . . . 3

1.1 .2 Granular Media . . . . . . . . . . . . . . . . 4

1.2 Multiphase Invasion Patterns and Fracturing . . . . . . . . . . . . . 4

1.2.1 The Hele-Shaw Cell and Invasion Patterns . . . . . . . . . . . . . 5

1.2 .2 Fracturing Fluids . . . . . . . . . . . . . . . . 6

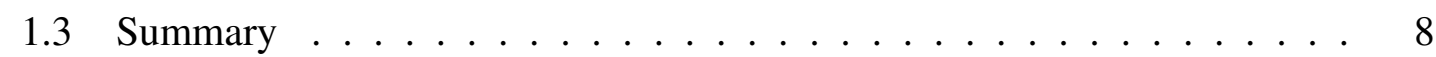

\begin{tabular}{|lll}
\hline 2 & Fundamental Physics & 9
\end{tabular}

$2.1 \quad$ Settled Granular Physics $\ldots \ldots \ldots$. . . . . . . . . . . . . . 9

2.1 .1 Darcy's Law . . . . . . . . . . . . . . . . . . 11

2.1 .2 Kozeny-Carman Equation . . . . . . . . . . . . . . 12

2.1 .3 Surface Tension . . . . . . . . . . . . . . . . . . . . 13

2.1 .4 Young-Laplace Equation . . . . . . . . . . . . . . . . 13

2.1 .5 Contact Angle . . . . . . . . . . . . . . . . . 14

$2.1 .6 \quad$ Friction and the Janssen Effect . . . . . . . . . . . . . . . . . 15

2.2 Suspended Granular Physics ～. . . . . . . . . . . . . . . . . . 18

$2.2 .1 \quad$ Viscosity . . . . . . . . . . . . . . . . . . 18

$2.2 .2 \quad$ Viscosity of a Suspension $\ldots \ldots \ldots \ldots$ 
2.2 .3 Non-Newtonian Fluids $\ldots \ldots \ldots$. . . . . . . . . . . . 21

3 Literature Review 23

3.1 Fracturing in Settled Granular Media . . . . . . . . . . . . . . . . . . 24

3.1 .1 Carbon Capture Storage . . . . . . . . . . . . . . . . . . . . . . . . . . . . . . 25

3.1 .2 Methane Venting . . . . . . . . . . . . . . . 26

3.1 .3 3D model systems . . . . . . . . . . . . . . . . . 27

3.1 .4 Three-phase Hele-Shaw Experiments . . . . . . . . . . . . . . 30

3.1 .5 Hele-Shaw Fracturing . . . . . . . . . . . . . . . 31

3.2 Fracturing in Suspended Granular Media . . . . . . . . . . . . . . . 33

$3.2 .1 \quad$ Magma Flows . . . . . . . . . . . . . . . . . . 33

3.2 .2 Soft Body Armour . . . . . . . . . . . . . . . . . 34

3.2 .3 Rheology of Suspensions . . . . . . . . . . . . . . . . . 36

3.2.4 Proposed Mechanisms for Discontinuous Shear Thickening . . 37

3.2 .5 Friction Transition . . . . . . . . . . . . . . . . . 38

3.2 .6 Complex Suspensions and Hele-Shaw cell . . . . . . . . . . . . 40

4 Fracture Formation at the Granular Scale 43

4.1 Introduction . . . . . . . . . . . . . . . . . . . . 43

4.2 Materials and Methods . . . . . . . . . . . . . . . . . . . 44



$4.2 .2 \quad$ Fracture Pattern Image Analysis . . . . . . . . . . . . . . 45

$4.2 .3 \quad$ High-speed Setup . . . . . . . . . . . . . . . . . . . . . . . 48

$4.2 .4 \quad$ High-speed Image Analysis $\ldots \ldots \ldots$

4.3 Results and discussion $\ldots \ldots \ldots \ldots$. . . . . . . . . . . . 50

$4.3 .1 \quad$ Fracture Pattern . . . . . . . . . . . . . . . 50

4.3 .2 Growth of a Single Fracture . . . . . . . . . . . . . 51

4.3 .3 Deformation of the Granular Bed . . . . . . . . . . . . . . 54

4.3 .4 Compaction Fronts as a Constraint on Pattern Formation ～. . . . 56

4.4 Conclusion . . . . . . . . . . . . . . . . . . 58

5 Parameters of Fracture Pattern Formation 62

$5.1 \quad$ Introduction . . . . . . . . . . . . . . . . . . . 62

5.2 Materials and Methods . . . . . . . . . . . . . . . . 63

$5.2 .1 \quad$ Experimental Setup . . . . . . . . . . . . . . . . . 63

5.2 .2 Data and Image Analysis . . . . . . . . . . . . . . . . . 64

5.3 Results and Discussion . . . . . . . . . . . . . . . . . . 65

5.3.1 Fracture Growth in the Frictional Regime at Low Injection Rate . 65

5.3 .2 Granular Compaction Fronts . . . . . . . . . . . . . . . . . 67

5.3 .3 Effect of Grain Size $\ldots \ldots \ldots 71$ 
5.3 .4 Effect of Granular Friction (grain shape) . . . . . . . . . . . . . 73

5.3 .5 Analytical model for fracture density . . . . . . . . . . . 75

5.3 .6 Dependence on Gas Injection Rate . . . . . . . . . . . . . . . 77

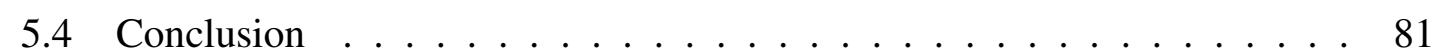

\begin{tabular}{|lll}
6 & Rheology of Shear Thickening Suspensions & 84
\end{tabular}

6.1 Introduction . . . . . . . . . . . . . . . . . . . . . 84

6.2 Rheology Methods . . . . . . . . . . . . . . . . . . 85

6.2 .1 Cornstarch Suspensions . . . . . . . . . . . . . . 85

6.2 .2 Experimental Challenges of Rheology . . . . . . . . . . . . 87

6.2 .3 Experimental Method . . . . . . . . . . . . . . . . 88

6.3 Rheology Measurements . . . . . . . . . . . . . . . . . . . . 90

6.4 Theory . . . . . . . . . . . . . . . . . . . . . 90

6.4 .1 Frictionless to Frictional . . . . . . . . . . . . . . . . . 9 91

6.4 .2 Shear Stress . . . . . . . . . . . . . . . . . . . 92

6.4 .3 Viscosity . . . . . . . . . . . . . . . . 93

6.4 .4 Shear Rate . . . . . . . . . . . . . . . . . 93

6.5 Results and Discussion . . . . . . . . . . . . . . . . . . . 94

6.5 .1 Theoretical Curves . . . . . . . . . . . . . . . . . 9 94

6.5 .2 Going Dimensionless . . . . . . . . . . . . . . 95

6.5 .3 Comparison . . . . . . . . . . . . . . 96

6.6 Conclusion $\ldots \ldots \ldots \ldots \ldots \ldots \ldots$

\begin{tabular}{lll}
\hline 7 & Fracturing in a Discontinuous Shear Thickening Fluid & 99
\end{tabular}

7.1 Introduction . . . . . . . . . . . . . . . . . . . 99

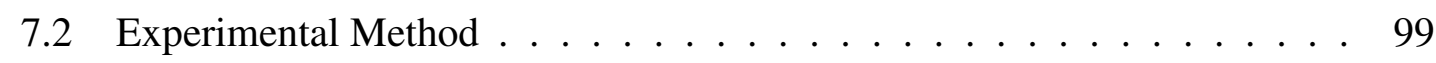

7.3 Results and Discussion . . . . . . . . . . . . . . . . . . 103

7.3.1 Hele-Shaw Flow and Pattern Formation . . . . . . . . . . . 103

7.3 .2 Discussion . . . . . . . . . . . . . . . . . 104

7.4 Conclusion . . . . . . . . . . . . . . . . . . . 107

8 Conclusion 109

$8.1 \quad$ Objectives . . . . . . . . . . . . . . . . . . . . . . . 109

$8.2 \quad$ Fracture Patterns in Settled Grains . . . . . . . . . . . . . . . 110

$8.3 \quad$ Fracture Patterns in DST Suspensions . . . . . . . . . . . . . . . 111

8.4 Comparing Systems $\ldots \ldots \ldots$. . . . . . . . . . . . . . . . . 112

8.5 Open Questions . . . . . . . . . . . . . . . . . . . . . . . 112 


\section{Chapter 1}

\section{Introduction}

This thesis is a study of flow-to-fracture transitions in multiphase granular invasion patterns. In this introductory chapter I will break down what that particular group words mean and why they are of interest. First, I will discuss the study of pattern formation in general, followed by patterns in granular materials specifically. Second, I will outline what multiphase systems are and how they can lead to invasion patterns. Third, I will describe what is meant by fracturing in the context of this work and describe the systems that will be studied. Finally, I will outline the structure for the remainder of this thesis.

\subsection{Pattern Formation in Nature}

A pattern is a form with self-referential regularity. Those forms may appear in physical objects or immaterial information. Our fascination with patterns has an evolutionary basis, linked to mastering our environments and planning for events [1, 2]. A side effect of this evolved interest in patterns is that we can find them beautiful, something that has personally motivated the work in this thesis.

From the tessellations of M. C. Escher, to the looping melodies in Pachelbel's Canon, repeating patterns are often included to make objects, architecture, art, and music more appealing, see Figure 1.1. Patterns are also engineered to provide utility, such as in street layouts for modern cities, intuitive user interfaces on smart-devices, and for efficient supporting structures in buildings. Many of these patterns resemble, or are directly inspired by, forms found in biology. Evolution has solved many problems: for example, zebras evolved stripe patterns to hinder the pattern recognition of predators, bees build their hives with tessellating hexagonal honeycombs, and trees grow in space-filling fractal patterns to maximise access to resources, see Figure 1.1 . 


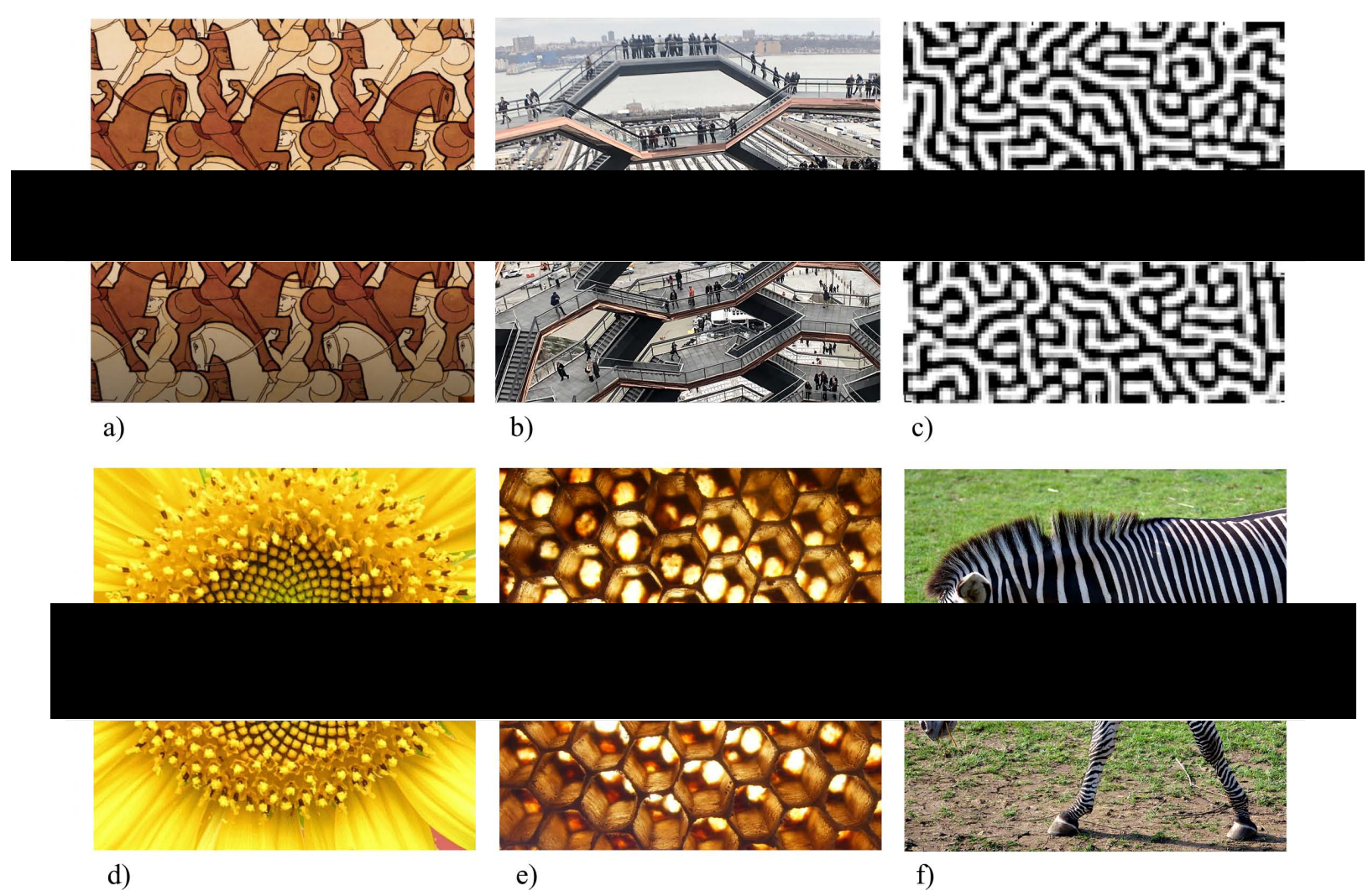

Figure 1.1: Man-made patterns and biological inspirations. a) Horseman by M. C. Escher. b) The Vessel in New York City designed by Thomas Heatherwick. c) A mathematically generated Turing pattern by Tony Kaap. d) Photo of Sunflower florets arranged in a Fibonacci spiral by L. Shyamal. e) Photo of an abandoned honeycomb in Thailand by S. Hoyland. f) Photo of Zebra stripes by A. Karwath.

Since patterns are self repeating, their dynamics and geometries have been deconstructed with mathematics throughout history. Describing harmonies of music and 2D shapes with mathematics in around $500 \mathrm{BC}$, Pythagoras reportedly believed that geometric patterns were the basic constituents for all existence [3]. In the 1500s Leonardo da Vinci noted that leaves are arranged in geometric spiral patterns [4]. Explaining the pentagonal form of some flowers in the 1600s, Johannes Kepler was the first to use the Fibonacci sequence to explain a natural pattern [5]. In 1952, Alan Turing theorised oscillating reaction-diffusion equations that could produce and explain how stripe and spot patterns (dubbed "Turing Patterns") arise on an animal from their homogeneous embryonic state, inspiring much work in modern mathematical biology, see Figure 1.1 [6, 7]. 


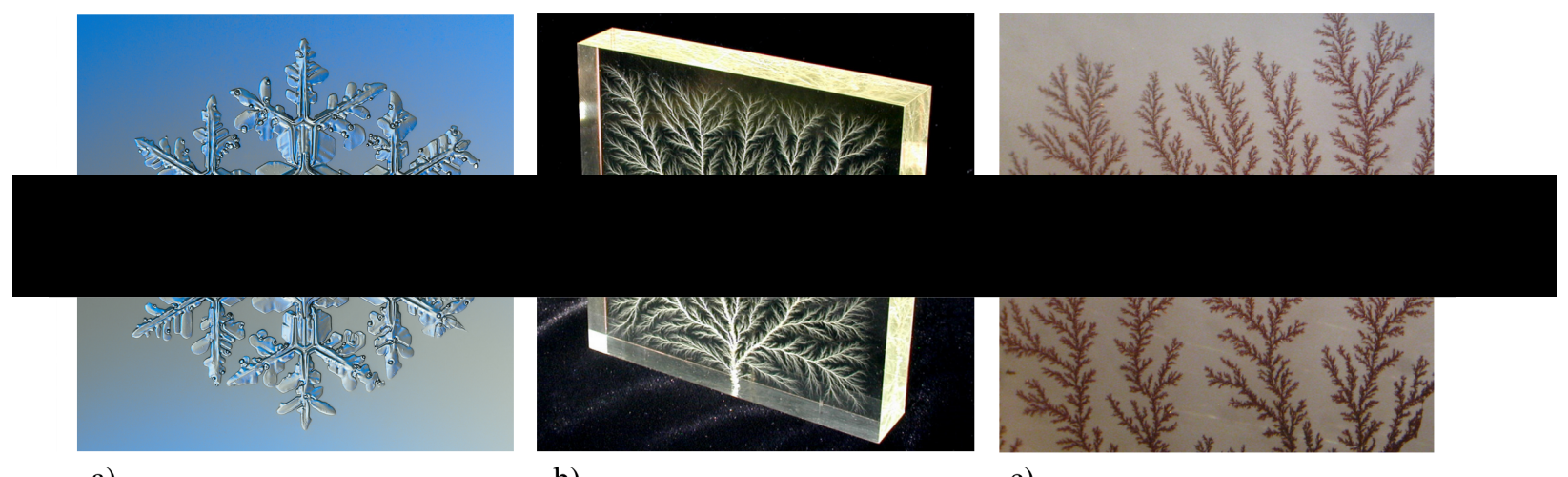

a)

b)

c)

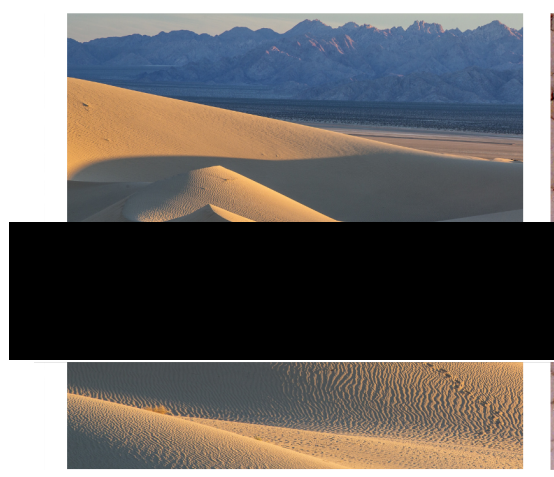

d)
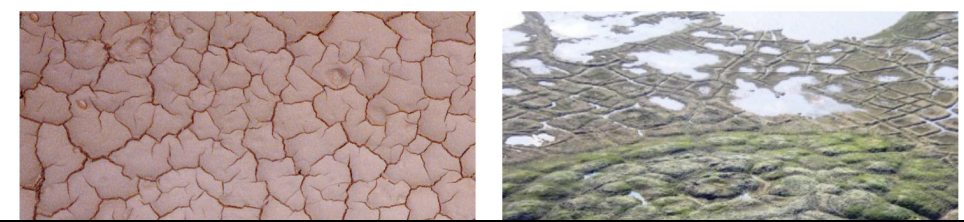

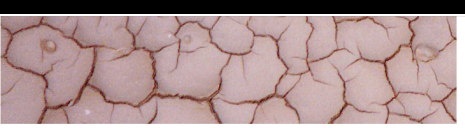

e)

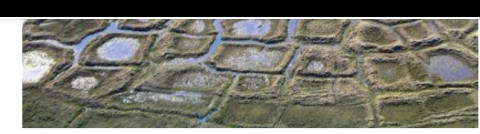

f)

Figure 1.2: Natural non-biological patterns. a) Photo of a snowflake illustrating natural symmetries by A. Kljatov. b) Lichtenberg figure in a block of plexiglas by B. Hickman. c) Photo of dendritic copper crystals by J. Werther. d) Photo of Cadiz Dunes Wilderness, California by B. Wick. e) Photo of mud cracks by I. Alexander. f) Photo of melting pingo and polygon wedge ice near Tuktoyaktuk, Canada by E. Pike.

\subsubsection{Natural Non-Biological Patterns}

All of the patterns described so far are formed with agency, made for a purpose at some cost to the biological maker. But there are also patterns in nature that spontaneously assemble despite having no agent or purpose, seemingly in defiance of entropy. Super-cooled water droplets in clouds amalgamate around a dust particle to freeze into snowflakes with intricate symmetries, winds rolling across deserts build up sand into repeating dunes, and running rain water taking paths of least resistance form fractal river networks, see Figure 1.2 a) and d). These natural but non-biological patterns form as a result of balances being repeatedly struck between competing forces. When their contributing physical mechanics are adequately understood, predictions can be made regarding the formation of such structures. In a mirrored fashion, when presented with a pattern that has ceased evolving, its past state can be approximated by figuring out what competing physical mechanics would have been required to form it. 
As these patterns self assemble through balancing mechanics, where ever mechanisms compete in a similar fashion the same patterns can emerge despite being formed in different mediums or spatial scales. For example, spheres form where the balance between two opposing omni-directional forces is critical, i.e air bubbles (thermal pressure opposed by surface tension) and stars (radiation pressure opposed by gravity). Fern-like branching patterns occur when a pressure gradient over a medium is amplified at perturbations in the forefront of its growth, i.e. Lichtenberg figures (voltage gradient over a conductive surface that increases the closer it gets to an earth) and dendritic crystal growth (mineral concentration gradient in solution over stone that is driven by diffusion from behind its growth), see Figure 1.2 b) and c).

\subsubsection{Granular Media}

Grains are an almost ideal source of natural non-biological patterns: they pile up with friction, which means some formed patterns are structurally stable while others are not; they are too large to be displaced by thermal agitation, which eliminates a large source of noise from their systems; they are discrete objects found in large groups, leading to macroscopic behaviour that allows them to "flow" like a fluid, which encourages pattern dynamism. These characteristics all contribute to the formation of natural patterns like the sand dunes mentioned earlier, as well as river meanders, mud cracks, and periglacial patterned grounds, see Figure 1.2 d), e), and f).

\subsection{Multiphase Invasion Patterns and Fracturing}

All of the dynamic granular patterns mentioned above are made with granular media that is subject to flowing fluids. It is the balance struck from interacting forces between the grains and fluids that results in the patterns and their dynamism. It is also the interplay between a multitude of mobile particles as well as one or more fluids that make modelling these systems so complex. This thesis will focus on multiphase granular systems, where solid, liquid, and gaseous phases all interact. Specifically patterns formed when a gas is forced into a system filled with a granular media submerged in a liquid. This may read like a narrow circumstance, but it is a process that is relevant to numerous geological and industrial processes, such as gas release in magma caverns [8, 9], methane venting on seabeds [10, 11], carbon dioxide storage in geological formations [12, 13], and gas-driven fracturing for enhanced remediation of contaminated soils [14, 15]. 
As a result of their relevance to nature and industry, multiphase granular systems are of interest to physicists, mathematicians, geologists, industrial engineers, and environmental engineers. However, since these processes often occur underground, they are also difficult to study as the fashion in which the gas invades into the granular-liquid mixtures is difficult to observe. A technique recently developed using high-speed synchrotron $\mathrm{x}$-ray microtomography has enabled real time imaging of displacement events within porous rock [16]. Despite this method utilising expensive equipment, it can only observe a volume of approximately $1 \mathrm{~mm}^{3}$ in size. As imaging real world large scale multiphase granular events is still not feasible, researchers create model systems that grant a window into the flow patterns occurring.

\subsubsection{The Hele-Shaw Cell and Invasion Patterns}

One such system is the Hele-Shaw cell. Invented by Henry Selby Hele-Shaw (who was born in 1854 in my hometown of Billericay, Essex), the Hele-Shaw cell is a simple setup made from two parallel glass plates narrowly separated to form a channel through which fluids can flow whilst the researcher can observe the flow patterns in 2D through the transparent glass [17]. Hele-Shaw cells frequently have either a rectangular design with an injection inlet at one of the narrower sides or a circular design with an injection inlet in the centre; both geometries will be used in this thesis.

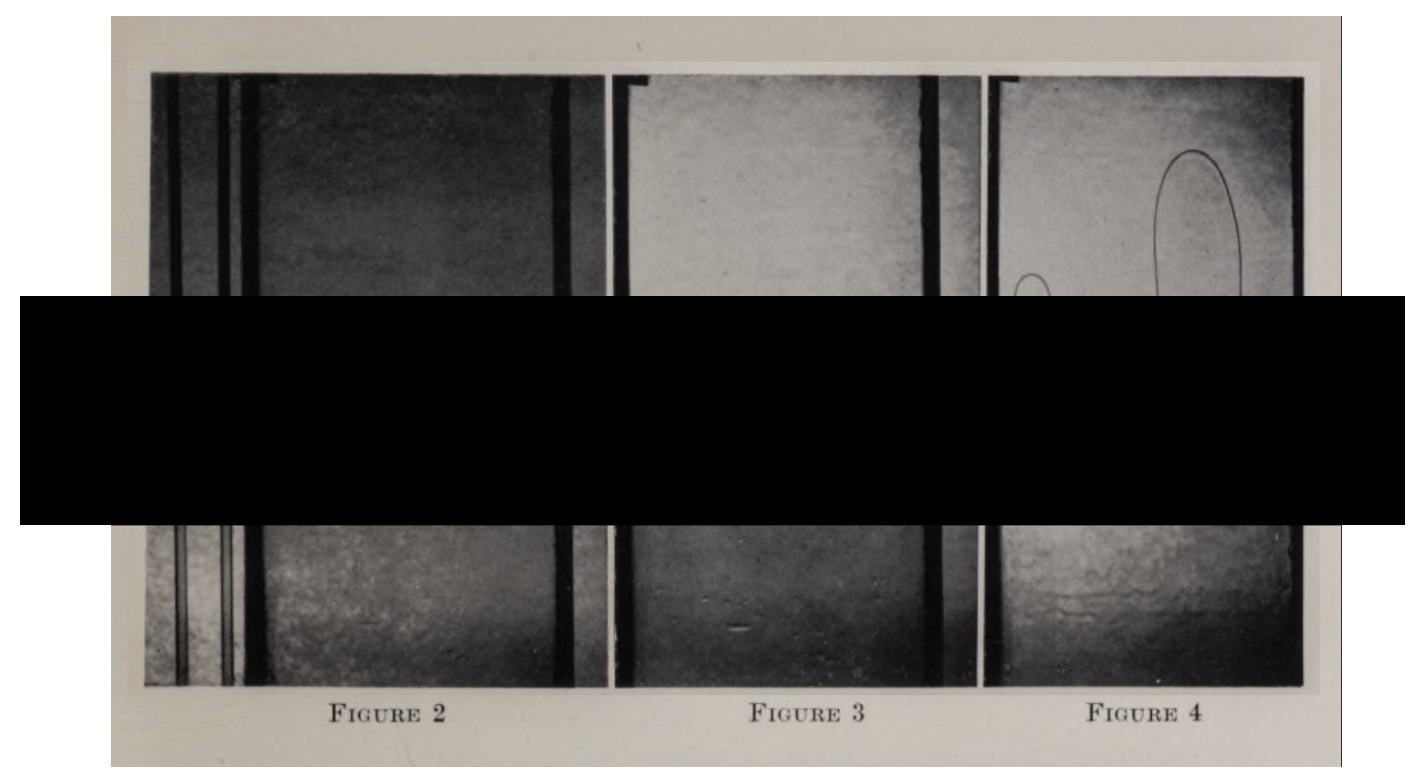

Figure 1.3: Viscous fingering patterns by Saffman-Taylor [18]. Air (less viscous) invades glycerine (more viscous) within a Hele-Shaw cell to form viscous fingering instabilities. 
Among the first uses of the Hele-Shaw cell was a study in 1958 by Phillip Geoffrey Saffman and Sir Geoffrey Ingram Taylor of flow patterns observed between two imiscible fluids (fluids that will not mix) with different viscosities [18, 19]. If the fluid with the lower viscosity occupies the cell while the higher viscosity fluid is injected, the invasion pattern has a stable front (straight in a rectangular cell and circular in a circular one). However, in the reverse case, the lower viscosity fluid injects with an unstable front that develops into finger-like structures that push into the higher viscosity fluid. This instability is named the Saffman-Taylor instability in their honour while the fingers are referred to as viscous fingers, see Figure 1.3 .

Though not its focus, for the context of this work, it is important to note that the Saffman-Taylor instability and its viscous fingers will form when air is injected into water. It can also occur under some circumstances when the Hele-Shaw cell contains a granular material in it in addition to the water, circumstances for which will be discussed and recreated later in this thesis, see Figure 1.4.

\subsubsection{Fracturing Fluids}

The focus of this thesis is the circumstances under which multiphase granular invasion patterns result in fractures. A necessary distinction needs to be made with how the "fracturing" that is studied in this work is fundamentally different to more conventional fracturing of elastic and cohesive materials such as metals and rocks, where a previously continuous solid phase is separated due to stress. In the granular fracturing process described here, though they are all individually solid, there are no cohesive bonds between the grains. It will be shown that the fracture-like shape of the invasion pattern is a result of pressure and surface tension forces acting on the fracture interface, frictional forces acting between grains, and frictional forces acting between grains and the confining boundaries to hold back the grains into the form of narrow cavities that resemble fractures. The fractures observed here are similar to mudcracks, where drying causes the granular material to split as the water tension from the receding water interface pulls on it.

The invasion of air into a liquid which is partially filled with granular material and confined in a pseudo-2D system results in a particularly rich set of patterns, including fractures, viscous fingers, labyrinthine structures, and bubbles, as can be seen in Figure 1.4 [20-23]. The patterns formed are dominated by fronts of dense granular material which accumulate ahead of interfaces, retarding advancement by means of friction. In contrast, if the liquid contains a granular material which is held rigidly in place then "capillary" invasion is observed between the grains or "viscous fingering" at very high injection rates [24-27]. Fracturing occurs between these two extremes: when the liquid is fully packed with granular material but not so tightly packed that the grains are immobile. This fracturing process results in intriguing narrow branching structures, as shown in Figure 1.4 . 


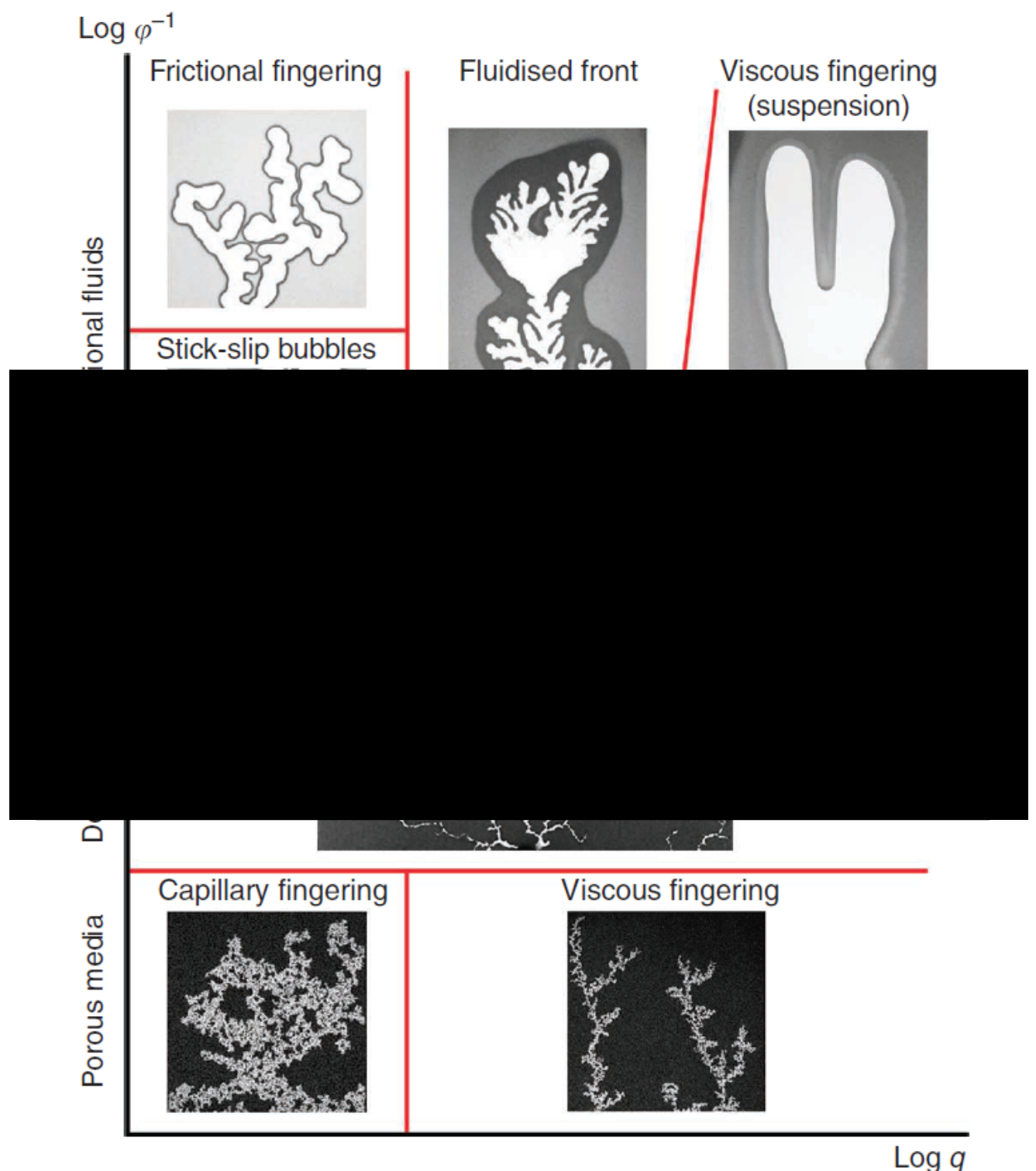

Figure 1.4: Phase diagram of granular Hele-Shaw cell invasion patterns by Sandnes et al. [21]. The phases are roughly classified in relation to $q$, the injection rate of the invading air, and $\phi^{-1}$, the inverse of the fraction of the cell height that is occupied by grains i.e. the cell is fully packed at the bottom of the diagram and minimally packed at the top.

The work presented here will be split between two systems. The first is gas-driven fracture patterns in settled granular media. Here glass beads that are denser than water are poured into a water saturated rectangular Hele-Shaw cell and the grains are allowed to settle out of suspension under gravity before air is injected to make branching fracture patterns. The second is gas-driven fracture patterns in suspended granular media. Here cornstarch particles are mixed with water to form a milky suspension which is poured into a circular Hele-Shaw cell before air is injected to make branching fracture patterns as well as viscous fingering patterns depending on the viscous response of the suspension. The characteristics of the patterns formed in each of these systems will be analysed, the processes through which they form will be discussed, and models that describe their behaviour will be presented. 


\subsection{Summary}

In this chapter, the experimental setups that will be examined in this thesis have been introduced through a broader consideration of the study of pattern formations. Chapter 2 outlines the established physics and mathematical models that are foundational for the theories that will be presented later. Chapter 3 forms a literature review of modern research to contextualise the niche that this work fills. Chapter 4 presents an analysis and simple model of how settled granular fracture patterns form using a high-speed camera. Chapter 5 experiments with how the properties of the settled grains and the gaseous injection rate influence the fracture pattern characteristics, a model to describe the changing behaviour is also introduced. Chapter 6 consists of a rheological study of the solid-like discontinuous shear thickening behaviour of cornstarch suspensions as well as introducing the Wyart-Cates model used to explain it. Chapter 7 presents a phase diagram of a variety of cornstarch suspension invasion patterns, including fracturing, in the framework of the Wyart-Cates model. Finally, Chapter 8 will conclude the findings presented in this thesis while discussing questions it raises for future work to pursue. 


\section{Chapter 2}

\section{Fundamental Physics}

This chapter lays a foundation of physics and models that will be utilised for work presented in later chapters. It will begin by discussing fluid flows in static settled granular packings, then surface tension physics with the introduction of a gaseous phase, followed by physics for a deformable granular packing. In the second section fluid flow in suspended granular media will be discussed, outlining how the viscous response of complex non-Newtonian fluids is categorised.

\subsection{Settled Granular Physics}

For a fluid to flow through a solid medium there needs to be space within the solid for it to flow, called its pore space. A material's porosity describes how much of it is empty to pore spaces, with its effective porosity being how much is available for fluid flow. Effective porosity $(n)$ is then defined as the ratio between the volume of available pore space $\left(V_{P}\right)$ and the total volume of the porous medium $\left(V_{T}\right)$. The complement of this value is the filling fraction of solid material $(\phi)$ i.e. the ratio of how much a total volume is occupied by a solid:

$$
\phi=1-n=1-\frac{V_{P}}{V_{T}} .
$$

The packing fractions for a porous medium made of spherical particles (a granular packing) have been well studied for hundreds of years since the efficient packing of spheres has mercantile applications [28, 29]. The most efficient packing for equal spheres has a hexagonal geometry with $\phi_{\max }=\sqrt{2} \pi / 6 \approx 0.74048$. 
For a granular material, its bulk packing fraction is influenced by the process by which it is packed, a number of its material qualities, as well as the geometry of its container. Packing fractions range from 0.55 to 0.64 for random packings of spheres poured into a container [30-33]. The $\phi=0.64$ upper limit is reached by tapping or vibrating the container alongside compressing the packing, this is referred to as a Random Close Packing (RCP). With no tapping or compression $\phi \approx 0.62$ [32]. The lower limit for a granular packing that can support the most void space and yet remain stable is referred to as a Random Loose Packing (RLP). RLP depends on grain surface roughness and the specific preparation method, e.g. slow sedimentation in near density-matched fluid [33]. Grain surface roughness increases inter-granular friction, supporting higher tangential forces that stabilise the packings at lower $\phi$ values. For non-spherical grains the idea of "random" packings begins to break down: non-spherical particles will have an orientation bias with respect to each other during sedimentation [34]. In simulations, ellipsoids with aspect ratios from 1.0 to 0.7 have been found to have an increasing RLP fraction when settling [34].

In natural systems, as well as the experiments performed in this thesis, the granular materials have a distribution in sizes (polydisperse). This results in a higher RCP as smaller grains can occupy voids made by larger grains. For example, Sohn and Morland (1968) [35] report $\phi_{R C P} \approx 0.7$ for Ottowa sand that has a Gaussian size distribution with a dimensionless standard deviation of $\sigma / \bar{x} \approx 0.6$. $\phi_{R C P}$ increases with the spread of the grain size distribution function (polydispersity); the shape of the distribution function also has a significant effect on $\phi_{R C P}[36]$.

The container of a granular material reduces the average $\phi$ of the packing due to edge effects caused by frustrated packings associated with the boundaries of the container. Typically, the size of the frustrated zone scales with grain diameter $d$ in monodisperse packings [30, 37, 38]. Using Desmond and Weeks (2009) [38] a simplified model can be described for confinement effects in a planar geometry (Hele-Shaw cells, fractures). Figure 2.1 shows the cross-section between two parallel confining walls separated by a gap $b$. The central region is assumed to have a packing fraction $\phi_{b u l k}$ which is representative of the packing in an infinite system. The packing takes a lower value of $\phi_{\text {wall }}$ within each zone near the two walls, the size of these zones scales with the particle diameter $c d / 2$ at both sides (where $c$ is a dimensionless scaling factor). The weighted average gives the overall packing fraction [38]:

$$
\phi=\phi_{b u l k}-\frac{c d}{b}\left(\phi_{b u l k}-\phi_{w a l l}\right)
$$




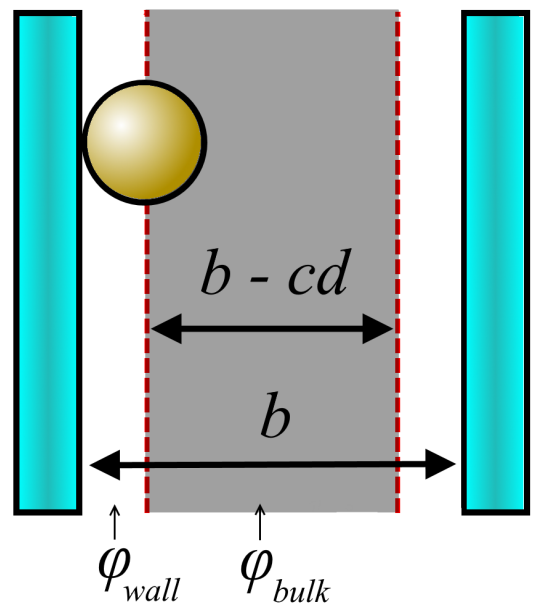

Figure 2.1: Diagram illustrating confinement effects upon packing fraction. $b$ is the distance between the confining plates, $d$ is the diameter of the grain, and $c$ is a scaling factor. The packing fraction in zones near the confining walls $\phi_{\text {wall }}$ is less than in the bulk of the material $\phi_{\text {bulk }}$ as the walls frustrate efficient packing.

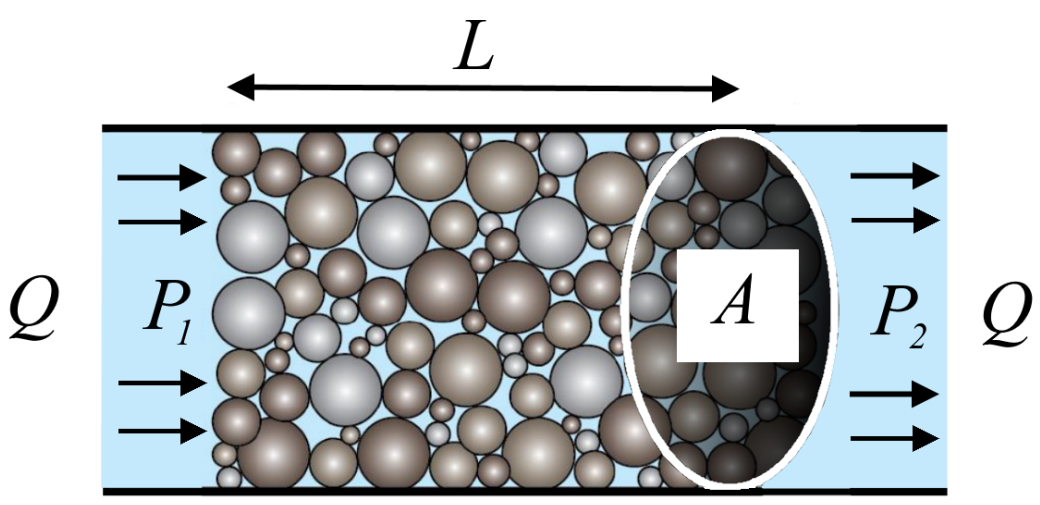

Figure 2.2: Diagram illustrating Darcy flow. A fluid with a flow rate $Q$ passes through a porous material of length $L$ and cross-sectional area $A$, while being driven by pressure $P_{1}$ before the material and experiencing pressure $P_{2}$ after it.

This simple geometrical model predicts a linear decrease of $\phi$ with the "confinement number" $d / b$ which has been found to adequately describe experimental results for monodisperse spheres [30, 37] and simulations of bidisperse spheres [38]. Polydispersity and particle angularity have also been found to have a linearly decreasing relationship, though a lesser confinement effect is found compared with monodisperse spheres [39].

\subsubsection{Darcy's Law}

One of the most famous experiments using a porous material was first performed by Henry Darcy in 1856 [40]. By having water flow through a bed of sand and recording the hydraulic head before and after the sand bed he discovered a linear relationship between the 
flow and the pressure difference across the porous media. The pressure required for fluid to flow can be induced by gravity or compression from a piston for example. This linear relationship between flow and pressure is mediated by how much resistance the water experiences while flowing through the porous medium in an analogous way to resistance for flow of electrical charge in Ohm's Law. Through further experimental work, the parameters that determine the resistance to flow for any fluid in a porous media were determined to complete Darcy's law as follows:

$$
Q=-\frac{k A}{\eta L} \Delta P
$$

Where $Q\left(\mathrm{~m}^{3} / \mathrm{s}\right)$ is the volumetric flow rate of the water, $k\left(\mathrm{~m}^{2}\right)$ is the permeability of the porous media, $A\left(\mathrm{~m}^{2}\right)$ is the cross sectional area of the porous media perpendicular to the direction of fluid flow, $L(\mathrm{~m})$ is the length of the porous media, $\eta(\mathrm{Pa} . \mathrm{s})$ is the viscosity of the flowing fluid, $\Delta P(\mathrm{~Pa})$ is the pressure drop across the porous media $\left(P_{2}-P_{1}\right)$, and the negative sign is due to fluids flowing from higher to lower pressures, see Figure 2.2 . Despite its elegance, a mathematical proof for Darcy's Law beginning with the NavierStokes equation was not derived for over 100 years after Darcy's original publication [41, 42].

The majority of the terms in Darcy's law are trivial to control and measure in an laboratory environment, whereas the permeability of a volume of porous medium is more complex to consider. As such, by controlling the other variables, Darcy's law is regularly used to calculate the permeability of a porous medium in many fields of research relating to geological formations.

\subsubsection{Kozeny-Carman Equation}

The permeability of a granular material can also be estimated with its porosity and grain size using the Kozeny-Carman equation. Derived by Josef Kozeny in 1927 and refined by Philip C. Carman in 1937, the equation estimates the permeability of a granular packing using the size of the grains and the porosity [43, 44]:

$$
k=\frac{\Phi^{2} d^{2}}{180} \frac{n^{3}}{(1-n)^{2}} .
$$


Where $\Phi$ is the sphericity of the particles in the packing ( $\Phi=1$ for spheres), and $d(\mathrm{~m})$ is the diameter of the volume equivalent spherical particle. This equation is applicable for creeping flows (i.e. low Reynolds number) and is derived by modelling the granular packing, with tortuous passages of pore spaces of varying cross sectional areas, as a number of parallel narrow tubes that fill the area of the packing through which Poiseuille flow occurs. The permeability is related to the packing porosity and the surface area that the fluid is in contact with, which can be geometrically estimated using the median particle diameter.

A result of this relationship is that for two sets of spheres with different diameters packed with the same porosity, the spheres with the larger diameter will have a significantly larger permeability as $k \propto d^{2}$. Smaller grains have a lower permeability because of the increase in surface area that the fluid is in contact with.

\subsubsection{Surface Tension}

Liquid molecules have a weak self cohesion that differentiates them from the strongly cohesive solid phase and the non-cohesive gaseous phase. This weak cohesion exerts a zero net force on molecules in the body of a liquid, however a surface molecule experiences a force pulling it into the liquid. This is surface tension, where the surface of a liquid acts as a membrane experiencing tension. Surface tension causes a liquid to take shape with the least surface area possible, requiring a force to expand it. Mathematically, surface tension is defined as the amount of force required to increase the length of liquid surface by a unit length $L$ [45]:

$$
\gamma=\frac{F}{L}
$$

Where $\gamma$ is surface tension $\left(\mathrm{Nm}^{-1}\right)$, and $F$ is force $(\mathrm{N})$. Water has a surface tension of $7.2 \times 10^{-3} \mathrm{Nm}^{-1}$ at $25 \mathrm{C}^{\circ}$.

\subsubsection{Young-Laplace Equation}

A bubble of air, submerged in water, is shaped into a sphere by the surface tension of the water minimising its surface area. The size of the sphere will depend on the pressure inside the air $\left(P_{i}\right)$ balancing against the pressures of the water. The pressure the water exerts on the air bubble is a combination of the pressure of the water outside the bubble $\left(P_{o}\right)$ as well as the pressure exerted by its surface tension on the bubble $\left(P_{S}\right)$. Therefore, the pressure in the air inside of a bubble is always larger than the pressure in the water outside of it:

$$
P_{i}=P_{o}+P_{s}
$$

The surface tension pressure can be calculated with Equation 2.5 by considering one hemisphere of the bubble affixed to the other. In this case $\gamma . L$, which is the force of the surface tension acting to keep the circumference of one hemisphere affixed to the other, 


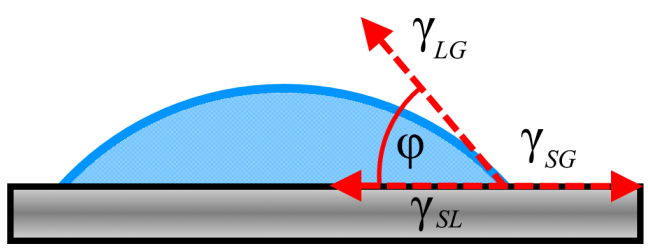

a)

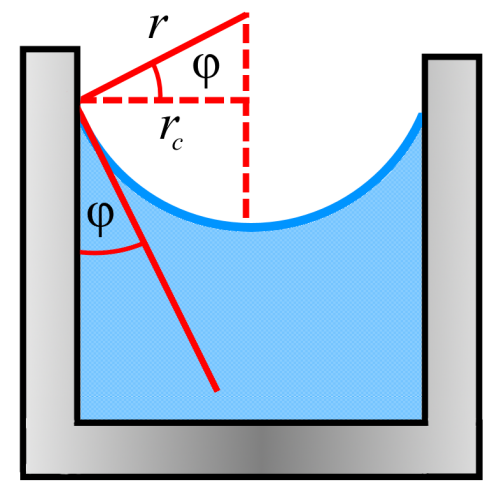

b)

Figure 2.3: Illustration of gas-liquid-solid interface contact angles. a) A fluid rests on top of a surface creating a contact angle $\phi$ that depends on the solid-liquid, liquid-gas, and solid-gas surface tensions, $\gamma_{S G}, \gamma_{L G}$, and $\gamma_{S G}$ respectively. b) A fluid rests in a container with a radius $r_{c}$ where surface tension pulls the fluid up the container walls with a contact angle $\varphi$ forming an interface with a radius of curvature $r$.

is equivalent to $F$, the force of the internal pressure acting over the cross-sectional area of the bubble:

$$
\gamma \cdot 2 \pi r=P_{s} \pi r^{2}
$$

where $r$ is the radius of the bubble. Cancelling terms, this equation can be rearranged to find the surface tension and pressures across a spherical interface:

$$
P_{s}=P_{i}-P_{o}=\frac{2 \gamma}{r}
$$

or $P_{s}=\gamma\left(\frac{1}{r_{1}}+\frac{1}{r_{2}}\right)$ for surfaces with two radii of curvature. This simplified relationship, equating the pressure caused by surface tension trying to minimise a curved surface, with the difference between the pressure inside and outside of that surface, is called the YoungLaplace equation after it was developed by Thomas Young and formally derived by PierreSimon Laplace in 1805 [46, 47].

\subsubsection{Contact Angle}

When a liquid-gas interface comes into contact with a solid material, surface properties with cohesive and adhesive forces determine a triple contact line, see Figure 2.3 a). The cohesive forces in the liquid act to pull it away from the solid surface while adhesive forces on the solid surface act to spread the liquid across the solid. The equilibrium point 
between the three surface tensions is characterised by a contact angle $\theta$ :

$$
\gamma_{S G}-\gamma_{S L}-\gamma_{L G} \cdot \cos (\theta)=0
$$

Where $\gamma_{S G}, \gamma_{S L}$, and $\gamma_{L G}$ are the surface tensions between the solid-gas, solid-liquid, and liquid-gas phases respectfully. For $\theta<90^{\circ}$, the liquid wets the surface of the solid, while for $\theta>90^{\circ}$ the gas wets the solid. Materials with high surface energies tend to wet liquids because of their particularly strong cohesive bonds, for example metals, glasses, and ceramics. Materials with relatively low surface energies tend to be wet by gasses because of their particularly weak cohesive bonds, for example plastics, waxes, and composites. Imbibition occurs when a fluid that wets a porous medium displaces a defending nonwetting fluid, where as drainage occurs when a non-wetting fluid displaces a wetting fluid within a porous medium. The experiments performed in this thesis deal with drainage.

Using the triple line contact angle, the Young-Laplace equation can be modified for a liquid-gas interface between a solid confinement i.e. a tube or pore space.

$$
P_{s}=P_{i}-P_{o}=\frac{2 \gamma \cos (\theta)}{r_{c}}
$$

Where $r_{c}$ is the radius of the pore space, see Figure $2.3 \mathrm{~b}$ ). In the context of this thesis it is important to note that smaller grains have smaller pore spaces; the smaller a pore space the larger the pressure is across the liquid-gas interface.

\subsubsection{Friction and the Janssen Effect}

So far only a static porous medium has been considered but in the systems studied here the granular packing is deformable: grains will move around each other and against the confining Hele-Shaw cell. Friction is the force that resists motion between two surfaces in contact. The maximum frictional force between surfaces $\left(F_{f}\right)$ is proportional to the normal contact force $(N)$ and the surface contact's coefficient of friction $(\mu)$ :

$$
F_{f}=\mu N
$$

As long as the force exerted to move the surfaces is below this maximal value of frictional force, the frictional force will equal the moving force and so the surfaces will not move. Once the moving force exceeds this value motion begins and is then subject to a constant kinetic friction, assuming nothing else changes.

A very simple but well used estimate for the coefficient of friction for a granular material uses the angle a pile of it makes with a surface when it is poured onto it, called the angle of repose:

$$
\tan (\theta) \approx \mu
$$


The angle of repose helps explain the regular angles observed in sand dunes, see Figure 1.2. In a granular system friction is present in every grain-grain contact as well as between each grain that is in contact with a wall of the container.

When a container is filled solely with a liquid, the pressure on the area at the bottom of the container is equal to the weight of the liquid above it:

$$
P=\rho g h
$$

Where $P$ is the pressure, $\rho$ is the liquid's density, $g$ is the acceleration from gravity, and $h$ is the height of the water above the area where the pressure is measured. Intuitively, if you add more water to the system the pressure experienced at the bottom of it proportionally increases [45].

However, granular material does not behave this way. Grains disperse pressure exerted on them to the walls of the container via frictional grain-grain contacts, called force chains. In 1895, H. A. Janssen determined that the horizontally redirected pressure experienced by the walls of the container $\left(P_{r}\right)$ in a granular packing is approximately proportional to the vertical gravitational pressure $\left(P_{d}\right)$ at that given height. This proportionality is governed by a material-specific property now called the Janssen coefficient $(\kappa)$ [48]:

$$
P_{r}=\kappa P_{d}
$$

These force chains form arches in the granular material, becoming supported between the walls of the container with friction while exerting less pressure on the grains beneath them. This means that adding more grains to a container does not directly increase the pressure at the bottom of it, but instead has diminishing effect as more and more of the weight is supported by the walls of the container. This diminishment lasts until a threshold of grains have been added to the packing, after which the pressure at the bottom of the packing no longer increases as grains are added, instead the normal force with the container's walls increases as it holds up the packing.

The Janssen effect is also relevant to granular material that is being driven by a nongravity force in a horizontal container, such as grains being forced to move through a Hele-Shaw cell. Knudsen et al. and Marks et al. derived the pressure, $P_{F}$ required to displace a compacted granular packing of length $L$ between two horizontal plates separated by distance $b$ which is reproduced here, see Figure 2.4 [22, 49].

Assume a Janssen relation that states that the out-of-plane stress $\sigma_{z z}$ caused by the the driven in-plane stress $P_{F}=\sigma_{x x}$ is proportional by $\sigma_{z z}=\kappa \sigma_{x x}$, where $\kappa$ is the Janssen coefficient. The force exerted by the driving pressure in the $X$ direction on the $\Delta Y \Delta Z$ front of the granular packing at point $X$ required to move the front up to a point a small distance away $(X+\delta x)$, is equal to the reactive force at that point, the normal force from 


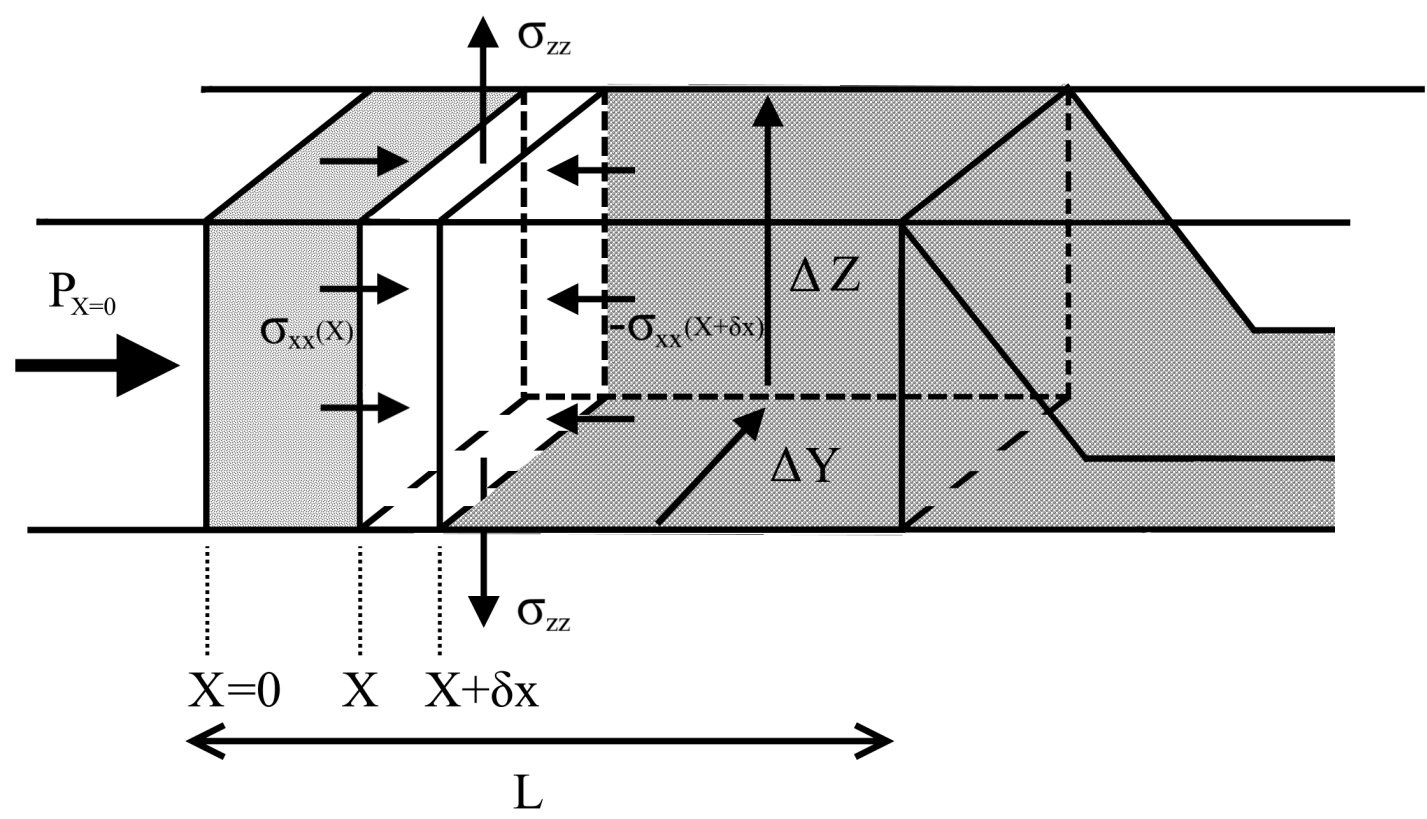

Figure 2.4: Illustration of the Janssen effect for a granular packing in a Hele-Shaw cell. A compacted region of granular material of length $L$ and width $\Delta Y$ in a confined space of height $\Delta Z$ is subject to a driving pressure $P$ at its open interface $X=0$. Where $\sigma_{x x}$ is the stress experienced by the packing from the driving pressure and $\sigma_{z z}$ is the stress experienced by the packing from the the driving pressure being redirected as friction against the confining plates according to the Janssen effect. At $X=L$, beyond the compacted region, the packing transitions to an uncompacted region.

the weight of the packing, as well as the frictional force exerted on the two $d x d y$ areas of the granular packing contacting the cell plates above and below it in the $Z$ direction:

$$
\left(\sigma_{x x}(X)-\sigma_{x x}(X+\delta x)\right) \Delta Y \Delta Z-g \rho_{e} \delta x \Delta Y \Delta Z \mu-\mu \sigma_{z z}(X) 2 \delta x \Delta Y=0
$$

Simplifying by assuming the gravitational term is insignificant to the frictional forces in play, using the Janssen relation outlined, dividing each term by $\delta x \Delta Y \Delta Z$, given that $\sigma_{x x}(X)-\sigma_{x x}(X+\delta x)$ is $\sigma_{x x}(\delta x)$, and that the distance $\Delta Z$ is equivalent to the distance between the plates, $b$, a differential equation is obtained:

$$
\frac{\delta \sigma_{x x}(x)}{\delta x}=-\frac{2 \mu \kappa \sigma_{x x}(x)}{b}
$$

This is then integrated over $L$ to find the pressure required to displace a packing of that length. To do so it is taken that the pressure at the boundary $L$, in the uncompacted granular packing not experiencing the driving pressure, is due to the granular mass averaged over the cell gap:

$$
P_{T}=\frac{1}{2} b \rho_{e}\left(1-n_{0}\right) g
$$


where $\rho_{e}$ is the effective density of granular material in the interstitial fluid, $n_{0}$ is the uncompacted porosity, and $g$ acceleration due to gravity. Using this boundary condition, Equation 2.16 integrates as:

$$
P_{F}=P_{T} \exp \left(2 \mu \kappa \frac{L}{b}\right) .
$$

Therefore the pressure required to displace a compacted granular interface in a planar geometry increases exponentially with the length of the compacted region of the packing.

\subsection{Suspended Granular Physics}

A suspension is a liquid that contains solid particles that have not settled, do not dissolve, and are not small enough to be dominated by Brownian motion. As a result of these characteristics, the solid particles flow with the interstitial liquid; the combined solidliquid system can be treated as a continuous fluid that has rheological properties like viscosity.

\subsubsection{Viscosity}

The viscosity of a fluid is a measure of how much it resists deformation and is very much analogous to friction. It can be defined simply using Couette flow, the flow induced in a fluid between two parallel surfaces while the top surface moves relative to the bottom [45], see Figure 2.5.

Assuming a no-slip boundary (the fluid touching the bottom plate is not moving while the fluid touching the top plate moves with its velocity) the stress required at the top plate to move the fluid with velocity $u$ is proportional to the gradient of the velocity across the separation of the plates $y$, where the proportionality term is the fluid's resistance to that motion:

$$
\sigma=\eta \dot{\gamma}
$$

Where $\sigma(\mathrm{Pa})$ is shear stress, $\eta$ (Pa.s) is viscosity, and $\dot{\gamma}=\frac{d u}{d y}\left(\mathrm{~s}^{-1}\right)$ is the shear rate. A fluid's viscosity is a property that is isothermally constant for a Newtonian fluid such as water.

Couette flow is used to study the viscosity of a liquid with a rheometer, where a surface with a known geometry rotates against a liquid between a stationary surface. The geometry of the rotating surface, its height above the stationary surface, the speed that it rotates, and the force that is required to rotate it are used to calculate the fluid viscosity:

$$
\eta=\frac{F / A}{\Delta h / \Delta v}
$$




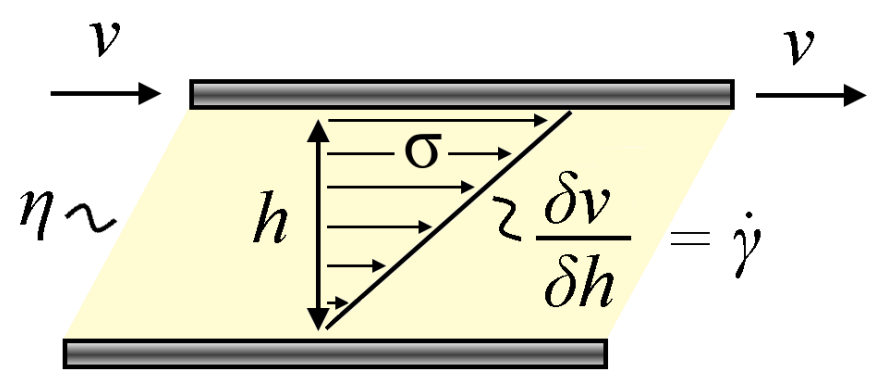

Figure 2.5: Diagram of a simple parallel shear flow used to determine fluid viscosity. Fluid experiences a shear stress $\sigma$ required to move the top plate at a velocity $\eta$. It experiences no stress at the bottom stationary plate. The gradient of the velocity over the distance between the two plates $\frac{\delta \eta}{\delta h}$ is equivalent to the shear rate $\dot{\gamma}$. The viscosity of the fluid is measured as the ratio between the shear stress and the shear rate.

where $F$ is the force imposed by the moving surface, $A$ is the area of the plate, $h$ is the plate gap, and $v$ is the velocity of the plate. There are three primary rotational geometric setups used in rheometry, see Figure 2.6, with "parallel-plate" being the simplest. In parallel-plate rheometry a plate rotates above a parallel stationary plate. In "cone-andplate" geometry a shallow angled conical plate rotates with its centre almost touching the stationary bottom plate. And in "Couette" geometry the liquid is held between two vertically aligned cocentric cylinders while the smaller inner cylinder rotates, with the difference between the radii of the cylinders being the gap size.

\subsubsection{Viscosity of a Suspension}

It has been known for over a hundred years that gradually adding hard spheres to a fluid to form a suspension will increase its viscosity. A model for viscosity as a function of

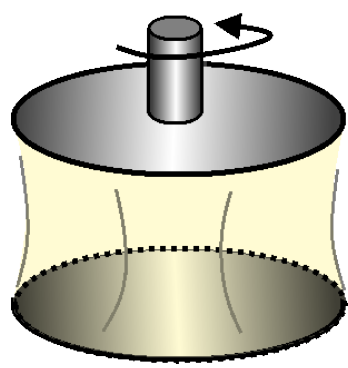

a)

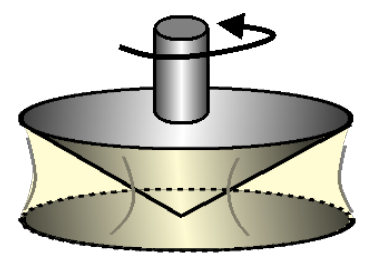

b)

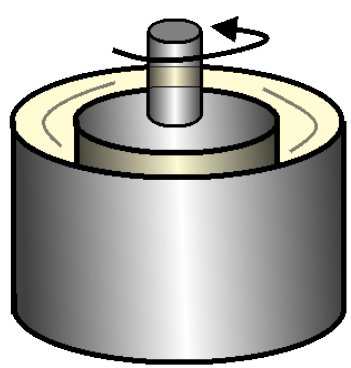

c)

Figure 2.6: An example of three rheometry geometries. a) Fluid is sheared between two parallel discs, the higher of which rotates. b) Fluid is sheared between a disc and a parallel rotating cone. c) Fluid is sheared between two concentric cylinders, the smaller of which rotates. 


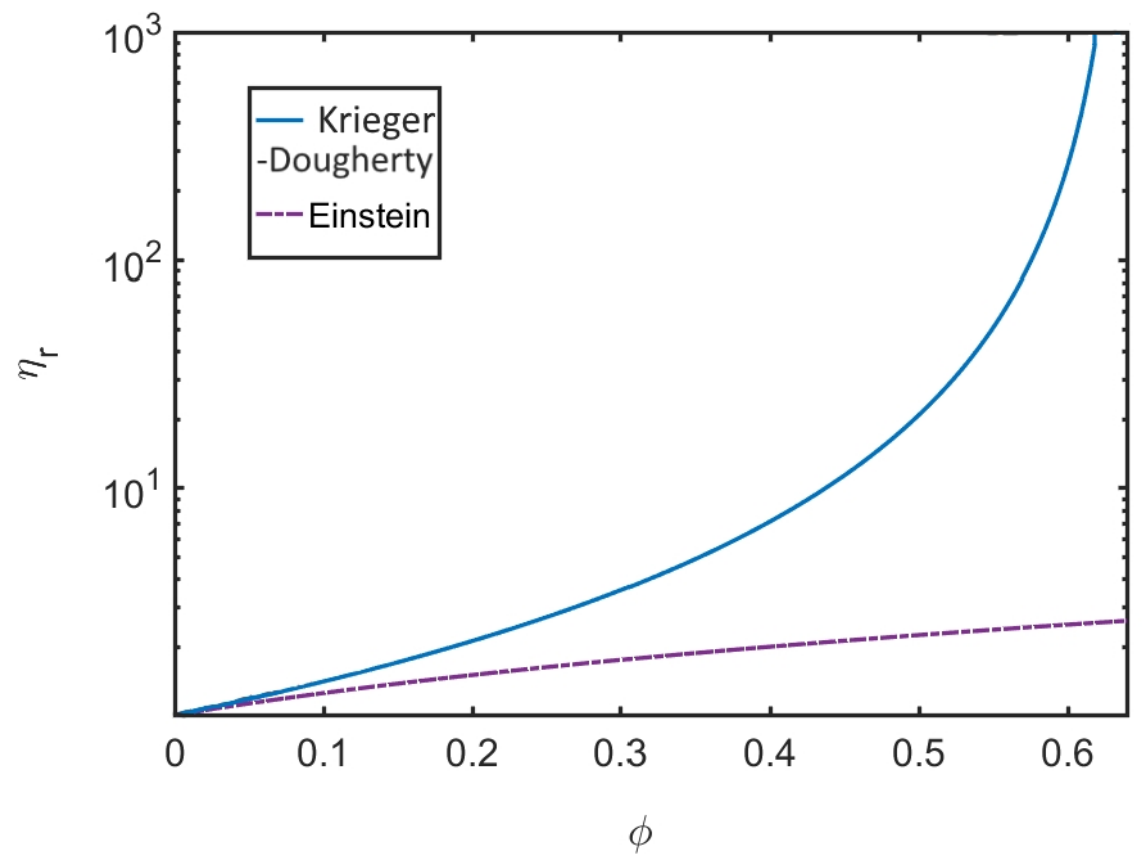

Figure 2.7: Graph illustrating the viscous jamming of a granular suspension as its volume fraction approaches $\phi_{c}$ modelled by the Krieger-Dougherty equation as well as the dilute limits of Einstein's initial model.

volume fraction of non-interacting spheres was first theoretically proposed by Einstein, see Figure 2.7 [50, 51].

$$
\eta_{r}=\frac{\eta}{\eta_{0}}=1+2.5 \phi
$$

where $\eta_{0}$ is the viscosity of the interstitial fluid, $\eta$ is the viscosity of the suspension, $\eta_{r}$ is the reduced viscosity, and $\phi$ is the volume fraction of the granular material. However, this linear model only applies to dilute suspensions.

As the suspension approaches the random close packing (RCP) limit for spheres the viscosity begins to drastically increase until the system jams. Models that replicate this jamming at RCP with frictionless hard-spheres model take the established form of:

$$
\eta_{r}=\left(1-\frac{\phi}{\phi_{0}}\right)^{-\beta}
$$

Where $\phi_{0}$ is the RCP volume fraction and $\beta$ is a constant that ranges from 1.5-2 [52-54]. This model is often referred to as the Krieger-Dougherty model. 


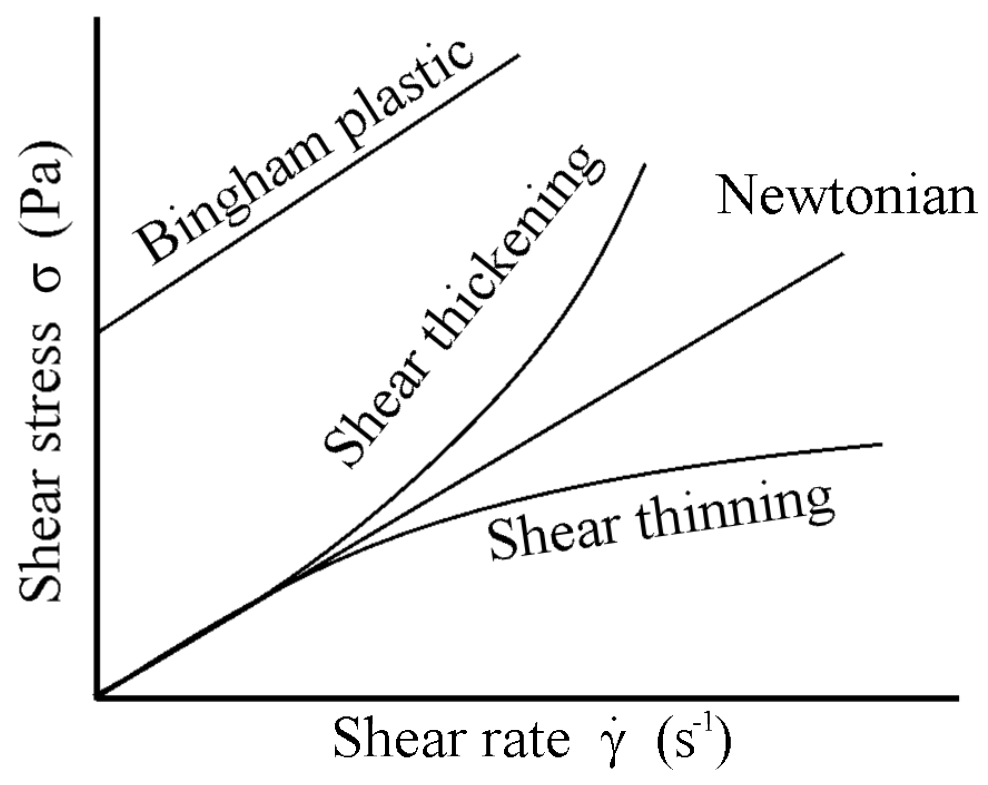

Figure 2.8: Diagram illustrating different archetypal non-Newtonian fluid curves in contrast to a Newtonian fluid that has a constant gradient that passes through the origin.

\subsubsection{Non-Newtonian Fluids}

Adding particles to a Newtonian fluid does not just increase it viscosity, it also introduces other complex interactions and forces to the system that cause its viscosity to not be a constant property but instead a variable that depends on imposed deformations. This varying behaviour can be illustrated on a rheological flow curve, where the viscosity is the gradient of the shear stress versus shear rate curve, see Figure 2.8 .

A Newtonian fluid has a linear gradient on its flow curve. For non-Newtonian fluids there are primarily three models used to describe behaviour as a function of an imposed shear. A fluid for which its viscosity is constant with shear but has a yield stress (will not flow until an imposed stress limit is reached) is called a Bingham plastic or yield-stress fluid, examples of which include toothpaste, mayonnaise, and mud. A fluid for which its viscosity decreases with increasing shear rate is a shear thinning fluid, examples of which include ketchup, paint, and blood. Lastly, a fluid for which its viscosity increases with an increasing shear rate is a shear thickening fluid, examples of which include cornstarch suspensions, some magma suspensions, and some polymer suspensions. In extreme cases, small increases in the shear rate increase the viscosity of the suspension orders of magnitude, resulting in a discontinuous transition to a considerably more viscous fluid. This is known as Discontinuous Shear Thickening (DST).

A generalised model for these three behaviours is the Herschel-Bulkley fluid, which was developed by Winslow Herschel and Ronald Bulkley in 1926 [55].

$$
\sigma=\sigma_{0}+k_{i} \dot{\gamma}^{n_{i}}
$$


where $\sigma_{0}(\mathrm{~Pa})$ is the yield stress of the fluid, $k_{i}$ is the consistency index of the fluid, and $n_{i}$ is the flow index of the fluid; where $n=1$ for a Newtonian fluid, $n<1$ for shear thinning, and $n>1$ for a shear thickening fluid.

However, this model only applies to more idealised non-Newtonian fluids, and is commonly used to describe concrete, dough, and mud for example. It has not been particularly useful in literature on cornstarch suspensions. 


\section{Chapter 3}

\section{Literature Review}

Multiphase flows in deformable porous media are particularly complicated to study and model because no phase in the system is static: there is a moving boundary between the two competing fluids and this fluid flow can also mobilise the solid material surrounding them. As the porous media can change over time, additional processes need to be considered when compared to systems with fixed or no solid matter. Compaction, segregation, and jamming can decrease the permeability of a system, whereas decompaction, fracturing, and fluidisation can increase the permeability of a system. Compaction and jamming events can also spontaneously transform regions of previously deformable solid media into non-deformable media during a flow process. A dynamic feedback is thus established as the fluid flow alters the characteristics of the porous medium whilst these changes then alter the characteristics of the flow.

Another significant complication for the study and modelling of multiphase flows in deformable media is that processes at different sizes and time scales influence each other and all contribute to observed behaviour. Individual inter-pore scale events that depend on a subtle interplay between local fluid pressures, wettability, and pore sizes on the micrometer and micro-second scales influence flow behaviours and inter-granular motion that depend on granular shape, friction, and immediate viscous forces on the millimeter and second scales, which in turn influence system-wide flow patterns that depend on macroscopic properties like permeability, compactibility, and pressure gradients that can span geological systems for miles and eons. 
As a result of the complicated interplay between different phases, materials, physics, and time-scales, multiphase deformable flow is of interest to many different fields of study, including granular physics, flow dynamics, system jamming, geo-physics, soft matter, rheology, and complex fluids. This chapter will discuss practical studies in these fields that are of direct relevance to this thesis, what work has already been done in these fields that has explored the immediate space around this thesis, as well as the related questions that remain in these fields, some of which this thesis aims to answer. This literature review will be divided into two sections, one on systems where the deformable porous media has settled under gravity in the interstitial fluid and the other on systems where the deformable porous media is made up of granular material that is suspended in the interstitial fluid.

\subsection{Fracturing in Settled Granular Media}

Two examples of work that has real world applications for which it is crucial to have a further understanding of multiphase flows in settled granular media will be discussed here. The first is the industrial process of carbon dioxide capture and storage and the second is the natural process of methane hydrate venting from the sea floor; both of which are intimately linked with complex gas migration behaviour in porous networks.

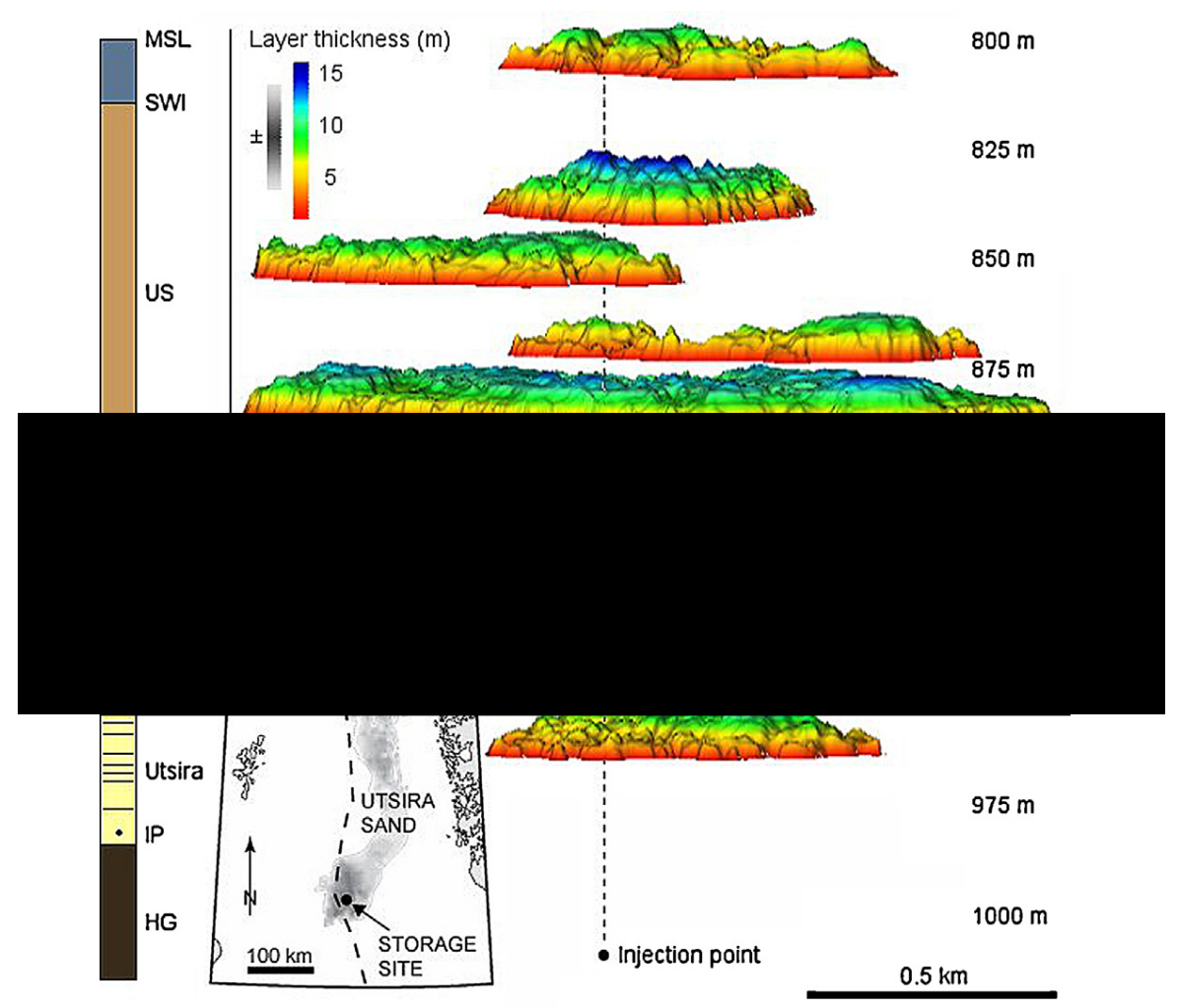

Figure 3.1: Data of $\mathrm{CO}_{2}$ plume progression through Sleipner storage aquifer from July 2002, Cavanagh et al. (2014) [12]. The left panel is a regional stratigraphic column: MSL, Mean Sea Level; SWI, Sediment Water Interface; The Nordland Group extends from the caprock to seafloor, and is subdivided into three seals: US, Upper Seal; MS, Middle Seal; LS, Lower Seal; Utsira, storage site; HG, Hordland Group. 


\subsubsection{Carbon Capture Storage}

Carbon Capture Storage (CSS) has been claimed by the G8 and International Energy Agency to be a necessary technology for mitigating greenhouse gas emissions and preventing ocean acidification [12]. CCS is a process by which the $\mathrm{CO}_{2}$ emitted by a power station or gas field is captured, compressed, and injected via pipeline into porous media formations underground and undersea. This injection will displace a defending liquid, usually brine, through both rigid and deformable porous media and is prevented from escaping to atmosphere by a natural non-porous caprock or seal. For example, the world's largest and first attempt at CSS is the Sleipner storage site off the coast of Norway. Each year since 1996 one mega ton of $\mathrm{CO}_{2}$ produced in a gas field is injected $1 \mathrm{~km}$ under the sea bed into a saline aquifer. A 100 m thick stack of shale layers that sits between the storage site and the seafloor is the intended seal with a high enough threshold pressure to prevent venting and release for thousands of years [56]. However, concerns for leakage are apparent.

Seismic surveys, gravimetric measurements, and electromagnetic monitoring have found that the $\mathrm{CO}_{2}$ has made its way vertically through several layers of shale much faster than expected and reached the top seal in less than 3 years, see Figure 3.1 [57, 58]. Multiphase flow modelling has suggested that the shale barriers have a surprisingly low threshold pressure and imply that the quick vertical transport of the $\mathrm{CO}_{2}$ plume has been enabled by fracture networks in the shale [12]. However, the same modelling has shown that the pressure in the injected $\mathrm{CO}_{2}$ is not enough to have caused the fracturing, instead it is believed that the shale had fractures before the site was used for CSS [12]. This kind of significant defect was not detected when the site was first investigated for CSS but also was not being looked for. Stresses from the deglaciation of ice sheets over tens of thousands of years go is the suspected cause of the fractures and it has been suggested that other shallow North Sea storage sites could have similar defects that should be considered before use. 


\subsubsection{Methane Venting}

Natural venting of methane hydrate through seafloor sediments represents a significant source of greenhouse gas emission to the atmosphere [60]. An example of which is the East Siberian Arctic Shelf, a submersed extension of the Siberian tundra $200 \mathrm{~km}^{2}$ in size, that is being studied for its venting. Here the sea bed is made of frozen sediments formed from flooding that occurred over 7 thousand years ago [61]. This flooded peatland contains deposits of methane hydrate that are sealed by sub-sea permafrost [62]. Expeditions are being performed to investigate the venting occurring here as it can contribute to a positive feedback loop with regards to climate change [60]: as the climate warms from the greenhouse gas effect, more thawing will occur which will increase the sub-sea permeability and so more methane will be able to flow out to the climate accelerating the greenhouse effect in turn [63, 64].

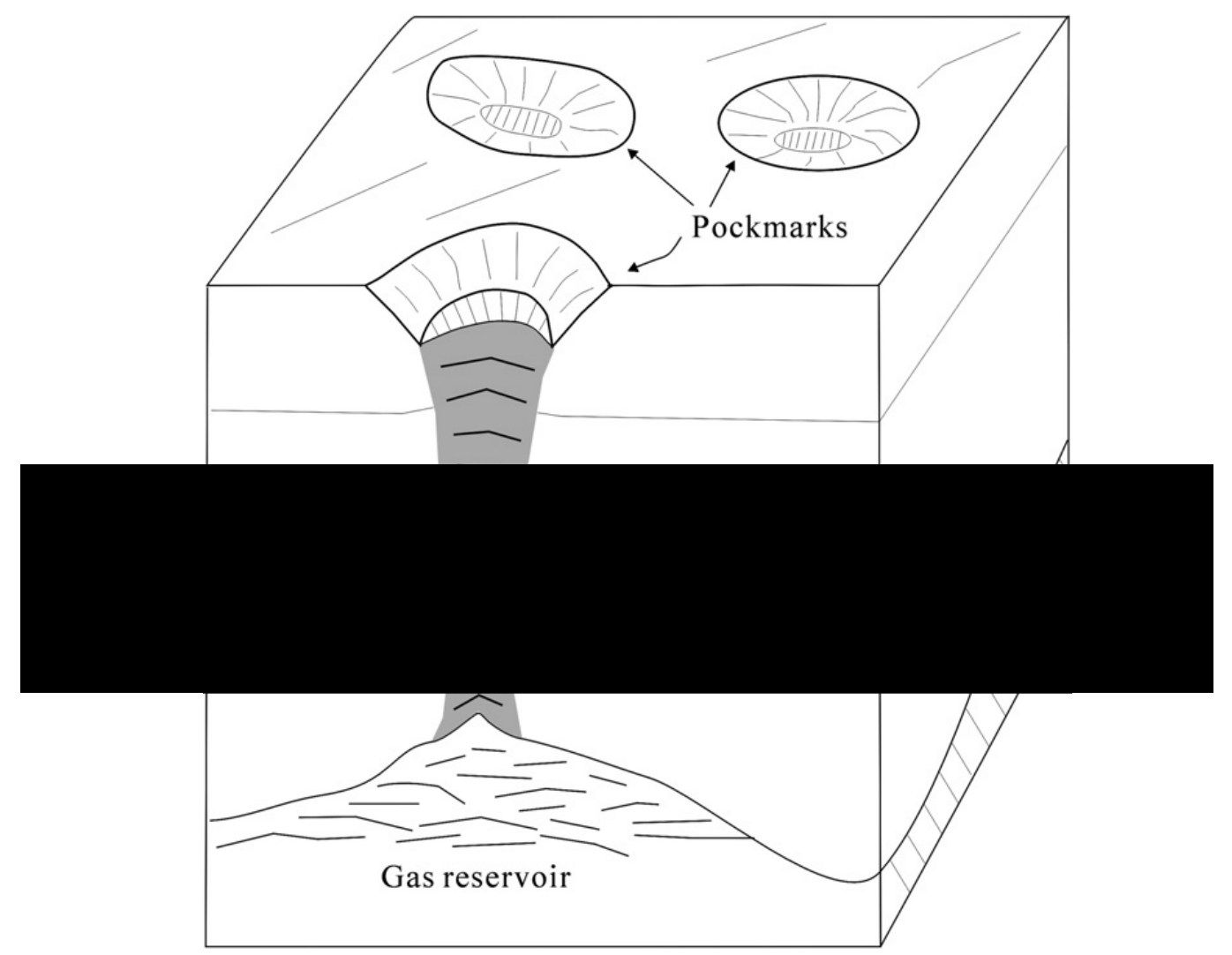

Figure 3.2: Diagram of subsurface features that form pockmarks from Cathles et al. (2010) [59]. 
Pockmarks are an example of a natural seafloor venting process. Pockmarks are a crater-like depression found on granular seafloors; discovered in the 1970s, pockmarks have since been observed with sonar in oceans all over the world as well as in some lakes [65]. Pockmark formation requires a layer of fine-grained sediment that acts as a capillary seal, preventing gas from rising through it due to the narrow pore spaces. However the capillary spaces can be invaded when a critical pressure beneath the seal is reached as enough gas accumulates from below. When the seal fails, the large deposit of gas rushes up through the porous media, displacing the water and mobilising the granular bed to form a "chimney". As the chimney ascends towards the seafloor surface, the mobilised grains are carried away by ocean currents and so the distinctive pockmark-crater is formed once this multiphase flow process is complete, see Figure 3.2 [59]. As a result, wherever pockmarks are observed there is often trapped gas deposits below the seafloor that is likely to intermittently vent as subsurface pressures are built up and then released [65].

\subsubsection{D model systems}

The processes of gas transport through saturated deformable sediments via bubbles and fracturing are not understood well enough to establish an accepted rheological flow model [10]. To help elucidate the mechanisms involved, many experiments have been performed to model the processes. A clear analogue that has been used extensively is that of a column of granular material (usually glass beads) submerged within a fluid inside a cylindrical container, at the bottom of which a gas is injected while the top surface is examined for bubbles, see Figure 3.3 [9. 11, 66, 67].

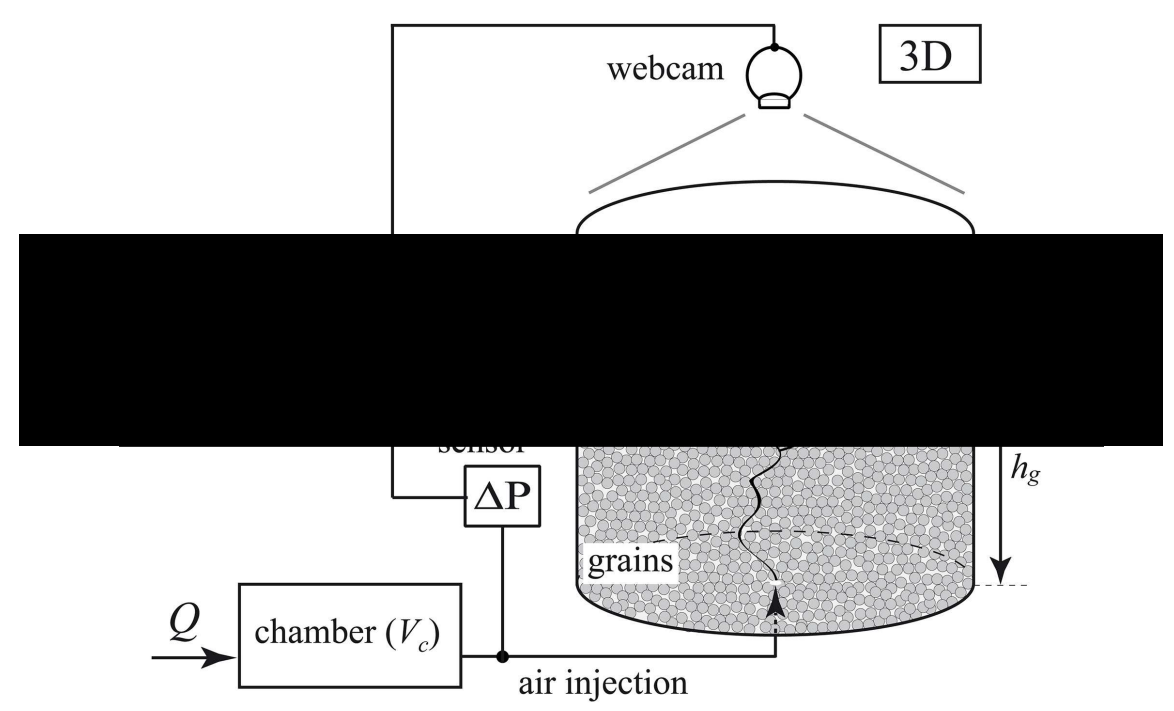

Figure 3.3: Setup for 3D experiment of gas rising through a submerged granular material from Varas et al. (2015) [67]. 
Different flow regimes have been reported in these systems depending on a variety of parameters. Altering the size of the grains changes the capillary entry pressure between pore spaces. Varas et al. (2011) [11] found that for "large" grains $(425-600 \mu \mathrm{m})$ the entry pressure is low enough that the injected air passes freely through the granular column and bubbles emerge at the surface with high frequency. For "small" grains $(150-250 \mu \mathrm{m})$ the capillary entry pressure is high enough that the injected air does not immediately pass through the granular material. Instead it collects at the inlet into a larger bubble that deforms the general packing such that a "dome" can be observed at the surface until the accumulated pressure in the injected air is either high enough to overcome the weight of the granular material and rise to the surface or burst through the capillary pressure between the grains. For "intermediate" grains $(250-425 \mu \mathrm{m})$ bubbles were observed at the surface intermittently but without observable large-scale deformation of the granular packing [11].

In the intermediate intermittent regime, a diffusion-like process determines the spatial distribution of the surfacing bubbles [11]. As a result, the size of the area in which the bubbles surface is related to the depth of the bed as well as the size of the granular material: with smaller grains having a wider spread and larger grains having a narrower one [11]. If the gas injection rate is increased significantly constant bubbling is observed, likely caused by channels (though unobserved) being forced open in the granular packing to accommodate the more rapid gas invasion. In middling flow rates the system dynamically alternates between the intermittent and channeling regimes [67].

Dalbe and Juanes (2018) [66] performed this experiment with both a transparent interstitial fluid and transparent beads with a contrast between them and the invading fluid. Using image thresholding, based on the brightness of the depth of field through the column, they produced a 3D image of fluid and granular disturbance. They confirmed a transition from capillary invasion (the granular media is not deformed) to the "opening of conduits" (granular media is deformed) as a balance between capillary and viscous forces against confinement and frictional forces [66]. Sun and Santamarina (2019) [68] reported similar findings, stating that "Gas migration is grain-displacive at low effective stress $\left(\sigma^{\prime}\right)$ and high air entry pressure $\left(\Delta P_{A E}\right)$ so that $\Delta P_{A E} / \sigma^{\prime} \gg 1$, and becomes pore-invasive when $\Delta P_{A E} / \sigma^{\prime} \ll 1$ ". Fracturing is reported at higher effective stresses within the grainsdisplacive regime, with growth occurring perpendicular to the minimum axis of stress in the packing; therefore fractures were vertical in typical packings but stresses imposed on the packing before experimentation could rotate the direction of fracture growth [68]. 


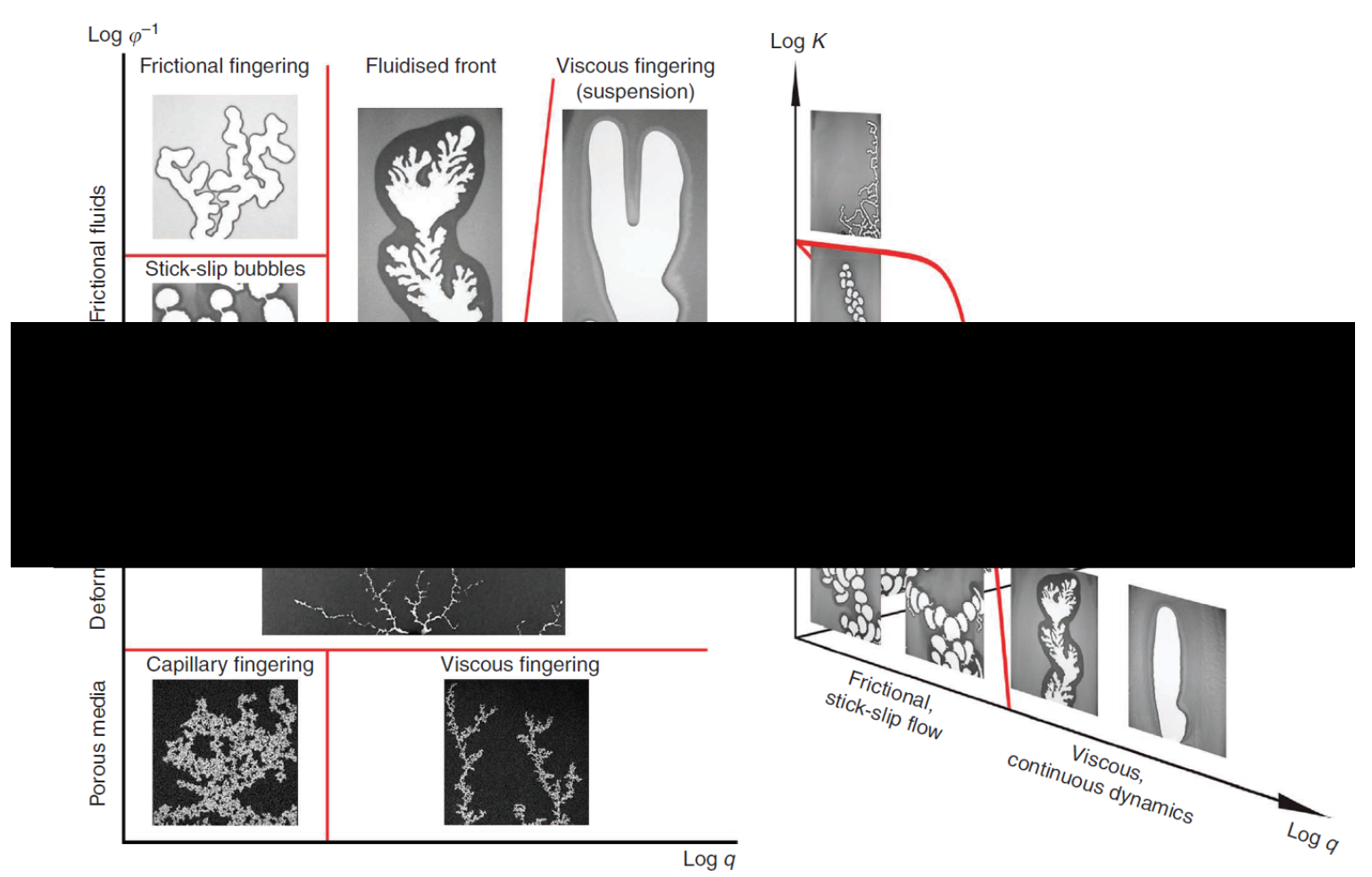

Figure 3.4: Phase diagram of granular Hele-Shaw cell invasion patterns by Sandnes et al. [21]. The phases are roughly classified in relation to $q$ the injection rate of the invading air, $\phi^{-1}$ the inverse of the ratio of the cell occupied by grains, and $K$ the system stiffness.

Like the real world examples they are emulating, these experiments are incredibly complex. Buoyancy, capillary, viscous, and frictional forces all need to be considered to model the different flow behaviours. In addition, the grains have an open upper boundary, and so can be pushed out of the containing fluid system by ascending gas or fluid motion [67, 68]. Also, the effect of gravity on the mobile grains can cause undesirable sedimentation. A similar setup has even been used with real soil samples (instead of glass beads) that are subject to an invasion of methane, so as to study how the methane dissolving in the water as it rises introduces other complications to analysis [69]. Lastly, efficient imaging of 3D systems for computational processing is limited, making it difficult to quantify how the invading gas displaces the granular media internally, as opposed to just on the surface. To precisely image deformation of the granular media as the gas makes its way through the system the pseudo-2D geometry of a Hele-Shaw cell is used. 


\subsubsection{Three-phase Hele-Shaw Experiments}

Multiphase flow experiments with a deformable porous system conducted with a HeleShaw cell allow a clear visualisation of the invading gas transport and the disturbance it causes in the granular media. A well used setup for these experiments is to have grains submerged in a fluid between two transparent plates separated by a height that allows for the grains to form a packing (as opposed to a mono-layer). The cell can be laid down so that the gas will flow through the cell perpendicular to gravitational effects, so as to minimise the complications of the grains resettling during invasion events or it can be angled to include such phenomenon. Air is then forced into the system and its invasion front is filmed until it reaches an open boundary at an edge of the cell.

Many parameters have been explored in this experimental setup, including the filling fraction of the grains [21, 70, 71], the size of the grains [70], the injection rate of the gas [21, 24], the stiffness of the fluids [21, 23], the introduction of gravitational effects [72], the height of the cell spacing [24, 70], and the confinement pressure [24]. The wettability of the porous medium and the cell has also been studied, though with a static, non-deformable porous medium [73-75].

Focusing here on the resultant patterns, Chevalier et al. (2007) [70] studied the destabilisation of viscous fingers formed in this setup using suspended density-matched particles. The cell gap size, the volume fraction of the grains (up to $\phi=0.40$ ), and the size of the grains were parameters. It was found that the presence of grains (as little as $\phi=$ 0.01 ) creates perturbations in the interface that initiate destabilisation of fingers and tipsplitting at far lower finger velocities than without grains (this premature destabilisation had a comparatively small dependency on increasing $\phi$ ). Later, Chevalier et al. (2009) [71] explored multiphase invasion flows with granular volume fractions up to $\phi=0.60$, which was close to their systems maximal packing fraction of $\phi_{R C P}=0.62$. As $\phi$ increases the invasion finger width decreases from sizes comparative to the cell width, to narrow branches of the order of the cell spacing, to sizes close that of the grains themselves when the packings are close to $\phi_{R C P}$. In the last case the grains are "jammed" and do not move at all, the air invades the pore spaces between grains, which is known as capillary fingering. 
Sandnes et al. (2011) [21] performed experiments to map similar changes in behaviour as a function of $\phi$, as well as injection rate and system stiffness $K\left(\mathrm{~Pa} / \mathrm{m}^{3}\right)$, except with settled granular material as opposed to suspended. The effective packing fraction was here determined by how much of the cell height was occupied by settled grains. The stiffness in the system is governed by how much volume is in the reservoir of gas being injected, as the gas is a compressible fluid where as the interstitial water is not. The settled granular media produced additional phenomenon to those observed by Chevalier et al., including bubbles and frictional fingering, which can be seen in Figure 3.4. For volume fractions in which the cell was not completely full (when full capillary invasion was observed), low injection rates are friction dominated as the system has enough time for particle-particle contacts to be made. The gas invasion then proceeds in an intermittent stick-slip fashion that is characteristic of a frictional system, resulting in a random-walklike pattern for the fractures $\left(\phi_{R L P}\right)$, bubbles (less than $\phi_{R L P}$ ), or fingers (much less than $\left.\phi_{R L P}\right)$ [21]. As the injection rate is increased viscous forces are dominant, with the system becoming fluidised once the rate is enough for the settled grains to be suspended resulting in viscous fingering patterns [21, 71].

\subsubsection{Hele-Shaw Fracturing}

A lot of focus has been directed to describing the transition from flow behaviour to fracturing and from fracturing to capillary invasion in a Hele-Shaw cell [9, 21, 24, 67, 71, 76]. It is now accepted that fracturing occurs when the granular packing fraction is close to $\phi_{R C P}$. Chevalier et. al (2009) [71] described granular fracturing as follows, "when the packing fraction is slightly below jamming, the thin air finger invades the weak porous matrix by breaking and reorganizing the jammed packing of grains". At these high filling fractions the granular system transitions from a frictional fluid to a porous media [21].

Holtzman and Juanes (2012) [24] developed a simple model that describes when a dense granular media will fracture. They studied flow behaviour in a dense three-phase Hele-Shaw system, like those described above, except that the top plate confined the packing with an applied weight $w$, so as to make the confining stress a variable. Grain diameter and air injection rate were also variables. Where previous studies linked the transition in dense packings between fracturing and capillary fingering with $\phi[21,71]$ (maximal packings cannot be deformed so invasion proceeds via capillary fingering), Holtzman and Jaunes re-framed the description with a confining pressure that instills a rigidity in the system that can control the same transitionary behaviour.

Holtzman and Juanes explain how fracturing in dense granular media occurs as follows, "fractures open when forces exerted by the fluids exceed the mechanical forces that resist particle rearrangements." As such, they model the fracturing regime with a fracturing number $N_{f}$ which measures the systems deformability. The $N_{f}$ is a ratio between pressure forces driving fracturing $\Delta f_{p}$ (estimated with the capillary and Darcy pressure 


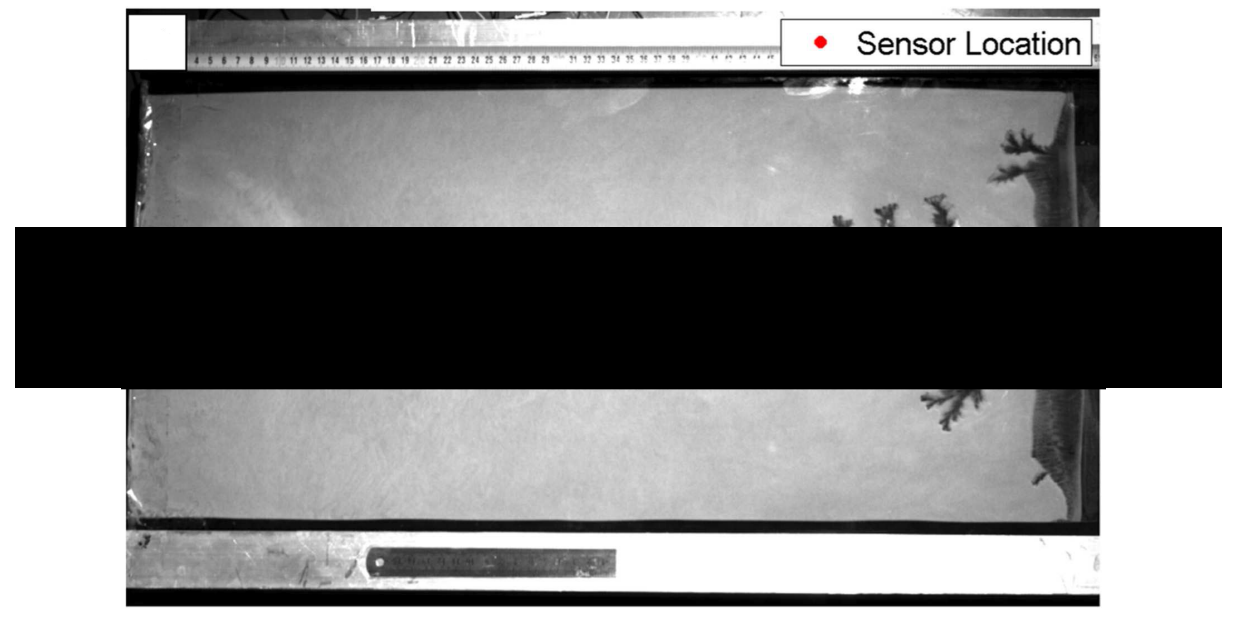

Figure 3.5: Image of two-phase aero-fracture experiment performed by Turkaya et al. (2015) [77]. The red dot represents the location of an acoustic sensor.

drop across a bead diameter), and the resisting forces $\Delta f_{r}$ (estimated using the friction force caused by the confining pressure)

$$
N_{f}=\frac{\Delta f_{p}}{\Delta f_{r}}=\frac{\gamma L^{2}}{\mu w d}(1+C a) .
$$

Where $\mathrm{Ca}$ is the capillary number, $C a=\eta v / \gamma$, with the characteristic flow velocity estimated as $v \sim Q /(b d)$. For $N_{f} \ll 1$ the system is practically rigid and invasion occurs without disturbing the packing, while at $N_{f}>1$ the packing is deformable and fracturing occurs. They also found that, for these experiments, this transition did not depend on flow rate $Q$ as $C a \ll 1$ and so the capillary pressure was the primary cause for fracture initiation.

Fracturing dynamics in dense granular media have also been specifically studied previously in a Hele-Shaw cell with two-phase regimes, i.e where the invading fluid and the interstitial fluid are the same. This is usually performed with air and polystyrene beads, see Figure 3.5 [77, 78]. The observed patterns are long and slender and were described as fractures since force chains (frictional contacts between the beads and the confining plates) needed to be broken in order for branches to grow [78]. Two-phase fracturing experiments and their modelling are motivated by industrial hydraulic fracturing, where pressurised water is injected into rock formations [79]: acoustic measurements taken of pneumatic polystyrene bead fractures have recorded frequency signals that are comparable to initial seismic activity caused by hydraulic fracturing as well as its after shocks [77]. 
This thesis aims to fill a niche in literature with an examination in Chapter 4 of specifically how growing multiphase fracture branches in a Hele-Shaw cell deform the settled granular medium around them and how this deformation influences future pattern growth. In Chapter 5 a model will be described that explains the patterning behaviour and how invasion pattern characteristics can be manipulated.

\subsection{Fracturing in Suspended Granular Media}

Two examples of work that has real world applications for which it is critical to have a further understanding of multiphase flows in suspended granular media will be discussed here. The first is the natural phenomenon of magma flows and the second is an industrial application with soft body armour.

\subsubsection{Magma Flows}

Magma often contains crystals, making it a granular suspension. Migration of gas (volatiles) through a mush of crystals and magma influence eruption behaviour and the escape of large amounts of gases to the atmosphere [80, 81]. Gas-driven fracturing and deformation of the crystal matrix creates conductive pathways for migration of volatiles through the mush, which can explain the episodic eruptive bursts characteristic for some volcanic systems, and why crystal rich magmas often are found to have depleted volatile fractions [9, 81, 82]. Understanding the rheological properties and flow characteristics of magma when subject to gas invasion and other stresses is important for studying tectonic motion, volcanic eruptions, and seismic influence on volcanic activity [8, 83, 86].

While at rest, dense crystal-bearing magma have a high viscosity, resisting flow due to friction between crystals. When subject to motion the material shear thins and flows more readily. However, if subject to excess motion the magma dilates, and its crystals can interlock causing shear thickening [8, 87, 88]. In natural circumstances, seismic activity can cause the requisite motion, or shearing, for local magma viscosity to be changed. It has been proposed that seismic induced shear thinning of magma can allow for flow that leads to volcanic eruptions; explaining why an earthquake can be followed by a flurry of volcanic activity [85]. Whereas magma shear thickening is important for understanding blockages that can lead to increases in pressure and internal fracturing [87]. 
It should be noted that the viscosity of magma is also temperature dependant, higher with lower temperatures and lower with higher temperatures, making it difficult to isolate variables in a stress based rheological context [85, 86]. Of course, the study of the rheology of magma suspensions has also been significantly hindered by its dangerous nature [8, 85]. Volcanologists have studied the textures and surface patterns of igneous rocks for evidence of magma flow dynamics and rheology, inferring crystal alignments and dilatancy processes that suggest shear thickening [85, 88]. Analogous system experiments have been performed using silicone oils and beads to represent the crystal-bearing magma; these have shown a regime transition from thinning to thickening as a function of increasing shear in suspensions with particle concentrations from $\phi=0.2-0.6$ [8]. Recently, rheological equipment has become advanced enough to perform rheological readings on real magma samples with crystal concentrations from $\phi=0.55-0.65$ and have confirmed these previous findings [87]. However, experiments involving magma flow still require model replacement fluids [9, 82].

\subsubsection{Soft Body Armour}

Modern body armour is split into two designs: hard and soft. Hard body armour is made with ceramic or steel plates and designed to protect the torso from high-speed bullets for military personnel in high-risk environments [89]. More flexible and lighter than hard body armour, soft body armour is made with multi-layered woven of laminated fabrics and designed to protect police officers and security personnel against lower ballistic threats such as handguns and shotguns [89]. Despite the fibers having high strength, low density, and high energy absorption, the numerous layers of the fabrics required to stop a bullet result in a still relatively bulky and stiff armour that is restricted to protecting the torso [90]. As such, there is great interest in producing lighter armour, with equivalent protection, that is flexible enough to be worn on appendages [91]. 


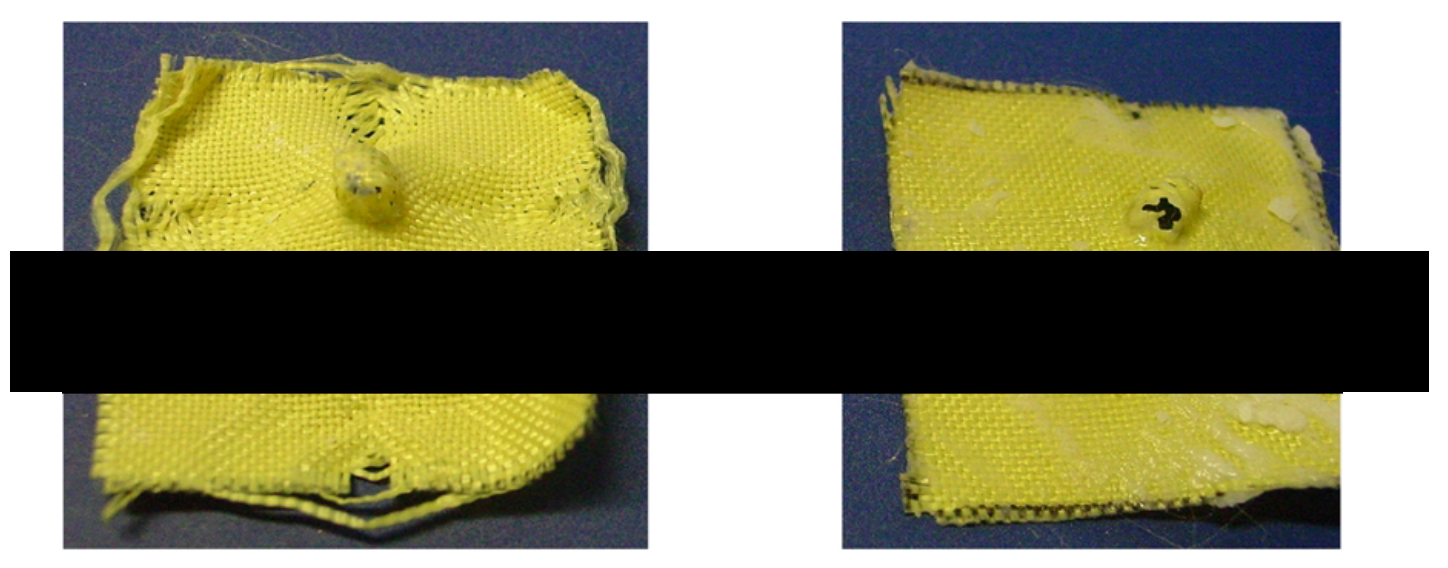

(a)

(b)

Figure 3.6: Image of Kevlar ballistic performance tests by Lee et al. (2003) [90]. a) Kevlar material exhibits significant stretching from ballistic impact. b) Kevlar material impregnated with shear thickening fluid exhibiting no stretching and less penetration depth from ballistic impact.

It has been proposed that high performance fabrics could be impregnated with a dense granular suspension to exploit DST behaviour while reducing the number of layers needed. During the normal motions made by the wearer, the fluid will experience low shear rates and make little resistance to the fabrics flexibility; however, upon impact with a bullet a DST response will be invoked, which means that that the viscosity of the fluid suddenly rises orders of magnitude when a critical shear rate is reached, thus increasing its effectiveness against penetration. Initial studies with impact velocities below $300 \mathrm{~m} . \mathrm{s}^{-1}$ were successful, showing soft body armour using a shear thickening fluid could sufficiently protect a target with much thinner and more flexible designs than traditional armours, see Figure 3.6 [90, 92-94]. However, both handgun and shotgun projectiles have impact velocities exceeding $300 \mathrm{~m} . \mathrm{s}^{-1}$. For this range the reported benefit of shear thickening fluids is mixed [95]. Many experiments indicate a $20 \%$ enhancement to fabric performance, which is significantly lower than the maximum $200 \%$ enhancement observed for sub-200 m.s ${ }^{-1}$ tests [96-99]. Other experiments report equivalent or reduced results for impregnated fabrics compared with unaltered ones [95, 100, 103]. It has also been reported that the more layers included the less useful the shear thickening fluid becomes, as the jump in viscosity hinders the ability of the multiple fabric layers to move with the impact [95]. It should be noted though, that these are preliminary experiments that have not used DST fluids or applications of DST fluids that have been optimised for this purpose. With further understanding of how shear thickening fluids work and behave enhanced results can be obtained. 


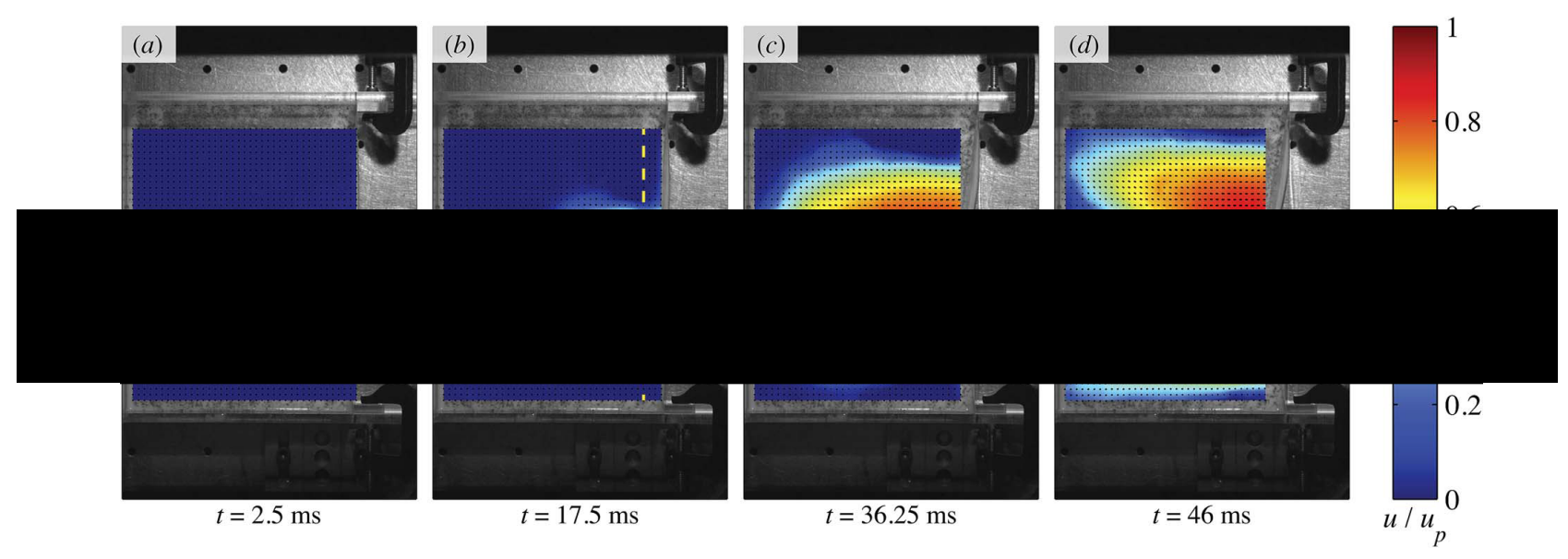

Figure 3.7: PIV analysis of cornstarch suspension impact experiment by Peters et al. (2014) [122]. Color bar represents suspension velocity normalised with the impact velocity, with images showing growth of jamming front.

\subsubsection{Rheology of Suspensions}

A key difference between suspended and settled granular systems is that the grains in a suspension readily move with the suspending fluid. In other words, the granular matter is coupled with the flow and viscosity of the fluid. As such, a conventional way to study the properties of a suspension is with a rheometer. Rheometric studies have revealed that granular and colloidal suspensions exhibit a wide variety of complex non-Newtonian flow behaviours. Micro-scale grain-grain and grain-fluid interactions can, under different circumstances, manifest as a yield stress, thixotropy, jamming, shear thinning, or shear thickening; the latter of which is the focus for this thesis [104-109].

Cornstarch mixed with water provides a readily available classic example of a granular suspension that exhibits shear thickening and DST behaviour. In fact it can become so resistant to motion that a person can run across the surface of the "fluid" [110]. Note that this behaviour is reversible: the suspension begins to relax and flow again after the stress is removed. Cornstarch particles are inert and hard, with a Young's modulus of approximately $10 \mathrm{GPa}$ [111]. Its most pronounced characteristic is that it is highly hygroscopic (it attracts and absorbs water molecules), indicating marginal particle-water surface tension [112]. Cornstarch particles are around $10-20 \mu \mathrm{m}$ in size, which is convenient as smaller particles are dominated by Brownian motion and larger ones readily settle under gravity [112].

As a consequence, cornstarch suspensions have been routinely used as a model sample for experiments designed to study the DST phenomenon. Experiments have advanced our understanding of shear thickening through detailed rheological studies [110, 113[16], careful characterization of impact generated shear jamming fronts (see Figure 3.7) [117-124], and benchmarking of DST models [125-127]. 


\subsubsection{Proposed Mechanisms for Discontinuous Shear Thickening}

A mechanism for DST needs to explain a number of recorded properties. The DST phenomenon only occurs in volume fractions close to the random close packing limit of the system, below this weak continuous shear-thickening is observed. DST is observed in hard-particle suspensions, but not with soft-particles, emulsions, or attracting colloids. The DST regime tends to begin at a critical minimum stress that is approximately independent of volume fraction. The DST behaviour results in the viscosity increasing by orders of magnitude. The DST regime ends at a maximum stress that is also approximately independent of volume fraction [113, 128,-130]. Historically, a number of mechanisms for DST have been proposed; it is of interest to outline these mechanisms and what DST behaviour they did not fully explain as this then influenced what later experimental work focused on.

One proposed mechanism was order-disorder transition. Order-disorder transition is a process by which the viscosity of a fluid increases as a result of the structure of particles in the flow altering from ordered layers into a disordered structure with shear [131]. Though microstructural reorganisation has been observed to coincide with shear thickening, modern rheological studies have reported that an order-disorder transition is not required for DST [132-134].

Another proposed mechanism was hydroclustering. Hydroclustering is a process by which the lubrication drag forces holding particles together increases as particles cluster under shear [135, 136]. Hydroclustering can predict the minimum stress at which shear thickening occurs and has been observed during the initiation of shear thickening [137]. However, the stresses measured during DST are significantly too high to be the result of lubrication forces [112].

The stresses reached during DST are more readily explained by force chains that reach through the system. Much like granular matter, these force chains can support surprisingly large stresses that are of a magnitude similar to those observed during DST [138]. Following this, a third proposed mechanism is confined dilatancy. Dilatancy is a process in which the particle packing expands under shear. Dilatancy is a well known phenomenon in dense granular flows, as a suspension is sheared the grains must move over and around each other to allow flow, and thus the volume they occupy increases [139, 140]. Dilatancy has long been reported to occur with DST and was even used as a synonym for it until Metzner and Whitlock showed that dilatancy could be found to occur without a DST response [139, 141]. It has been proposed that dilatancy in a confined suspension under shear causes the particles to push against the boundaries, forming force chains that jam the system resulting in discontinuous shear-thickening [114]. 
Unlike order-disorder transition and hydroclustering, the dilatancy model has been developed enough to predict the upper stress limit on shear thickening as well as the lower limit [105, 114, 142]. The lower limit is set by the shear stress required to rearrange two close particles for dilation. The upper limit is set by the weakest boundary limits, typically the surface tension of the interstitial fluid which can be overcome by dilating particles at high enough shear. It also explains why soft particles (such as emulsions) do not exhibit DST behaviour: they can shear without dilating since the particles themselves will deform before a significant stress is exerted on them by the confining system. However, this model has not yet qualitatively predicted rheological flow curves.

\subsubsection{Friction Transition}

A more recently proposed mechanism is a frictional transition. This is a process by which a repulsive force between particles prevents particle contact at low stresses but interparticle pressure overcomes the repulsion at higher stresses, allowing frictional contacts to be made and thus greatly increasing the viscosity of the system [125, 127, 143, 146]. The great increase in viscosity is sudden because the repulsive force prevents a more gradual breakdown of lubrication films [123, 147]. Like dilatancy, this model also explains why neither emulsions nor attractive colloids shear thicken. Emulsions are always lubricated and do not experience a frictional state, where as attractive colloids are always frictional and do not experience a lubricated state. Some simulations have recently affirmed that friction is required for DST to occur [127, 144, 148, 149]. This model has also qualitatively predicted rheological flow curves and will be outlined in Chapter 6 [125, 146].

As a result of this new proposal, recent innovative experiments have examined the frictional nature of cornstarch particles, and the role of inter-particle pressure in dense granular suspensions. However, theoretical and experimental analysis of macroscopic DST behaviour and flows in confined geometries other than the classic rheometry tools remain scarce [150].

Comtet et al. (2017) [151] measured the frictional profile of cornstarch particles with new experimental methods to test whether a short range transition to frictional behaviour is present. Using quartz-tuning force atomic microscopy they reported on a transition from a low-friction regime to a high-friction regime at a critical normal stress in the nanoscale that coincided with the critical stress for which shear thickening occurred in macroscopic samples. They state there is "a clear view of discontinuous shear-thickening transition as the breakdown of lubricated contact between particles, at a critical normal force." 
Conventional rheological methods do not give insight to the frictional nature of a suspension. However rotating drums have previously been used to examine friction in dry granular flows by measuring avalanche angles under gravity, which represents a dynamic angle of repose [152-155]. Clavaud et al. (2017) [156] utilised a rotating drum with varying concentrations of cornstarch suspensions to measure the avalanche angle as well as any compaction or dilatancy effects under a low confining stress (gravity).

In contrast to Newtonian suspensions of frictional particles [157, 158], they found that the cornstarch suspensions had a very shallow avalanche angle and did not compact or dilate. From this behaviour they determined that the cornstarch suspensions existed in a frictionless state while under a low confining stress. In addition, they also used a suspension of negatively charged silica beads for which they could control the range of the repulsive force. At a low confining stress, once the range was smaller than the particle roughness, the suspension transitioned from frictionless to frictional. The suspensions for which the frictionless state was not present also had a suppressed shear thickening response to larger stresses. As a result, they confirmed that this frictional transition caused by repulsive forces preventing inter-particle contact is necessary for DST behaviour.

To explore this frictional transition accurately in a rheological context, the pressure experienced by the particles in the suspension needs to be examined, as opposed to the stress experienced by the suspension as a whole which rheology conventionally deals with. In a confined geometry the inter-particle pressure is exerted upon the walls of the system, however separating this from the fluid pressure that is also experienced by the walls is the difficulty.

Boyer et al. (2011) [159] designed a custom-built rheometer for this purpose. The sample is sheared between two parallel plates, except the top plate is a grid through which the interstitial fluid could flow freely but the particles cannot. In addition, the horizontally shearing grid-plate is free to move in the vertical axis but has a controlled constant force applied to it. Therefore, its vertical height changes according to the particle pressure it experiences, as opposed to the suspension or fluid pressure. In this setup the volume fraction of the confined suspension is free to change and is no longer a controlled factor, instead the confining stress exerted by the top grid-plate on the particles is an experimental parameter and the particle pressure is measured.

This new design enables accurate particle pressure readings while approaching the jamming volume fraction limit of a suspension, since controlling the confining particle stress and gradually lowering the shear rate to reduce the volume fraction of the system is far more convenient than performing multiple experiments with suspensions of differently prepared volume fractions. Of course, if you need to perform rheological measurements for suspensions of specific volume fractions (as is the case in for Chapters 6 and 7 in this thesis) then conventional rheological techniques are more suitable. 


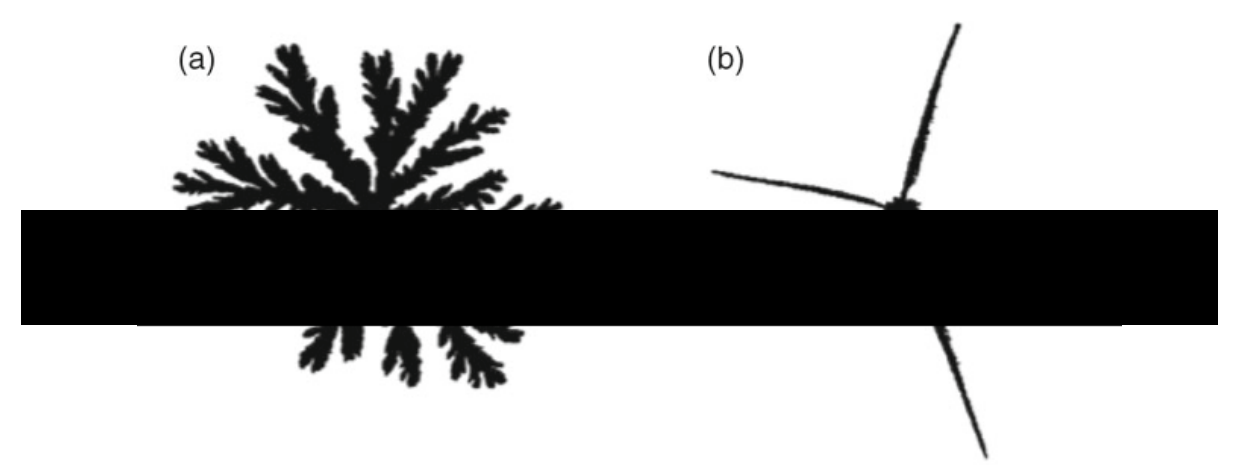

(c)

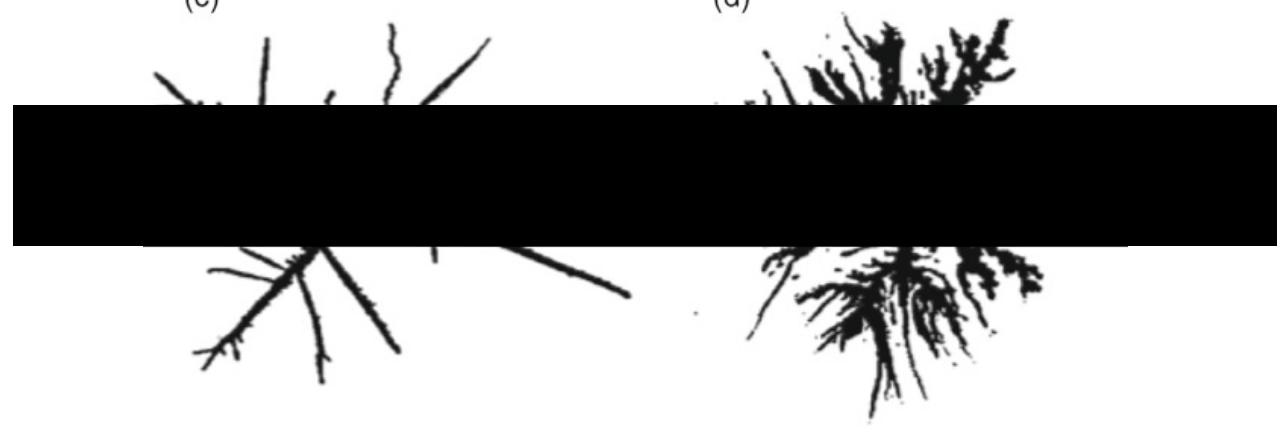

Figure 3.8: Hele-Shaw invasion patterns illustrating transition from fingering to fracturing in visco-elastic polymer solutions by Zhao et al. (1993) [161]. Solution of $2.5 \%$ of hexadecyl-end-capped polymer subject to injection of water at rates of a) $\mathrm{Q}=$ $1.0 \mathrm{ml} / \mathrm{min}$, b) $\mathrm{Q}=1.0 \mathrm{ml} / \mathrm{min}, \mathrm{c}) \mathrm{Q}=5.0 \mathrm{ml} / \mathrm{min}$, and d) $\mathrm{Q}=20 \mathrm{ml} / \mathrm{min}$.

Unfortunately, this setup has yet to have been used with a suspension of cornstarch particles. However, it illustrates the difference between the shear stress of the suspension and inter-particle pressure. Boyer et al. (2011) [159] also determined that the shear stress is proportional to the inter-particle pressure and an effective macroscopic coefficient of friction (not the inter-particle friction). This new method of rheometry highlights the importance of particle contact and pressure when discussing dense granular suspensions and opens the door to pressure and stress based descriptions of their rheological properties instead of the traditional volume fraction based descriptions [160].

\subsubsection{Complex Suspensions and Hele-Shaw cell}

Hele-Shaw cell invasion experiments can be used to observe the transitions of rheological behaviour in a defending complex fluid by controlling the invasion rate or pressure of the invading fluid. Though the setup is not capable of the precise rheological measurements of a rheometer, the resultant visualised invasion patterns can offer great insights into the mechanical response and behaviour of the fluid to different shear rates and stresses. 
There is a rich literature of Hele-Shaw invasion experiments used with viscoelastic fluids [161-169]. Like DST suspensions, viscoelastic materials can transition from a liquid-like state to a solid-like state, except here the transition is a result of the timescale of the interaction instead of stress. The Deborah number $D e$, a dimensionless constant associated with viscoelastic fluids, describes this behaviour. The Deborah number is a ratio between the characteristic relaxation time of the viscoelastic material $\tau_{r}$ and the characteristic flow time of an event $\tau_{f}$. When $D e \gg 1$ the relaxation time is larger, elastic effects dominate and the behaviour is solid-like; when $D e \ll 1$ the flow time of an event is larger, viscous effects dominate and the behaviour is liquid-like.

This transition to solid-like behaviour includes a flow-to-fracture transition. Fracturing behavior in viscoelastic fluids was first reported in the 1960s [163]. The most commonly studied viscoelastic material for flow-to-fracture transitions are transient networks of cross-linked polymers in suspension as they do not have a yield stress [162]. In addition to Hele-Shaw cell experiments, rheometry [164, 165, 170, 171] and droplet fall experiments [170, 172] have been used to study flow-to-fracture transitions in viscoelastic polymer networks. Rheometry results show that viscoelastic polymer networks, as a function of increasing shear rate, generally have an initial Newtonian viscosity, some then exhibit continuous shear thickening, and all then experience a drastic shear thinning regime at a critical shear rate [164, 165, 170, 171].

This critical shear rate also marks where the transition from viscous fingering to fracturing is observed in Hele-Shaw experiments for viscoelastic polymer networks [161, [164-168], see Figure 3.8. The fractures are reversible and the initial state is recovered after a relaxation period. It was established in the 90 s that how the suspended polymer network responds to an imposed shear is governed by the Deborah number, which can be raised by increasing the injection rate of the invading fluid assuming the geometry of the system is the same [161, 166, 173]. As a result of this rate dependency, a viscoelastic fluid with an applied shear stress will eventually fracture [165]. Lowering the stress below the critical stress at which it immediately fractures merely increases how long it takes for fracturing to be observed. This is similar to pressure driven granular systems, such as the pockmarks mentioned earlier in Section 3.1.2. This is a key difference between DST suspensions and viscoelastic fluids as DST systems will not exhibit solid-like behaviour until a critical stress is reached. 
Previously, Kagei et al. (2005) [174] used a Hele-Shaw cell to study viscous fingering in shear thickening (but not DST) silica suspensions. They found that the behaviour before thickening was similar to descriptions in the viscoelastic polymer literature but did not observe the fracturing that is reported there at higher shears. The flow-to-fracture transition for DST suspensions has not yet been explored with Hele-Shaw invasion experiments. This thesis aims to fill this niche in literature with a study on Hele-Shaw invasion patterns in DST suspensions, while measuring how the imposed pressure and suspension concentration influences the invasion pattern characteristics. Wyart-Cates' model, as outlined in Chapter 6, will also be employed to see how well the theory explains nonrheometric experimental data. 


\section{Chapter 4}

\section{Fracture Formation at the Granular Scale}

\subsection{Introduction}

Here new insights into the motion of the packing at the granular scale during individual fracturing events will be introduced. First an example of multiphase fracture patterns formed in a larger Hele-Shaw cell and an illustration of the stick-slip nature of the growth process on a time-scale of minutes will be presented. Then a high-speed camera and image analysis is used to examine the motion of the fracture and its surrounding grains in a smaller Hele-Shaw cell. The high-speed imaging revealed additional frictional intermittent behaviour at significantly shorter time-scales, and allowed the use of Particle Image Velocimetry (PIV) to calculate displacement, velocity, and compaction fields for the deformation of the granular packing surrounding the growing fracture. Data from multiple fracturing events is used to generate an average velocity profile of fracture growth for the system, as well as a subsequent average compaction profile. These profiles help explain many of the interesting features of multiphase fracture patterns, including how local granular compaction induces stick-slip behaviour and creates a compacted zone surround the fractures that other fractures cannot penetrate, determining the characteristic spacing between them.

The primary highspeed experiment in this chapter was designed, conducted, and analysed by myself with the supervision of Bjørnar Sandnes. The larger experiment described in this chapter for context was designed by James Campbell and Bjørnar Sandnes but the results and analysis shown from it were performed by myself witht he supervision of Bjørnar Sandnes. 


\subsection{Materials and Methods}

\subsubsection{Fracture Pattern Generation}

The branching fracture patterns presented here were created in experiments using a HeleShaw cell, see Figure 4.1. Impermeable adhesive plastic spacers were used to hold two $350 \times 350 \times 18 \mathrm{~mm}^{3}$ glass plates apart, creating a $200 \mathrm{~mm}$ wide, $300 \mathrm{~mm}$ long channel with a $0.5 \mathrm{~mm}$ gap height. One end of the cell was open to the surrounding atmosphere, and a hole for an inlet was drilled $300 \mathrm{~mm}$ from the open end.

The cell was filled with water-saturated, loosely packed spherical soda-lime glass beads, sieved to a size range of $0.20-0.25 \mathrm{~mm}$. The cell was filled by turning it vertical, flooding it with deionised water and then pouring in grains quickly and evenly across the channel width, ensuring the cell was constantly filled with gradually settling grains. The grains were filled up to $10 \mathrm{~mm}$ below the open end of the channel, with the remaining space filled with water, preventing evaporation from affecting the granular packing during the experiment.

a)

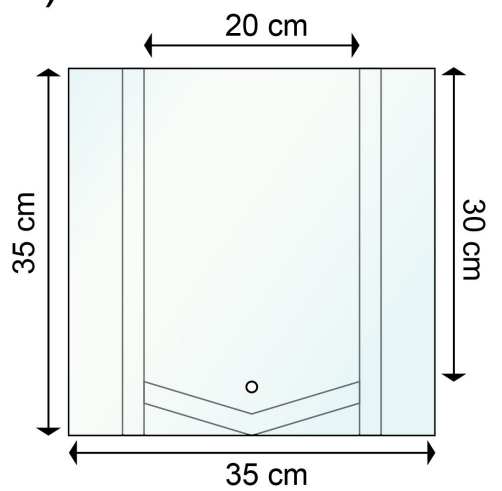

b)

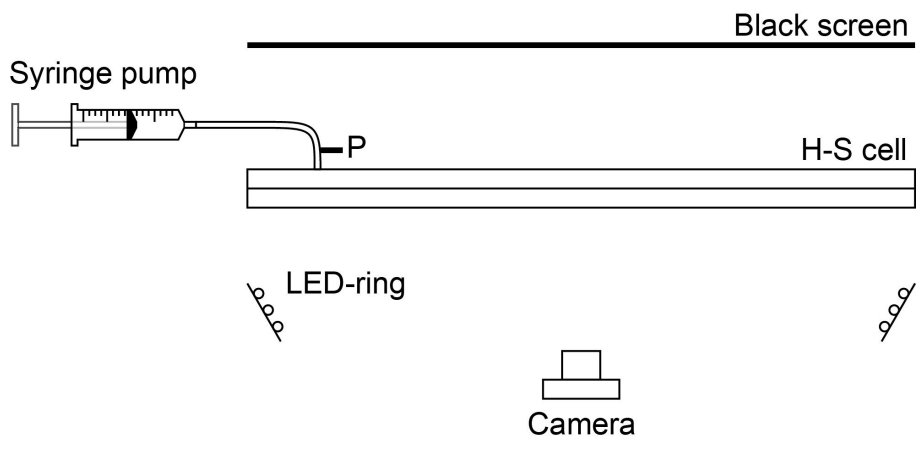

Figure 4.1: a) Schematic of larger Hele-Shaw cell. b) Air is injected into Hele-Shaw cell via syringe pump to form fractures in a water-saturated, loose granular packing held in the cell with a gap size of $0.5 \mathrm{~mm}$. Air pressure is recorded just before the inlet. $Q_{\text {pump }}=0.01 \mathrm{ml} . \mathrm{min}^{-1}$, grains used for packing were sieved to $0.20-0.25 \mathrm{~mm}$, photo taken from below every $20 \mathrm{~s}$. 
Once the bed had settled the cell was turned horizontal. A syringe pump (Harvard Scientific, PHD Ultra) was used to inject air through the cell inlet into the water-saturated granular packing confined in the cell gap. The pressure of the air was recorded at $5 \mathrm{~Hz}$ during injection with a difference pressure sensor (Sensortechnics DC13) connected to a National Instrument-9171 analogue input DAQ module. The air was injected at a rate of $Q_{\text {pump }}=0.01 \mathrm{ml} \cdot \mathrm{min}^{-1}$ from an air reservoir of $V_{\text {gas }}=8 \mathrm{ml}$ contained within a syringe. The development of the fracture pattern was photographed with a camera (Nikon J2) every $20 \mathrm{~s}$ with an image resolution of $3872 \times 2592 \mathrm{pixel}^{2}$. Typically the experiment would run for up to 30 minutes as the fracture pattern explored the cell until it reached the end of the packing near the cell opening. Following Equation 3.1, this system has an estimated Capillary number of $5 \times 10^{-6}$ which is a suitable creeping flow.

Prior to use, the beads were washed in a 3:1 mix of sulphuric acid and hydrogen peroxide and then rinsed in deionized water to ensure clean hydrophilic surfaces with an estimated air-water-glass contact angle of $\theta=12 \pm 5^{\circ}$ [175]. The filling procedure and gentle sedimentation ensured that the beads were loosely packed and that the packing was deformable. The bead beds had an estimated porosity of $n=0.47$, which is concordant with the expected values for a random loose packing of spheres [176]. The large scale phenomenon of the bed porosity was calculated by measuring the volume of the cell that was filled by a significantly large known mass of the granular material.

\subsubsection{Fracture Pattern Image Analysis}

The images taken during the experiment were used to investigate the overall pattern formed by the gas-driven fracture growth. The images were processed by a custom algorithm in MATLAB. The algorithm uses an image taken of the bed before fracturing began and the final image from when the experiment ended as input data along with calibration numbers for distances. The final image was subtracted from the initial image to eliminate pixels that had not changed through the experiment i.e. regions of material that had not moved. This left an image showing material that had moved which was then thresholded to make the data binary and eliminate small differences between the images i.e. regions of minor motion. The threshold value was kept consistent between experiments. 


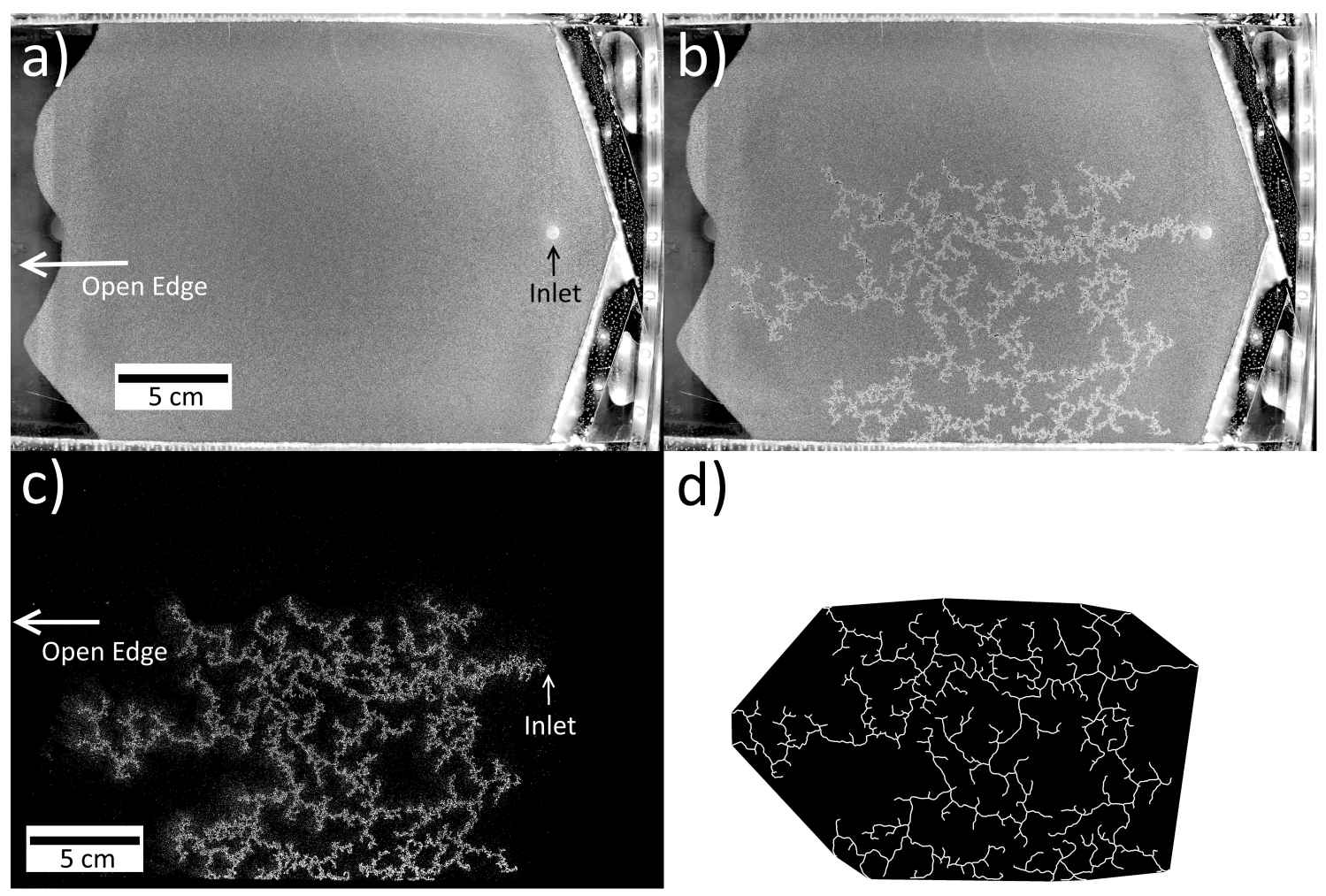

Figure 4.2: Fracture invasion pattern in larger cell. a) Raw image of granular packing before gas invasion. b) Raw image of granular packing after gas has invaded forming a fracture pattern. c) The result of subtracting (a) from (b). d) Processed and skeletonised fracture path with its surrounding convex hull in black.

This process clearly reveals the fracture cavities but also captures a halo of motion around the fractures which need to be eliminated to analyse the fracture network, see Figure 4.2 (c). To do this a number of techniques were used in the algorithm. As the fracture network is continuous it represents a large series of connected data whereas as the halo of motion captured around them are made of numerous but significantly smaller clumps of data. So despeckling was applied, which removes clumps of connected data that are below a certain size. Next a closing operation is performed, which fills in empty regions that are entirely surrounded by data. This was done to fill in the fractures and to reveal any closed loops within the fracture network. Closed loops were never observed during experimentation and instead represent erroneous data from the halo of motion not successfully eliminated by the thresholding and despeckling. At this stage the data was checked for those closed loops and any found where manually eliminated by removing the excess data that was connecting two fractures by comparing to the original image after which the closing operation was reperformed. 


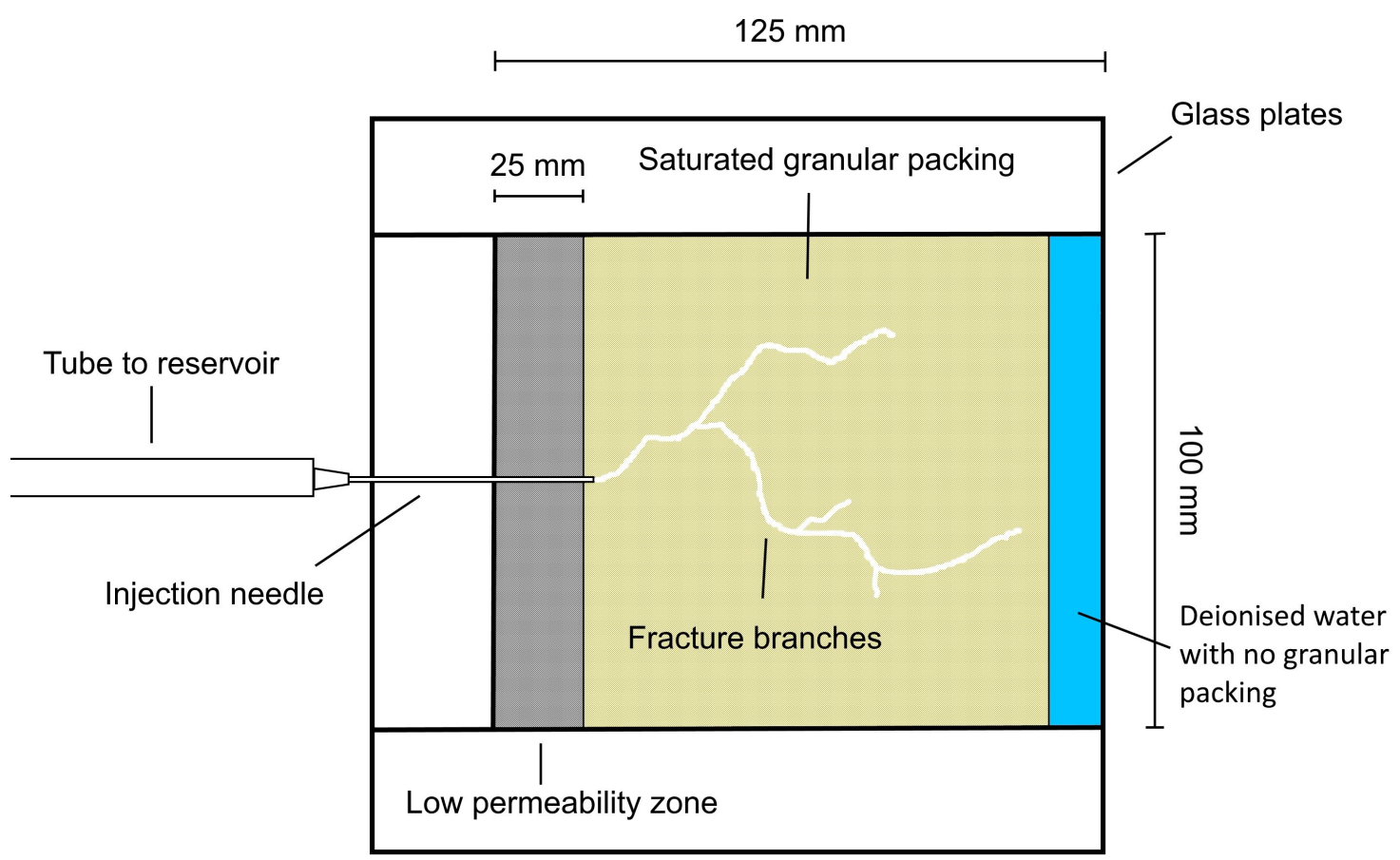

Figure 4.3: Small Hele-Shaw cell. Air is injected through a syringe needle to form fractures in a water-saturated, loose granular packing held in the cell with a gap size of $0.9 \mathrm{~mm} . Q_{\text {pump }}=0.01 \mathrm{ml} \cdot \mathrm{min}^{-1}$, grains used for packing were sieved to $0.20-0.25 \mathrm{~mm}$, grains used in low permeability zone were sieved to $0.10-0.15 \mathrm{~mm}$, high-speed camera filmed from below at $1000 \mathrm{fps}$.

This process can "erode" the boundary of the fractures, changing the width of them. However, this is not an issue as it is the length of the fractures that is needed, not the width. To obtain this the isolated fracture network is skeletonised. Skeletonisation reduced the structure to a series of singular connected pixels, see Figure 4.2 (d) i.e. a structure with a width of one pixel. Counting these pixels gives the length of the fracture network. The two-dimensional spatial density of the fracture patterns was calculated by dividing this skeletonised fracture length by the area of the convex hull of the pattern (the area occupied by a perimeter defined by straight lines between its most extreme points), see Figure $4.2(\mathrm{~d})$. 


\subsubsection{High-speed Setup}

A smaller Hele-Shaw cell was constructed for close-up high-speed imaging to reduce the chance of fractures growing off camera, see Figure 4.3 . Two 150x150x10 mm $\mathrm{m}^{3}$ glass plates were held apart by impermeable plastic adhesive spacers of $0.9 \mathrm{~mm}$ thickness, defining the cell gap. Like the larger cell, the cell was left open at one end and the resultant channel formed was $100 \mathrm{~mm}$ wide and $125 \mathrm{~mm}$ long. A syringe needle was inserted through the plastic spacer, reaching $25 \mathrm{~mm}$ into the cell opposite the open end. The needle tip formed the air inlet, driven by a syringe pump (Harvard Scientific, PHD Ultra) at constant injection rate of $Q_{\text {pump }}=0.01 \mathrm{ml} . \mathrm{min}^{-1}$ with a reservoir volume $V_{\text {gas }}=8 \mathrm{ml}$.

Glass beads $(0.20-0.25 \mathrm{~mm})$ were used to form a granular packing as described for the larger cell, leaving a $10 \mathrm{~mm}$ water filled gap at the open edge. However, an initial layer of smaller 0.10-0.15 mm beads was first deposited in the cell to form a low-permeability zone at the bottom of the channel next to the syringe needle. This was done to prevent the fractures from growing back along the needle and along the bottom edge of the cell outside of the filmed area, since edge effects between the larger grains and the syringe needle create a high permeability zone compared with the rest of the bed. Similarly to the larger Hele-Shaw cell, this system has an estimated Capillary number of $5 \times 10^{-6}$.

The high-speed camera (Photron Fastcam 1024-PCI) had a resolution of 1024x1024 pixel $^{2}$, and was set to film a 50x50 $\mathrm{mm}^{2}$ area of the Hele-Shaw cell channel giving an approximate resolution of $6 x 6$ pixel $^{2}$ per bead. The camera filmed with a frame rate of $1000 \mathrm{fps}$. The $50 \times 50 \mathrm{~mm}^{2}$ frame was large enough to capture all motion of the granular bed in the vicinity of an individual fracture event. The longest fracture event observed lasted for $1 \mathrm{~s}$ and the shortest for $0.13 \mathrm{~s}$.

\subsubsection{High-speed Image Analysis}

The videos taken with the high-speed camera were used to investigate the deformation and motion of the granular packing around individual fractures. Displacement and velocity vector fields of the granular motion were found using Particle Image Velocimetry (PIV) performed using Ncorr, a MATLAB based digital image correlation (DIC) software [177]. PIV is a process in which sub-windows of an image are cross-correlated with sub-windows of another image taken at a later time to obtain displacement vectors $\vec{S}(x, y)=X(x, y) \vec{i}+Y(x, y) \vec{j}$ located at $(x, y)$ positions in the initial image. These displacement vectors are then calibrated with the distance across each sub-window $(0.15 \mathrm{~mm})$ and the time between images $(1 \mathrm{~ms})$ to obtain the displacement and velocity vector fields. 


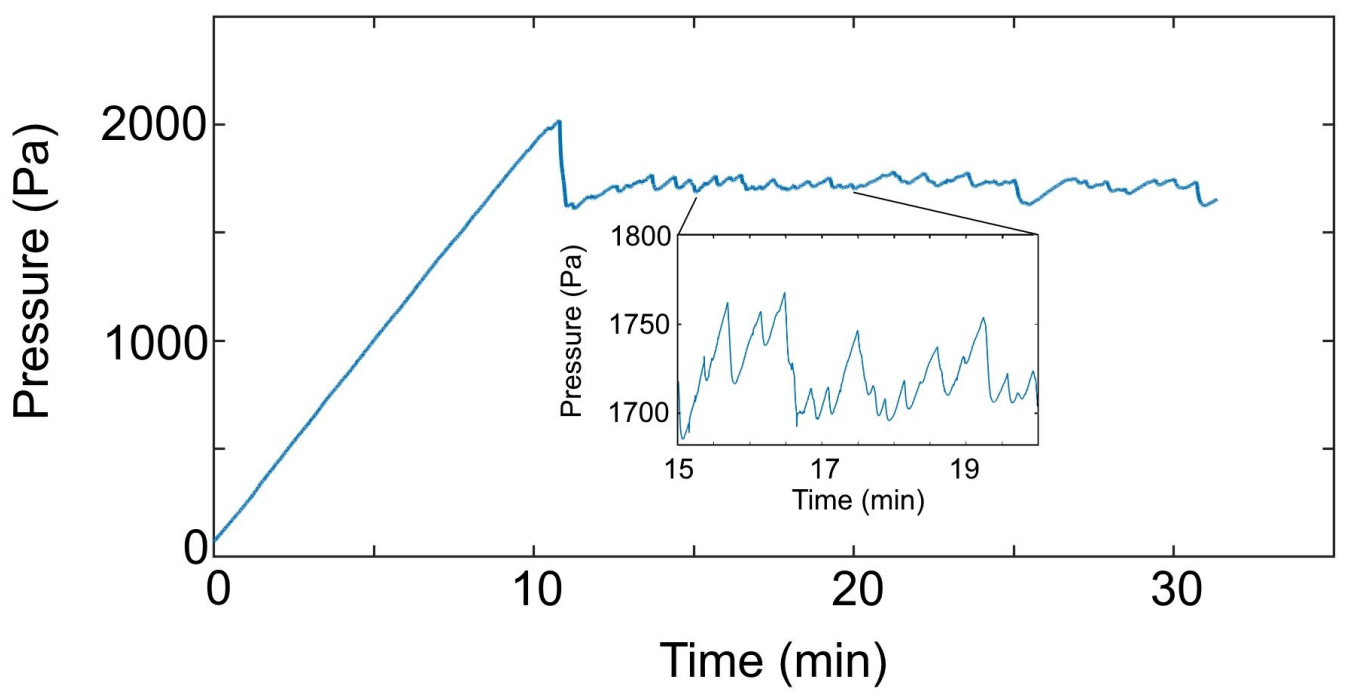

Figure 4.4: Air pressure curve during gas-driven fracturing of saturated granular media in the large cell. The initial linear increase is a result of gradual compression of air at steady pump rate prior to fracturing. The first sudden drop corresponds to the first invasion through the inlet. Thereafter, the gas pressure displays a saw-tooth shape where the peaks correspond to the pressure required to initiate a new fracture, the local pressure minima correspond to the end of a fracture growth event, and the steady increase correspond to the "stick" period between fracture events, where the gas compresses at the rate dictated by the pump. Inset: Subsection of curve, illustrating the saw-tooth shape of the pressure during stick-slip fracture growth. (Injection rate $q=0.01 \mathrm{ml} . \mathrm{min}^{-1}, 0.20-0.25 \mathrm{~mm}$ beads.)

The average velocity profile of the filmed fracturing events presented here was found using a custom MATLAB algorithm that selected frames from a high-speed video in which the PIV data contained a minimum threshold of movement, an average mean motion of $0.5 \mathrm{~mm} . \mathrm{s}^{-1}$ in the $50 \times 50 \mathrm{~mm}^{2}$ frame was found to appropriately select frames with active fracture growth. Those velocity fields depicting growth were then re-centred to the location of their fracture tip, which was reliably found by correlating the directions of the largest 50 vectors in the frame to find their overlapping point of origin, defined here as the fracture tip. This method relies on the observation that the deformation field spreads out approximately radially from the tip of the fracture. These translated fields were then rotated to the same vertical orientation by determining their overall angles of motion, which was found by the mean angle of those same 50 largest vectors, defined here as the direction of fracture growth. The PIV data found at this fracture tip was used to find the fracture tip speed for that frame. 783 processed velocity fields from multiple fracturing events were averaged to find the contour plot presented in Figure 4.8 later in Section 3. 
The compaction of the granular medium porosity $\Xi$, relative to the original packing porosity, around fracture growth was calculated with a custom algorithm in MATLAB using a larger sub-window $(0.58 \mathrm{~mm})$, to enable the cross-correlation of multiple grains at once. The algorithm looks at the vectors perpendicular to the boundaries between the sub-windows left, right, above, and below the sub-window in question. It checks whether those vectors are facing into the sub-window (compaction) or if they are facing away from it (decompaction). It then totals those four components (left, right, above, and below) to find if the sub-window is overall compacted or decompacted and by how much.

$$
\Xi=\left(\Delta X_{i}+\Delta Y_{j}\right) \cdot \frac{100}{L}
$$

$\Delta X_{i}$ is the difference between the $\vec{i}$ components of the displacement vectors found on the left and right sides of the sub-window, $\Delta Y_{j}$ is the difference between the $\vec{j}$ components of the displacement vectors found above and below the sub-window, and $L$ is the length across the sub-window.

\subsection{Results and discussion}

\subsubsection{Fracture Pattern}

This section outlines the features of the patterns formed by pneumatic fracture growth in a deformable saturated granular media in the large cell described in Section 2.1. Figure 4.2 (b) shows an example image of an invasion pattern in this setup, while (d) shows the skeletonised path of the pattern that was used for analysis. As the air invades the saturated packing it creates fracture pathways that branch by tip-splitting. The resultant fracture pattern forms a maze: a branching, loopless, binary tree-like structure. The reason for the self-avoiding nature of the fracture network cannot be discerned from the images alone and will be examined using PIV in a later section.

Figure 4.4 presents the recorded air pressure as a function of time corresponding to the experiment in Figure 4.2. Initially, the pressure increases at a constant rate as the air is compressed by the steady pump rate $\left(0.01 \mathrm{ml} \cdot \mathrm{min}^{-1}\right)$. Eventually the pressure reaches a required threshold ( $1700 \pm 50 \mathrm{~Pa}$ on average) to penetrate the water-grain packing, marked by a pressure drop as the air expands to fill the growing fracture. Once the pressure stored in the invading gas drops low enough that the friction in the granular packing can hold it back ( $1600 \pm 50 \mathrm{~Pa}$ on average), the fracture growth stops and the pressure begins to build again. Note that the first peak is larger than the subsequent saw-tooth pattern because of the influence of the inlet during the filling process. This kind of irregular saw-tooth pressure evolution is characteristic of stick-slip frictional processes, indicating that it is the grain-grain and grain-cell friction that holds back the invading gas until the threshold pressure is reached. 
It is also important to note that unlike viscous fingering, the growth is not directional or channelled into dominant pathways but instead appears random in its orientation and selection of nucleation point. However, the fracture branches do tend to grow together to fill the local space around previously made branches. It was observed that one fracture event would often trigger events in other local branches, likely as the result of a flowinduced disturbance in the packing caused by the initial growth

\subsubsection{Growth of a Single Fracture}

As air invades the water-saturated granular packing, capillary forces at the interface impose a force on the grains, eventually overcoming the opposing friction from the granular bed. The air-water interface pushes the grains aside to form an air-filled cavity in the shape of a fracture. A close-up image of a section of a fracture length is shown in Figure 4.5. The fracture shape is irregular and the width of the fracture space varies. The widest sections measure around 5-10 bead diameters $(1-3 \mathrm{~mm})$, while at the narrowest, the fracture aperture is indistinguishable from the surrounding granular packing.

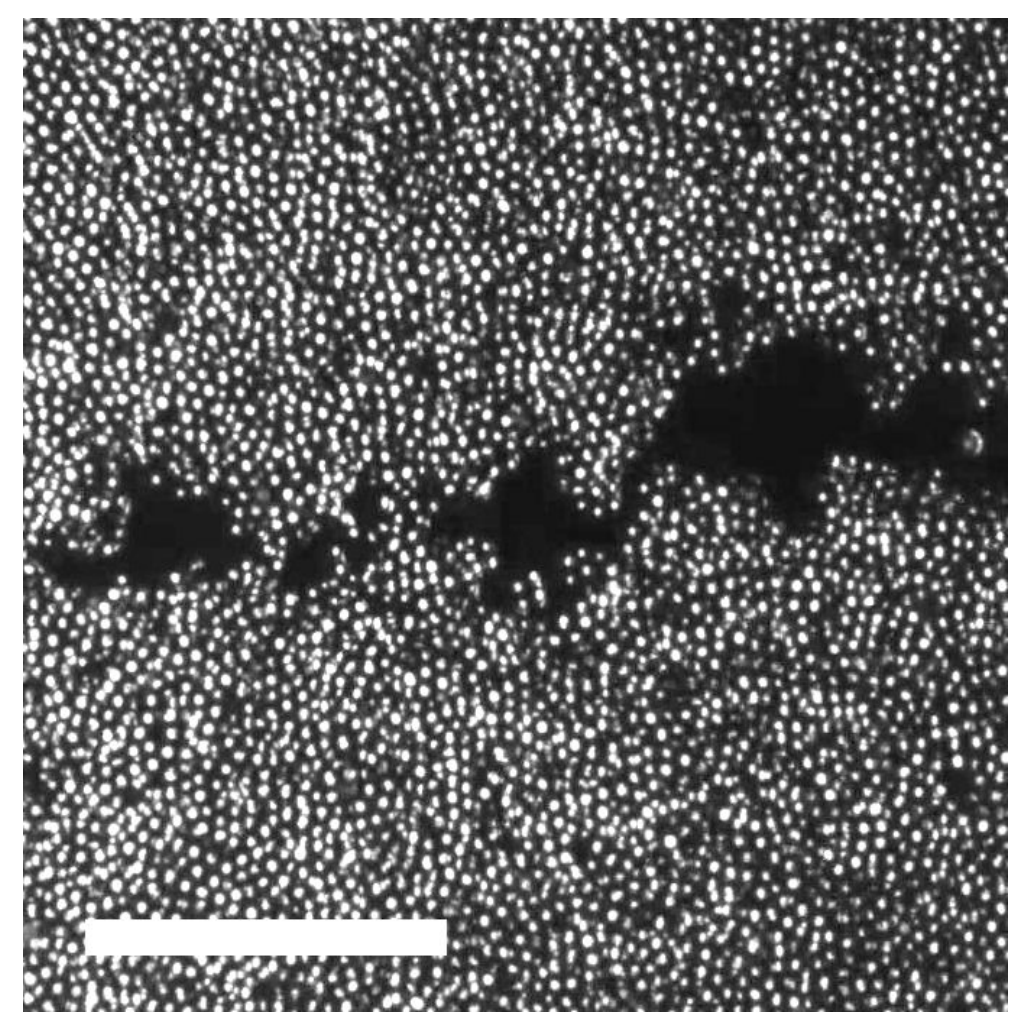

Figure 4.5: Close-up image of a gas-filled fracture cavity in a water-saturated granular packing in the small Hele-Shaw cell. Scale bar: $5 \mathrm{~mm}$. 

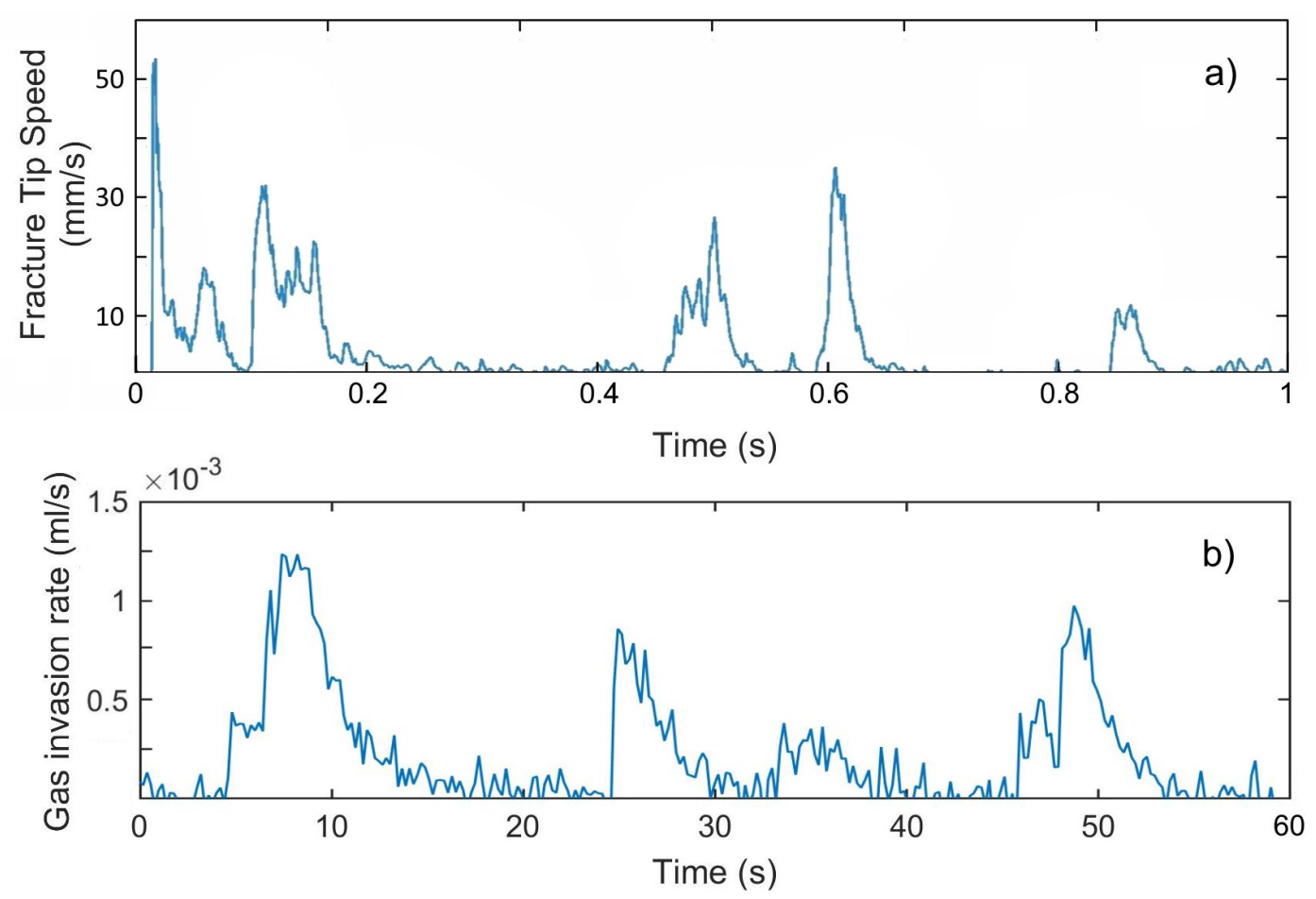

Figure 4.6: Intermittent motion at different scales. a) Shows the speed of the fracture tip during a fracturing event that lasted for $1 \mathrm{~s}$. b) Shows a section (minute 19) of the gas invasion rate for Figure 4.4 for a period of 1 minute.

Figure 4.6 (a) examines the speed of the fracture tip, calculated with PIV data from high-speed images, as it penetrates the granular packing in the smaller cell during a single recorded fracturing event that lasted for approximately $1 \mathrm{~s}$. For more than $10 \mathrm{~s}$ before and after the event there was no granular motion observed anywhere in the cell. The highest measured tip speed during the event was recorded for its initial motion at $54 \pm 0.5 \mathrm{~mm} . \mathrm{s}^{-1}$ for approximately $0.01 \mathrm{~s}$, the next three bursts of motion all had peak speeds of around $30 \pm 10 \mathrm{~mm} . \mathrm{s}^{-1}$, lasting approximately $0.06,0.05$, and $0.03 \mathrm{~s}$, while the final period of motion displayed a lower tip speed of $11 \pm 0.5 \mathrm{~mm} . \mathrm{s}^{-1}$ for approximately $0.03 \mathrm{~s}$. This evidences that there is intermittency in the granular behaviour of fracture growth, with five distinct periods of motion separated by brief pauses during one event, where one event is equivalent to one pressure drop in Figure 4.4. This intermittent behaviour was observed in all the recorded fracturing events. 
Note that there are two scales of intermittency at work here. The first, is in the larger scale stick-slip nature of the fracture pattern formation demonstrated by the saw-tooth pressure curve in Figure 4.4. Fracture growth is signalled by sharp drops in pressure due to air expansion, and over a 20 minute period there are approximately 48 large and small pressure drops that can clearly be distinguished, giving an average time of $25 \pm 5 \mathrm{~s}$ between these main fracture events. The second scale of intermittency is in the stick-slip nature of the granular motion within individual fracturing events captured by the highspeed camera and exemplified in Figure 4.6 (a), with five individual periods of motion taking place in the space of about $1 \mathrm{~s}$.

From the pressure signal recorded using the large Hele-Shaw cell we can infer the rate of gas invasion into the granular-liquid mixture. Using Boyle's law $P_{0} \Delta V \approx-V_{\text {gas }} \Delta P$, where $P_{0}$ is ambient pressure, and we have assumed small volume and pressure changes, $\Delta V$ and $\Delta P$ respectively, during fracturing as well as ideal gas behaviour at constant temperature, we have a volumetric gas invasion rate during fracture growth:

$$
Q_{\mathrm{gas}}=\frac{\Delta V}{\Delta t}=-\frac{\Delta P}{\Delta t} \frac{V_{\mathrm{gas}}}{P_{0}}+Q_{\mathrm{pump}} .
$$

Figure 4.6 (b) shows $Q_{\text {gas }}$ plotted as a function of time over a one minute time duration where each fracture event recorded with low time resolution in the large Hele-Shaw cell appears as an individual peak. The high-speed camera results in Figure 4.6 (a) demonstrate that what appears as one single, large-scale, fracture event is in fact composed of several smaller stick-slip motions which are separated by fractions of a second.

Given that each rise and fall of the gas invasion rate in Figure 4.6 (b) corresponds to one fracturing event in the larger cell, it could be expected that the total length of time for a fracturing event in the smaller cell would be similar. However, the events in Figure 4.6 (b) last from 3 to $10 \mathrm{~s}$, compared with $1 \mathrm{~s}$ for the longest recorded event in the smaller cell as seen in Figure 4.6 (a). There are a couple of explanations for this discrepancy. First is that, as mentioned previously, fracturing events in the larger cell often trigger more events in other branches, causing the period of fracturing to last longer. The smaller cell was made to specifically minimise this effect. Second is that when the invading gas was not fracturing it would frequently evacuate water from pore spaces around fracture branches via capillary invasion, i.e. without any motion in the grains. These small invasions likely also occur before, during, and after fracture growth, increasing the time duration of the fracture events as inferred from the pressure data, as well as resulting in minor instances of invasion between fracture events, explaining the minor peaks in Figure 4.6 (b) between the fracturing events. However, the meniscus of the water in the packing (the interface between water and the advancing gas) could not be seen in the high-speed imaging, and so all of the PIV analysis presented is representative of the granular motion only, with no capillary invasion being observable or recorded in our data. 


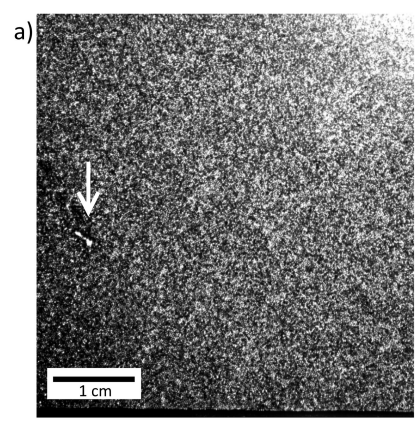

d)

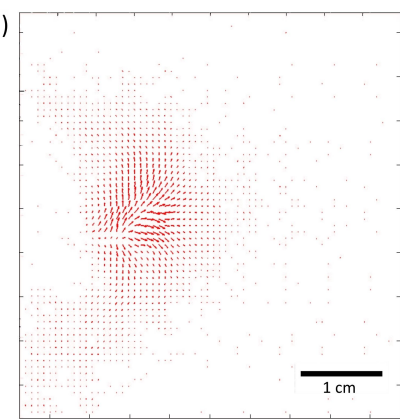

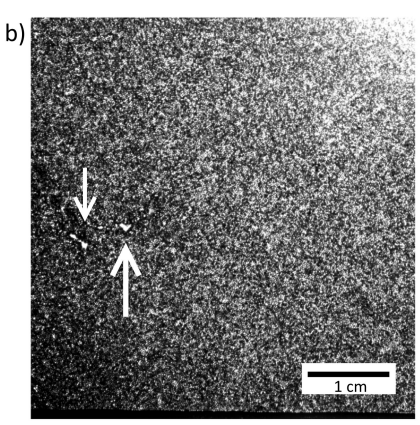

e)

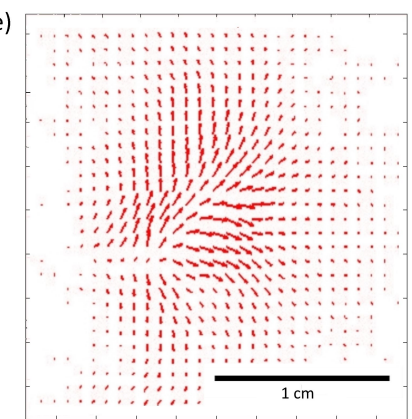

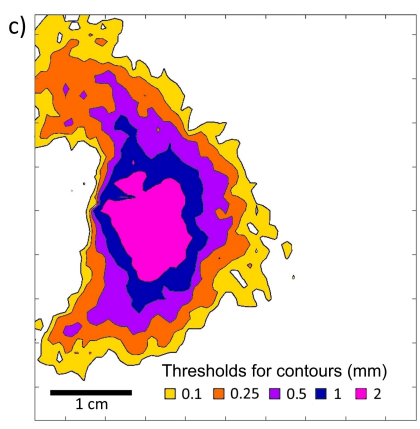

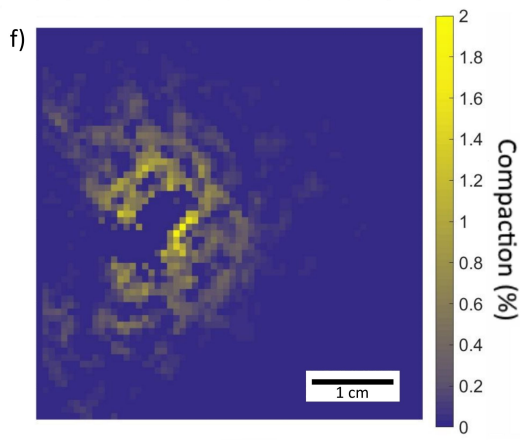

Figure 4.7: Example of granular motion during fracturing. a) Raw video frame before a fracturing event takes place. b) Raw video frame after a fracture event that lasted $0.13 \mathrm{~s}$ (initial arrow points to original fracture cavity, while the second points to the new fracture cavity). c) Contour plot of displacement field for grains over the fracturing event. d) Velocity vectors for (c) showing the direction of granular motion.

e) Zoom in of plot of (d) on the fracture cavity. f) Compaction relative to initial packing fraction as a result of the deformation caused by grain displacement field during fracturing.

\subsubsection{Deformation of the Granular Bed}

We studied the granular motion during fracture growth by determining the displacement profiles of the grains as the invading gas deformed the packing. For example, Figure 4.7 (a) shows the raw image of the packing before a fracturing event while Figure 4.7 (b) shows it after. The fractures are rather small at this scale, however looking at the overall displacement contour profile for this event in Figure 4.7 (c) reveals that the deformation caused is considerably wider than the fracture itself. Some of the minimally disturbed grains, moving at least $0.1 \mathrm{~mm}$, are up to $26 \mathrm{~mm}$ away from the parent fracture, and the region of the most disturbed grains surrounding the fracture, moving at least $2 \mathrm{~mm}$, has a radius of approximately $8 \mathrm{~mm}$ compared to the widest point of fracture width of $0.7 \mathrm{~mm}$. Though the influence of the fracture growth is pronounced on a surrounding area that is at least an order of magnitude larger than the fracture width itself, it quickly drops off and remains local to the vicinity of the active fracture. 
The compaction relative to the undisturbed packing as a result of that fracture event is presented in Figure 4.7 (f) where the compaction is found to peak at $2 \pm 0.5 \%$ and average at $0.5 \pm 0.5 \%$. For reference, the compaction of a random loose packing of spheres by $2-5 \%$ is equivalent to a bed being vibrated into close packing [176]. The shape of the compacted area mimics the shape of the displacement profile, compacting most significantly on the sides and in front of the fracture path and then significantly dropping off further away from the fracture. The resolution of the sub windows in the compaction PIV data is insufficient to resolve the width of the gas filled fracture, leading to the exaggerated size of the fracture width.

In both the displacement and compaction profiles it can be seen that the granular disturbance is avoiding the region behind the fracture's origin. This area holds the previous fracture branch (which can be seen in Figure 4.7(a)), as well as its previously compacted material. The compaction of the granular material causes narrowing of pore throats, leading to increased capillary invasion pressures along the fracture interface. The compacted front also has a high frictional resistance to motion due to stress dispersion to the confining plates. This is why subsequent granular motion avoids these zones and the gas invasion initially proceeds through pore spaces without mobilising grains. The invading gas needs to escape the compacted zone around the parent fracture cavity before it can create a new cavity where grains are still free to be moved.

To obtain a broader picture of this granular motion, the PIV data of 783 images of active fracture growth from multiple fracturing events were processed and averaged to form the velocity profile presented in Figure 4.8. The overall profile illustrates that granular motion is mostly in the direction of growth and to either side of its point of nucleation in the centre of the figure. The notable valley, or pinch in the profile behind the point of nucleation is the result of the previous fractures influence on the packing preventing motion. The majority of velocity profile is a halo of minor movement (less than $4 \mathrm{~mm} . \mathrm{s}^{-1}$ ) surrounding the nucleation point approximately $5 \mathrm{~mm}$ in all directions. The centre of the profile shows the average maximum velocity of the grains to be $20 \pm 5 \mathrm{~mm} . \mathrm{s}^{-1}$. This velocity is relatively rapid for grains of radius $0.2 \mathrm{~mm}$, but the individual grains move for approximately $0.1 \mathrm{~s}$ as the invading gas front advances past them, resulting in a displacement of only around $2 \mathrm{~mm}$ (see Figure 4.7). Away from the nucleation point the velocity of the grains rapidly decreases as the motion is dispersed among the granular material. This dispersal of motion is only possible because the grains begin in a random loose packing, with room to be compacted further. 

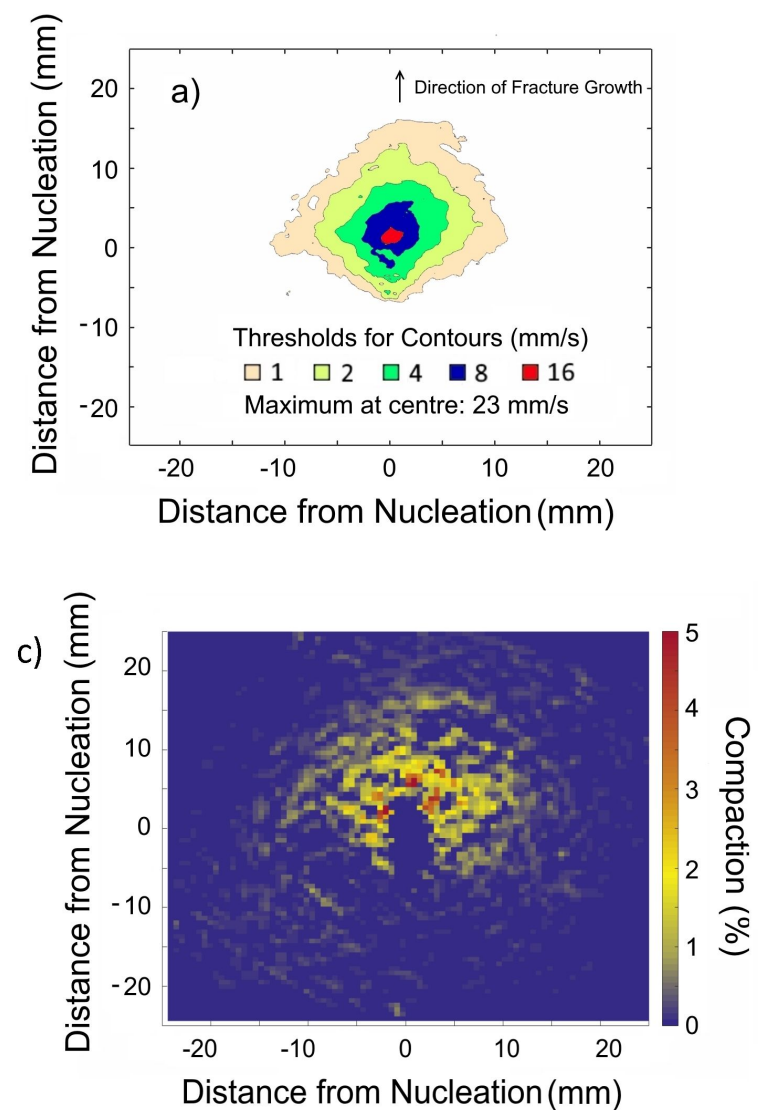
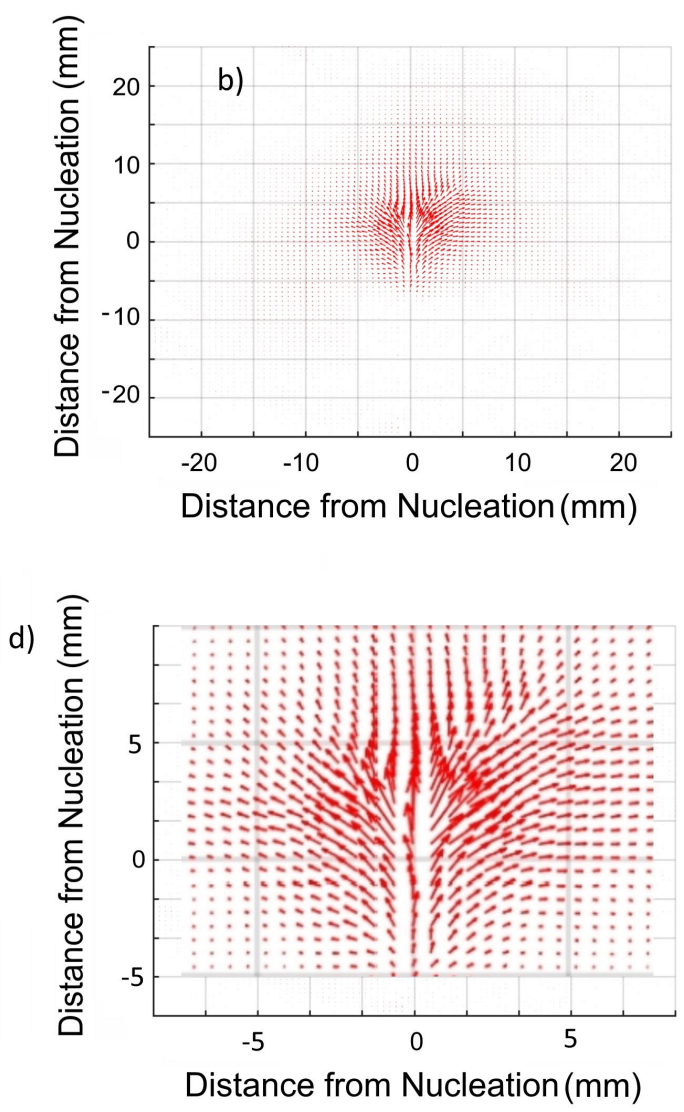

Figure 4.8: Average granular motion. a) Average velocity profile of grains with respect to distance from the point of nucleation during fracture growth, calculated using 783 PIV fields from $1 \mathrm{~ms}$ video frames of multiple fracture events. b) Velocity vectors for (a) showing the direction of granular motion during fracturing. c) Average compaction profile of grains caused by fracture growth with respect to distance from the point of nucleation calculated using Equation 4.1. d) Zoom in of plot of (b) on the nucleation point.

\subsubsection{Compaction Fronts as a Constraint on Pattern Formation}

The air pressure applies a stress on the interfacial grains that disperses through grain-grain contacts in the compacted region surrounding the fracture. Some of the granular stress is redirected to the confining wall of the cell, increasing the frictional resistance needed to be overcome to expand the fracture. A new fracture will expand to a maximum width such that the friction from the compacted zone balances the air pressure at the fracture wall. The compaction data calculated from the PIV data is displayed in Figure 4.8 and reveals a halo of compaction around the fracture nucleation point in the direction of its growth. 


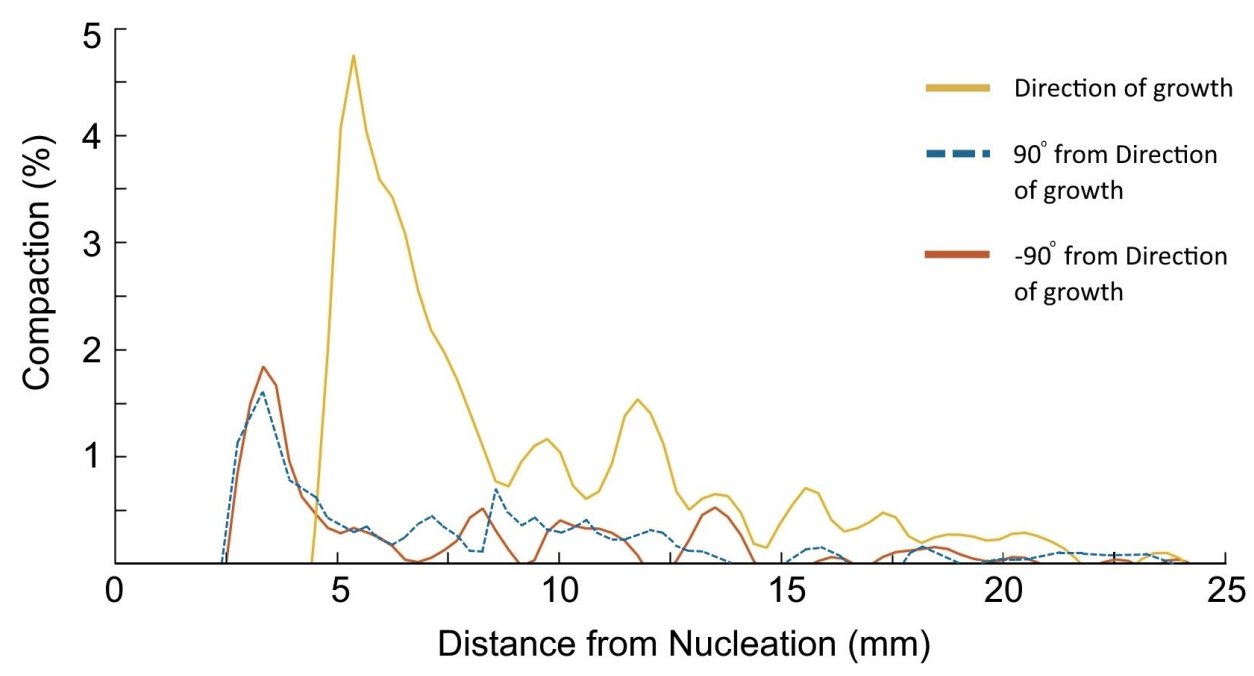

Figure 4.9: Compaction of the granular packing as a function of distance from the fracture nucleation point in the direction of growth (solid line with largest peak), $90^{\circ}$ from the direction of growth (dashed line), and $-90^{\circ}$ from the direction of growth (other solid line).

Figure 4.9 displays the percentage of compaction relative to the undisturbed packing as a function of distance from the point of nucleation in the direction of growth (yellow), $90^{\circ}$ from the direction of growth (blue), and $-90^{\circ}$ from the direction of growth (red). Here it can be seen that either side and in front of the fracture nucleation point are regions of marked increase in compaction. The peak of the compaction zones on either side are 1.5 and $2.0 \pm 0.5 \%$ with both occurring at $3.4 \mathrm{~mm}$ from the point of fracture growth, and each rapidly fall to half of their peak at approximately $4 \mathrm{~mm}$ from the origin. The compaction zone in the direction of growth is notably larger with a peak of $5.0 \pm 0.5 \%$ occurring at $5.4 \mathrm{~mm}$ from the point of fracture growth, and falls to half its peak value at approximately $7 \mathrm{~mm}$ from the origin. The larger compaction in the direction of growth is not surprising, and can help explain the branching meandering path the fractures take through the packing since the grains to either side are always more free to move than those directly ahead.

The formation of a compaction zone around fracture growth explains two notable phenomena in three-phase fracturing. First, the narrow pore sized necks that are observed before each fracture cavity imply that the capillary entry pressure must be lower than the pressure required to mobilise the compaction zone; however, the uncompacted packing beyond the compaction zone can be mobilised by that pressure as the invasion quickly expands into a new fracture cavity. This competition between air pressure and frictional forces will be discussed further in Chapter 4. Second, with each fracture branch having a compaction zone around it, the structure of the pattern in larger Hele-Shaw geometries will self-avoid. The fractures cannot grow into already compacted regions, and seek out undisturbed material. 
If we imagine two fractures that have grown parallel to each other as close as possible, they will be separated by a distance equal to each of their compaction fronts aligned on that side and half of each of their fracture widths, as illustrated in Figure 4.10. Simply put, the width of space occupied by a fracture and its compaction fronts, that no other fracture can then approach is:

$$
O_{w}=W+2 L
$$

where $O_{w}$ is the total occupied width of a fracture and its fronts, $W$ is the fracture's internal width, and $L$ is the width of a compaction front. Using the point at which compaction drops to below half of its peak as the boundary of the compaction zone, with Figure 4.9 we can determine that $0.5 \mathrm{~W}+L$ is on average $4 \pm 0.5 \mathrm{~mm}$ for these experiments, giving an estimated $O_{w}$ value of $8 \mathrm{~mm}$. As such, we would expect that a fracture pattern in this system would always form with fractures at a minimum of that distance apart. Fracture branches might grow further away from each other than that, but they can grow no closer.

In the larger cell we see that the invading gas seeks out undisturbed regions of the packing, producing a pattern that tends to locally fill the space of the system, with branches being separated by this $O_{w}$ length scale. For example, the average distance between fractures in Figure 4.2 (d) and other patterns made in the same system is $9 \pm 0.5 \mathrm{~mm}$. This is in good agreement with our calculated estimate of $8 \mathrm{~mm}$ and is likely larger because the fractures will not all conveniently grow parallel to each other. Another useful way to describe this phenomena is that, given the gas-driven fractures will eventually grow to space-fill a system, the inverse of the $O_{w}$ value (in units of $\mathrm{cm}$ ) is the average number of fractures encountered when moving $1 \mathrm{~cm}$ in any direction from a point in the completely fractured packing. Thus, an approximate spatial density of the fracture pattern can be defined as:

$$
D=\frac{1}{O_{w}}=\frac{1}{W+2 L},
$$

where $D$ is the spatial density of the fracture pattern. In this case the spatial density of the patterns is $1.1 \mathrm{~cm}^{-1}$, while our upper limit derived from the compaction front data is $1.3 \mathrm{~cm}^{-1}$.

\subsection{Conclusion}

We have used close-up, high-speed imaging to analyse the motion of cohesionless grains during the formation of fracture cavities as a result of a loose granular packing saturated with water being subjected to a forced invasion of air. The observed sub-millimetre and microsecond scale events were examined in the context of, and were compared to, the larger scale observation of fracture patterns in a Hele-Shaw cell that are defined by a characteristic length scale. 


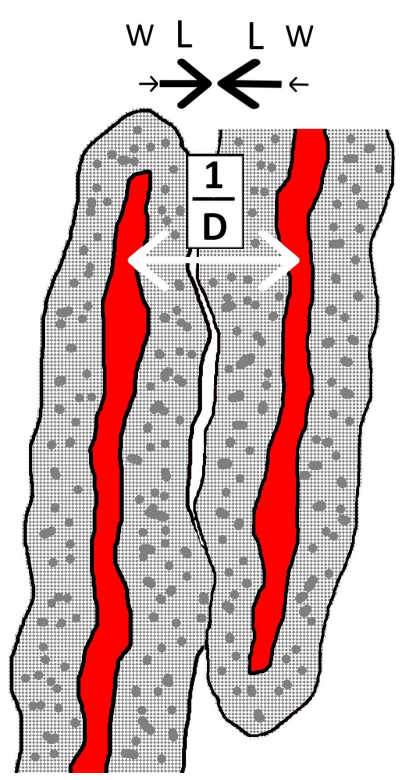

Figure 4.10: Illustration of two parallel fracture cavities (enclosed filled areas) and their touching compaction fronts (surrounding speckled areas). The distance made up from the fracture widths (W) and their compaction fronts (L) keeps the fractures a minimum set distance apart that is equivalent to the inverse of their spatial density (1/D).

In concordance with earlier studies [13, 21, 24, 70, 78, 178-180], a network of conductive pathways in the shape of fractures is observed. As the pressure in the system builds, the capillary and viscous forces eventually overcome frictional resistance of the grains and fractures are forced open, relieving the pressure in the invading gas. This process then repeats resulting in stick-slip intermittent growth. The fracture branches avoid each other when branching, but yet still remain relatively local as their nearby deformations in the packing stimulate local growth. 
Studying the growth of individual branches with high-speed video revealed other scales of intermittency during fracture growth. Fractures were seen to have frictional behaviour within fracturing events, with the fracture tip speed varying and pausing in its own stick-slip manner (Figure 4.6). The gas front was regularly observed to invade through pore spaces while the surrounding packing remained static, indicating that the immediate grains were resistant to motion while a fracture cavity could be formed in those found further along the invasion path. PIV analysis of the high-speed imaging of multiple fracturing events allowed us the generate an average velocity profile for fracture growth (Figure 4.8). This profile shows the wide influence of the deformation caused by fracturing in comparison to the size of the fracture. The shape of the velocity profile revealed regions behind and to either side of the point of growth that granular motion avoids as a result of deformation cause by the previous fracture. As such, a compaction profile was also generated, displaying the halo of compaction caused by fracture growth. This explains why the gas first invades through pores spaces before mobilising grains beyond its previous compaction front, why the fractures self-avoid in the larger scale pattern forming process, and allows us to calculate the upper limit for the spatial density for fracture patterns in these circumstances.

Geological phenomena such as sedimentary methane venting, magma degassing, and soil drying are known to incorporate processes involving gas-driven fracturing of saturated packings and granular suspensions, and as such our techniques and results can contribute to an improved understanding of their resultant fracture network properties and the threephase gas, liquid, and grain interactions that cause them [9, 11, 181-183]. Several authors have pointed to intermittency in the opening/closing of fractures as a characteristic feature of both methane vents in sediments [10, 59, 65, 69, 184] and gas conduits in magma [9. 82, 185]. Our results show that this system-scale intermittency is echoed between individual fracture events as well as within an individual fracture event.

In addition, being able to predict spatial density of fracture patterns in saturated granular material is particularly relevant in engineering applications such as stimulating hydrocarbon reservoirs and soil remediation where increasing the permeability of a bulk granular material with fracture networks can greatly increase efficiency of fluid exchange [14, 15, 186]. 
Overall, the techniques and results presented here illustrate that multiphase fracturing in saturated granular material is even more complex than previously thought, with relevant phenomena occurring across multiple length and time scales over different orders of magnitude. Future work can be directed into multiple avenues of questioning: is it possible to observe the motion of the water meniscus during fracturing events and would doing so help explain the branching process in the invasion pattern; can these fractures be studied in a fully 3D system and are the resultant invasion patterns also dominated by compaction fronts; how does changing the interstitial or invading fluids affect the dynamics observed here; and can varying the properties of the packing to make heterogeneous layers be used to model these processes in sedimentary systems. 


\section{Chapter 5}

\section{Parameters of Fracture Pattern Formation}

\subsection{Introduction}

The previous chapter established that it is the formation and presence of compaction fronts around fracture growth that explain many of the interesting aspects of gas driven fracturing in saturated granular material. The compaction fronts thicken until their frictional properties make it more favourable for the gas to invade the pore spaces between grains, rather than moving the grains themselves. This means that once a compaction front has been formed, no further fracturing can occur in that region; the pattern will self avoid and enter undisturbed areas. As such, the thickness of these compaction fronts is fundamental to the spatial density of the resultant pattern.

Being able to predict the spatial density of a fracture growth within a system of saturated granular material subject to the invasion of gas would be useful in many of the applications outlined in Chapters 1 and 3. This chapter focuses on understanding and illustrating how the physical properties of the system and the granular material influence these compaction fronts. We develop a model that predicts the front thickness, and therefore the spatial density of the pattern, based only on these physical properties. The model was tested, and shown to be accurately predictive with regards to grains size and frictional properties. Finally, we find that the model has a limit with respect to the injection rate of the gas, below which the model is independent of the rate and above which the invasion patterns become viscous and aligned with the fluid flow out of the system.

The experimental setup in this chapter was designed by James Campbell with the supervision of Bjørnar Sandnes. The experimental results presented in this chapter were performed and analysed by myself with the supervision of Bjørnar Sandnes, with the exception of section 5.3.6 which was performed and analysed by James Campbell also with the supervision of Bjornar Sandnes. 


\subsection{Materials and Methods}

\subsubsection{Experimental Setup}

Experiments were performed in the larger Hele-Shaw cell mentioned in the previous chapter, as shown in Figure 5.1 (a). Two $350 \times 350 \times 18 \mathrm{~mm}$ glass plates were held together by $0.5 \mathrm{~mm}$ adhesive plastic spacers; these sealed three sides of the cell, leaving a $200 \mathrm{~mm}$ wide channel which was open at one end. A hole was drilled through one of the plates providing an inlet at the closed end of this channel, $300 \mathrm{~mm}$ from the open end.

A bed of loosely packed glass grains was formed within the cell by turning it vertically, filling it with deionised water and pouring the grains into the top of the channel to sediment downwards. A head of water of about $1 \mathrm{~cm}$ was left between the top of the granular bed and the end of the cell to prevent evaporation from influencing results.

After the bed had fully settled, the cell was turned horizontal. Air was injected into the inlet at various rates using a syringe pump (Harvard Scientific, PHD Ultra), while a pressure sensor (Sensortechnics) logged the relative air pressure at $5 \mathrm{~Hz}$. No pressure log was taken at rates above $10 \mathrm{ml} \cdot \mathrm{min}^{-1}$ as the pressure rose above the range of our instrument. Injection was initially done at a slow rate $\left(0.03 \mathrm{ml} . \mathrm{min}^{-1}\right)$ to push through excess water in the inlet without disturbing the grain packing; afterwards (but before the appearance of the first fracture) the rate was raised or lowered to the rate of interest and held there for the duration of the experiment. Photographs were taken at regular intervals to record the results. A gas injection volume of $8 \mathrm{ml}$ was used for all experiments presented here. The edge of the channel was kept unobstructed (open boundary conditions) in all experiments.

Both spherical beads and rough, irregular grains of soda-lime glass were used as shown in Figure 5.1 (b) and (c), sieved to various size ranges between 53 and $250 \mu \mathrm{m}$. The bead beds had measured initial porosities $n_{0}$ between 0.415 and 0.465 (increasing with increasing bead size due to edge effects), consistent with values expected for a random loose packing of spheres [176]. Irregular grains, which were sieved to $75-100 \mu \mathrm{m}$, packed with a significantly higher porosity of $n_{0}=0.63$. Both beads and irregular grains were washed in a 3:1 mixture of sulphuric acid and hydrogen peroxide (piranha solution), and rinsed thoroughly in deionised water prior to use. The piranha solution treatment ensures removal of contamination and a highly wetting, hydrophilic surface. Piranha treated sodalime glass is estimated to have an air-water-glass contact angle of $\theta=12 \pm 5^{\circ}$ [175]. Bed porosity was determined by finding the mass of granular material needed to fill a known volume of the cell. Permeability was found by measuring the pressure difference needed to push water through the bed at $0.03 \mathrm{ml} \cdot \mathrm{min}^{-1}$, which relates to the permeability by Darcy's law [187]. 

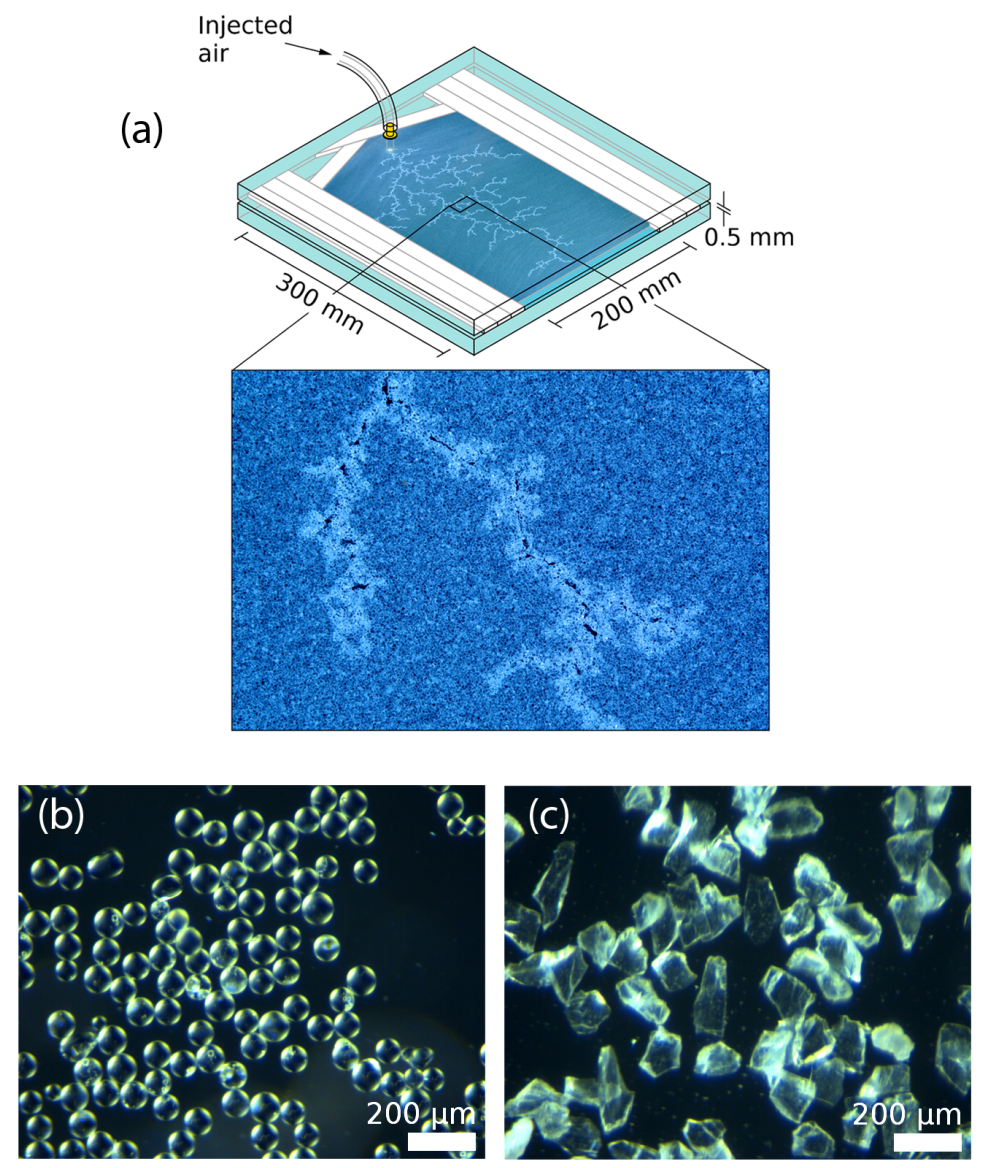

Figure 5.1: Experimental setup. (a) Through an inlet, air invades the water-saturated granular packing in a branching fracture pattern. (b) Spherical beads and (c) irregular grains used in the study, both sieved between 75 and $100 \mu \mathrm{m}$.

\subsubsection{Data and Image Analysis}

Fracture patterns were analysed at the moment they first reached $20 \mathrm{~cm}$ from the inlet to avoid edge effects. For comparisons between rates, $15 \mathrm{~cm}$ was instead used for the cut-off due to fluidisation of the granular packing close to the edge of the cell at high rates. Processing was performed using the same process described in the previous chapter (image subtraction, thresholding, despeckling, and a closing operation were applied in sequence to produce the binary images presented here). The total length of the fractures was found as half the perimeter of the pattern. To find the branching properties, the pattern was skeletonised after manually correcting any remaining gaps in the image. A custom algorithm (developed by James Campbell) then translated the skeletonised image into a logical branching structure, removing any detail on scales below $1 \mathrm{~mm}$. 
Fracture density was measured as the fracture pattern length divided by the area of the convex hull of the pattern. Mean cross-sectional area of the fractures was found by using the pressure log and known injection rate to infer the total volume of invaded gas, and dividing this by the total fracture length. The peak pressure is simply the highest gas pressure achieved during the course of the experiment. We define tortuosity as the ratio between the direct Cartesian distance to the inlet and the distance following the path of the fractures; the mean tortuosity is merely this averaged over the whole pattern.

To study the system dynamics in more detail, a smaller region was observed in the centre of the cell at higher magnification and at 60 frames per second. Particle Image Velocimetry (PIV) was performed on the images using the OpenPIV software package.

\subsection{Results and Discussion}

\subsubsection{Fracture Growth in the Frictional Regime at Low Injection Rate}

This section describes the dynamics of fracture growth at injection rates of $0.1 \mathrm{ml} / \mathrm{min}$ and below. A later section discusses the rate dependence of the process.

Figure 5.2 (a) shows a time series of typical fracture growth for 53-100 $\mu \mathrm{m}$ spherical beads. The injected air invades the granular bed by opening a branching pattern of narrow fractures, typically never more than three bead diameters wide. The pattern has an apparently random bifurcating structure with branches opening by a process of tip-splitting. The fractures never intersect each other. Figure 5.2 (b) shows the growth dynamics for a full fracture pattern coloured according to formation time. Fractures are sometimes seen to grow preferentially along the edge of the packing. This is likely an effect of locally inefficient packing close to the walls.

Figure 5.2 (c) shows the variation in gas pressure alongside the fracture growth rate over the course of a typical experiment. Although the gas is injected at a constant rate, the fracturing is intermittent, with periods of inactivity during which the pressure builds, and short bursts of rapid growth. Such behaviour is common in friction-dominated systems such as stick-slip sliding [188], earthquakes [189] and granular avalanches [190]. It is to be supposed that grain-grain and grain-cell friction hold back fracture expansion until a threshold pressure is achieved, at which point there is rapid growth curtailed by a consequent pressure drop. Up to $0.1 \mathrm{ml} / \mathrm{min}$, the gas injection rate has little or no impact on the magnitude of these slip events but rather determines the length of the stick periods between slips, as demonstrated in Figure 5.3 . 
(a)

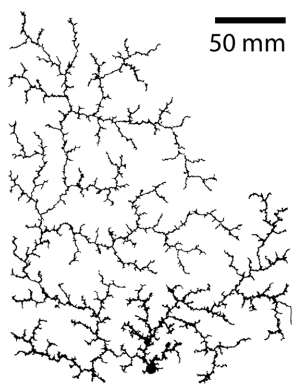

(b)

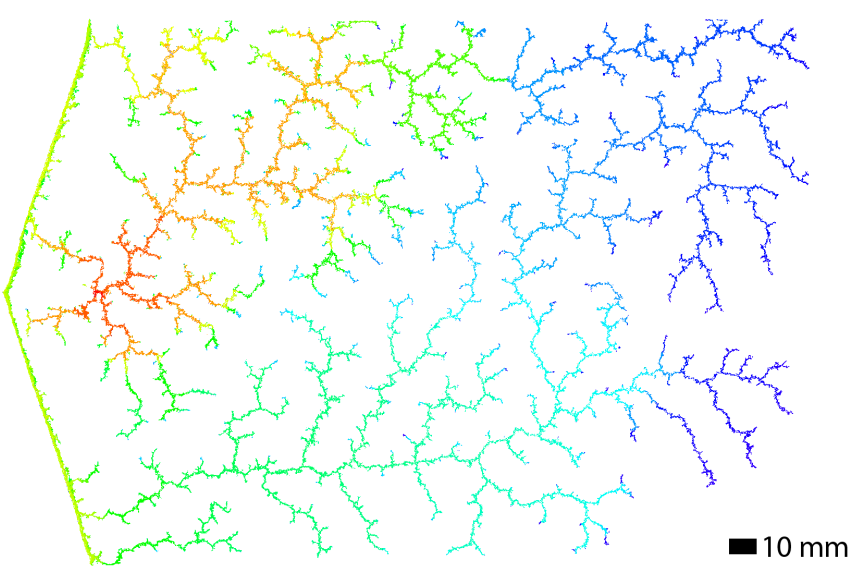

(c)



Figure 5.2: Fracture growth. (a) Thresholded images of fracture pattern at $\Delta t=350 \mathrm{~s}$ time intervals. (b) Fracture pattern coloured by formation time. (c) Gas pressure (black line) alongside fracture growth rate derived from image sequence (coloured bars), showing stick-slip behaviour characterised by short bursts of rapid growth. Colour scales in (b) and (c) are identical. (Injection rate $q=0.03 \mathrm{ml} / \mathrm{min}, 53-100 \mu \mathrm{m}$ beads.)

Figures 5.4 (a)-(c) look in more detail at the dynamics of a single slip event. One sudden drop in pressure is often seen to be the result of the almost simultaneous growth of several lengths of fracture, usually local but disconnected from one another. We conclude that a single slip event causes a chain reaction in neighbouring regions that are close to their threshold pressure, caused by fluid flow induced disturbance of the packing in the vicinity of a growing fracture. Figure 5.4 (c) shows that the seven active branches nucleated in sequence within a $0.5 \mathrm{~s}$ time window during this particular slip event. Fracture growth comes to a halt after 1-2 s. 

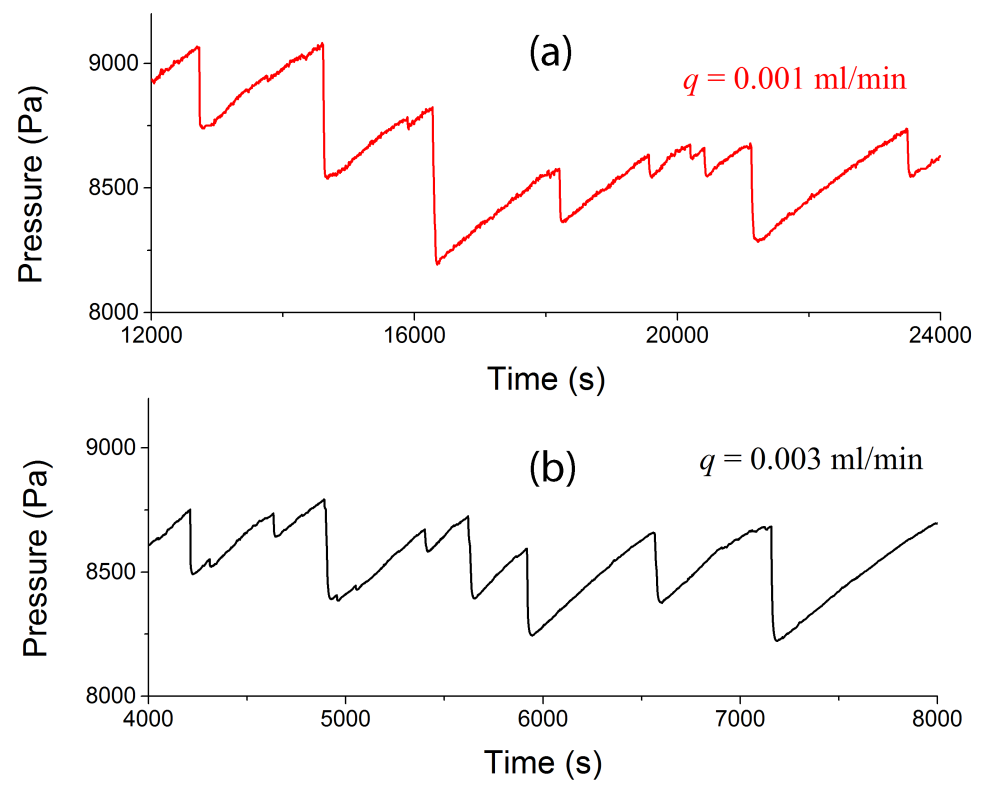

Figure 5.3: Gas pressure during stick slip fracturing for (a) $0.001 \mathrm{ml} / \mathrm{min}$, and (b) $0.003 \mathrm{ml} / \mathrm{min}$. The time axis shows a three times longer duration for (a) compared to (b).

\subsubsection{Granular Compaction Fronts}

Particle Image Velocimetry (PIV) analysis was performed on growing fractures and an illustrative result is presented in Figure 5.5. The fracture pushes grains ahead of it and to either side, creating a compacted front beyond which the packing is unperturbed. An applied force to a thick compacted front will be propagated to the out-of-plane confining walls via force chains within the material [191, 192], causing it to jam [193-195] and stop the fracture from expanding. Such compaction fronts have been observed before in frictional fingering experiments where the Hele-Shaw cell is only partially filled with granular material [20, 21]. Chevalier et al. studied fracturing of density matched granular suspensions, and found that the mobilization of grains occurred in the local vicinity of the advancing fracture tip for suspensions with high granular volume fraction [71].

Figures 5.6 (a)-(c) show an individual fracture with associated grain displacement field and granular bed density relative to the initial packing density. The analysis reveals the local compaction zone (red halo) surrounding the fracture. The resolution of the PIV data is insufficient to resolve the narrow width of the gas filled fracture, producing an exaggerated decompaction zone (blue colour). Figure 5.6 (d) shows the percentage compactivity measured as a function of distance to the nearest fracture branch, averaged over three separate fracture events. The local compaction of the granular material absorbs the deformation caused by the growing fracture. Thus, a key requirement for fracturing of a deformable porous medium is compactibility. We define compaction $\Xi$ as

$$
\Xi=\frac{n_{0}-n_{c}}{1-n_{0}}
$$




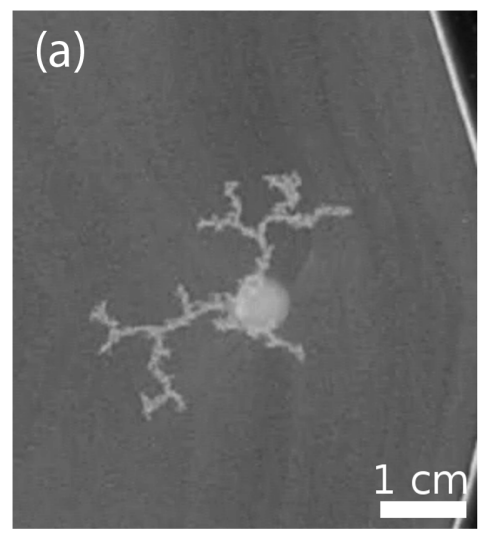

$\mathrm{t}=0 \mathrm{~s}$

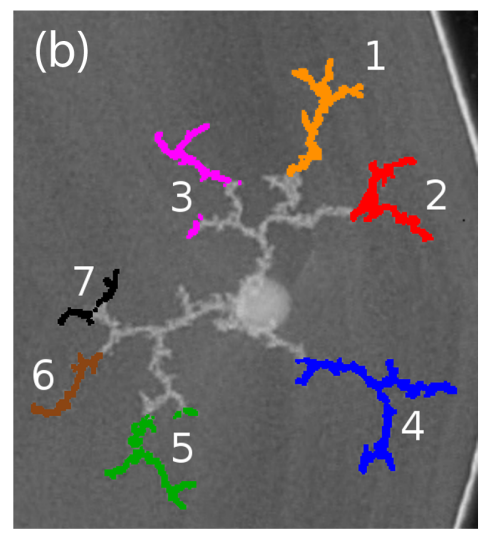

$\mathrm{t}=5 \mathrm{~s}$

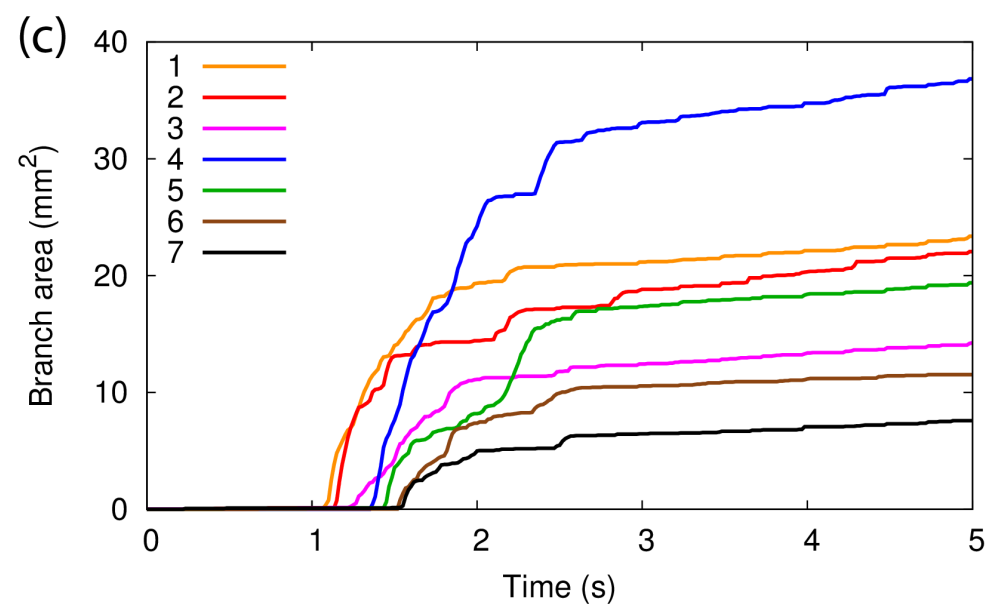

Figure 5.4: (a) and (b) show images of a fracture pattern five seconds apart during $0.01 \mathrm{ml} / \mathrm{min}$ injection. The growth area is divided into seven disconnected regions and labelled. (c) Variation of area of each region with time between above images, from analysis of video footage, showing synchronized growth after a prolonged stationary period.

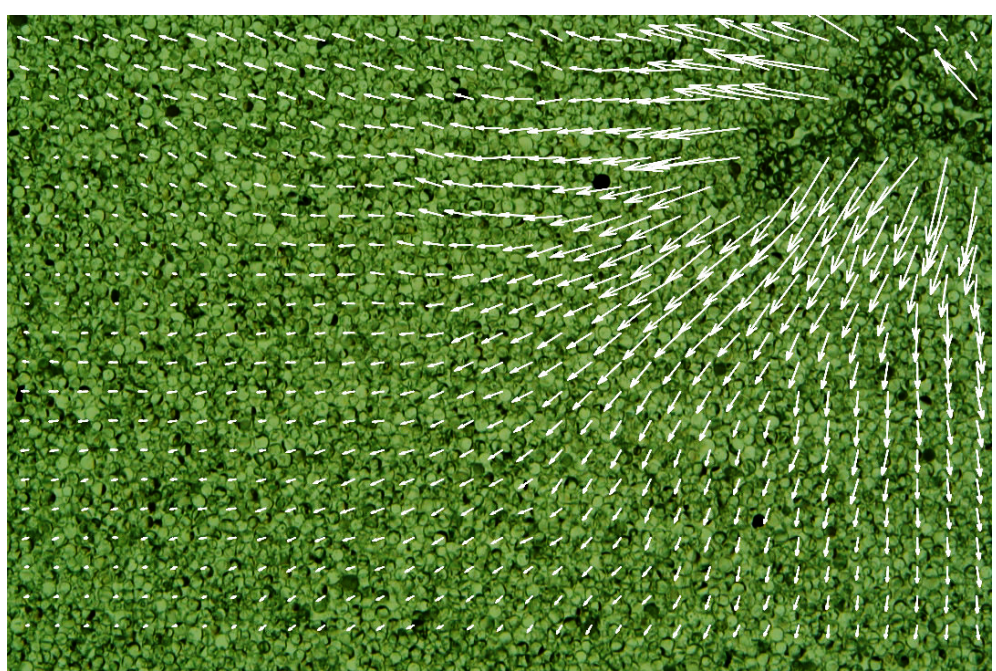

Figure 5.5: Close-up during growth of a fracture from the top right corner. Granular displacement vectors from PIV analysis show large deformation close to the growing fracture. Bead diameter: 150-200 $\mu \mathrm{m}$. 


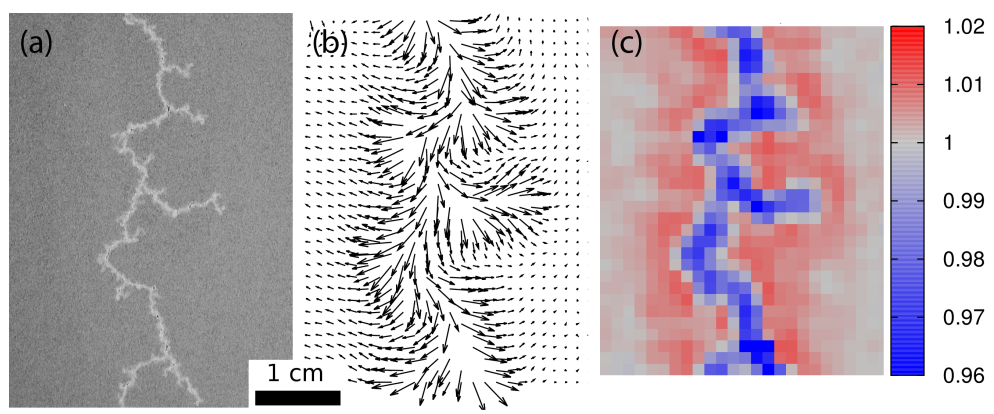

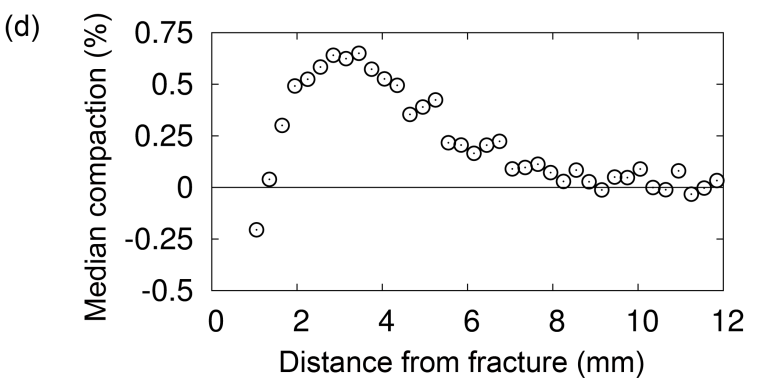

Figure 5.6: Compaction fronts. (a) Photograph of a fracture in 53-100 $\mu \mathrm{m}$ beads which propagated from top to bottom of the image, $0.1 \mathrm{ml} / \mathrm{min}$. (b) Grain displacement field, exaggerated $50 \times$. (c) Granular bed density relative to that at start of experiment, found from the divergence of the displacement field. Red areas show compaction, blue areas expansion. (d) Compaction data from three images such as (c) was binned by distance to the nearest fracture; this graph presents the median compaction as function of distance from fracture.

where $n_{c}$ and $n_{0}$ are the compacted and uncompacted granular bed porosities respectively. From Figure 5.6 (d) we can make a rough estimate of $\Xi \approx 0.7 \pm 0.1 \%$ as the peak value of the median compaction for 53-100 $\mu$ m beads.

As discussed in Chapter 4.3.4 in this thesis, the existence of these compaction fronts explains the self-avoiding nature of the fracture patterns: fractures cannot grow into the already compacted regions around existing fractures. The total cross-sectional width occupied by a fracture comprises the width $W$ of the (gas-filled) fracture itself and the compaction fronts either side, each of a thickness $L$. The length $W+2 L$ thus sets a lower limit for the spacing between separate, parallel fracture branches. Fractures may not necessarily approach one another as close as this, but they may approach no closer.

Observing the fully developed fracture patterns displayed in Figure 5.2, the patterns appear to fill the available space surprisingly efficiently for a random branching structure. The patterns appear fractal on small scale, but on the scale of the cell, a characteristic separation length between the fracture branches can be discerned. The pattern is more "space filling" than "fractal", and there is in Figure 5.2 never more than about a centimetre separating the bulk of the porous medium from the nearest fracture branch. 
The emergence of a characteristic length implies that the system displays a welldefined spatial fracture density $D$. Which we defined previously as:

$$
D=\frac{1}{W+2 L}
$$

Conceptually, $D$ is equivalent to the spatial frequency of straight, parallel fractures separated by two compaction fronts, i.e. maximally efficient invasion of the granular bed. If the compaction (Eq. 1) of the porous material is known, then the fracture width may be expressed as $W=2 \Xi L$, such that $D=[2(1+\Xi) L]^{-1}$.

This minimalist model will in a later section be developed to provide a prediction of fracture density from basic material properties, but for now consider Figure 5.6(d) which gives a rough estimate of the distance at which compaction drops below $50 \%$ of its maximum value as $5.5 \pm 0.1 \mathrm{~mm}$ for $53-100 \mu \mathrm{m}$ beads. This implies a minimum separation distance of $W+2 L=11 \mathrm{~mm}$, i.e. a fracture density of $D \approx 0.9 \pm 0.1 \mathrm{~cm}^{-1}$, which is in good agreement with measured values of $\sim 1 \mathrm{~cm}^{-1}$. (Experimental measurements of $D$ will be presented in more detail in a later section).

It can be imagined that in a confined, closed system fractures will eventually grow into unprocessed regions until all the available space is either occupied by fractures, their corresponding fracture fronts, or spaces too small to hold a fracture and its fronts. As such, closed systems would be expected to display fully fractured beds with well-defined fracture densities.

However, the setup we have used has an open boundary so the reason why the fracture network grows to fill space, rather than produce the fractal structures typically seen in e.g. viscous/capillary fingering in porous media, is not immediately obvious. It is possible that the partial confinement (channel geometry) may enhance the space-filling tendency compared to previous studies of fracturing from a central inlet where the fractures can spread out laterally in all directions [9, 24, 196]. Also, buoyancy effects in vertical cells [11, 13, 197] and viscous effects at higher injection rate [71] may significantly affect the pattern formation.

The dynamics of fracturing could itself promote a space-filling pattern. Consider Figure 5.4, where disturbance from fracture growth in one branch is seen to trigger the nucleation of additional fractures nearby. It seems likely that this cooperative action has the effect of creating a "process zone" of multiple fracturing events in the locality of the initial fracture nucleation. Thus the active growth region becomes fully invaded by the concurrently growing fractures, producing a pattern that is space-filling on a local scale. Fractal growth may still exist, but at a larger scale than that observed in these experiments.

Note that the patterns showed considerable variability, with patches incompletely filled by fractures (see Figures 5.7 and 5.11). The space-filling nature of the pattern is however evident in the fractured regions of the cell. 


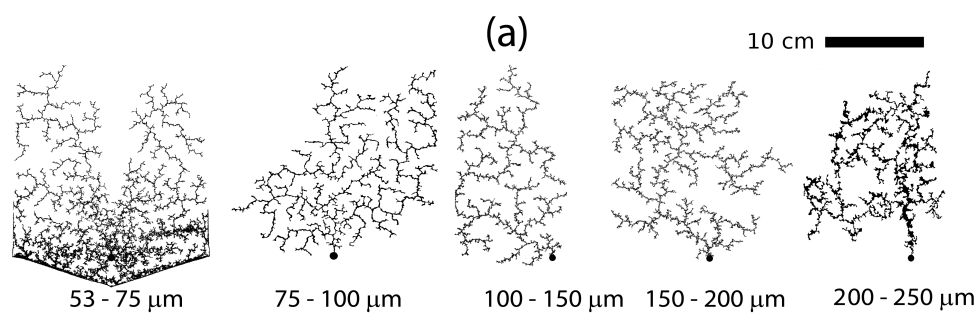

(b)

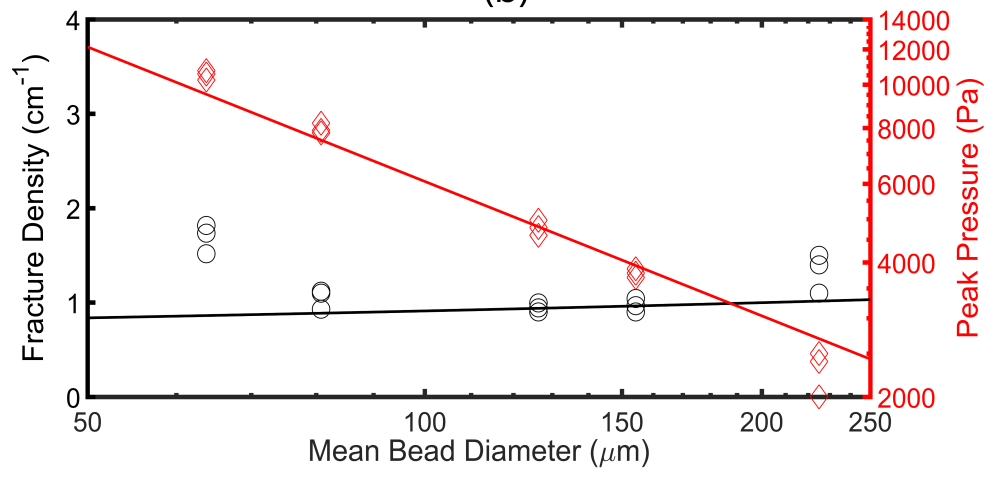

Figure 5.7: Effect of bead size. (a) Fracture patterns, and (b) fracture density (black circles) and peak pressure (red diamonds) as a function of measured mean bead diameter. Each symbol represents a single experiment. The black line shows the density predictions of Equation 5.7. The red line shows a fit of the peak pressure to a $d^{-1}$ dependency.

\subsubsection{Effect of Grain Size}

Figure 5.7 (a) shows fracture patterns for different size ranges of spherical beads. Although there is variability in the overall shape of the patterns, the fracture density in the fractured zones appears relatively insensitive to the grain size. The open circles in Figure 5.7 (b) depict the measured fracture density, and reveals an overall trend of a slightly increasing $D$ as a function of grain size. The exception is the lowest grain size (53$75 \mu \mathrm{m})$ where the experiment suffered from an artefact whereby the air invaded the space between the top glass plate and the granular bed, especially around the inlet. This effect is noticeable as dark patches in the lower part of the experiment shown in Figure 5.7 (a) (left).

While the pattern morphology appears relatively insensitive to grain size, this is not the case for the gas pressure at the point of fracturing which is observed to decrease with increasing grain size (Figure 5.7 (b) red diamonds). The red line is a fit to the Laplace pressure equation for the pressure difference $\delta P$ across a spherical interface [187]:

$$
\delta P=a \frac{4 \gamma \cos \theta}{d}
$$



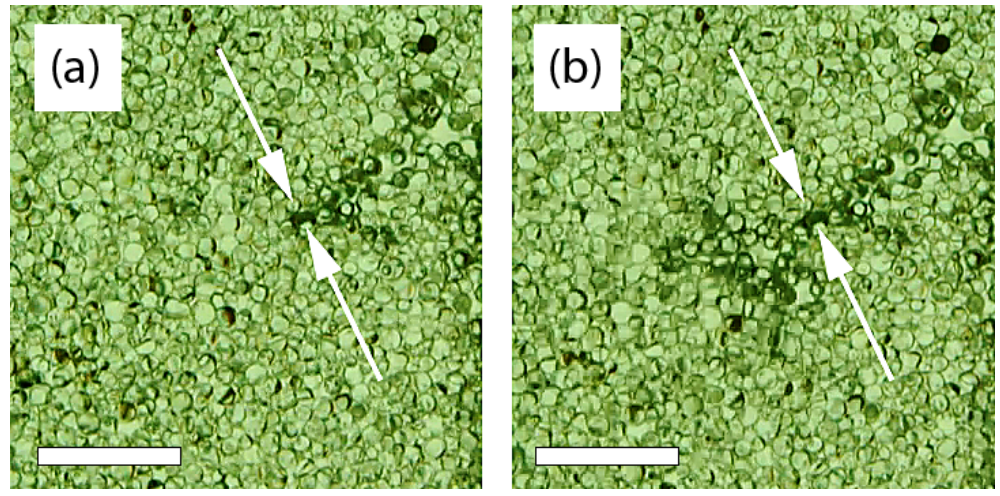

Figure 5.8: Onset of fracture growth. (a) and (b) show images taken at a $0.08 \mathrm{~s}$ time interval, with a new fracture starting to grow towards the lower left hand corner. Arrows point to stationary beads as the fracture nucleates by capillary invasion of the compacted front. Bead diameter: 150-200 $\mu \mathrm{m}$. Scale bar: $1 \mathrm{~mm}$

where $\gamma$ is the surface tension of water $(0.072 \mathrm{~N} / \mathrm{m}), d$ is the diameter of the beads, and $\theta$ is the contact angle, for which we used a value of $12^{\circ}$ [175]. $a$ is the free variable in the fit and is found to be 2.14 for the peak pressures as shown, with a lower value of approximately 2.0 for the average pressures at the onset of fracturing. Physically, this means that the measured fracturing pressure is equivalent to that needed to invade a hydrophilic cylindrical pore of diameter roughly half that of the beads.

Once a compaction front is established, it cannot simply be pushed out of the way by a higher pressure: this merely causes it to compact the grains behind it, thickening the front, as observed in simulation [49]; rather a fracture must somehow breach through the front. The pressure triggering the onset of fracturing corresponds to an interface curvature roughly 2.0 times that of the grains. For comparison, one study of pore throats in a random close packing of equal spheres (we assume that in the compaction front the grains are close packed) found the ratio between the maximum interface curvature and the sphere curvature to begin at 1.8 for the very widest pores in the system [198]. By this measure our fracturing pressure is close to the pressure at which we would expect capillary invasion into the largest available pores.

Capillary invasion between the grains in the static compaction front is thus the trigger for the nucleation of a new fracture, as observed in the previous chapter. The fluid flow from the capillary invasion is sufficient to briefly mobilise the packing, leading to a selfreinforcing process of invasion causing flow leading to bead mobilisation that triggers rapid invasion of air in the form of fractures. Figure 5.8 shows the point of nucleation of a new fracture branch. The beads in the initial compaction front remain largely undisturbed as the gas penetrates the front by pore invasion. As the invading gas emerges on the other side of the compaction front, the packing is mobilized, and the fracture expands by pushing grains ahead and to the sides. 

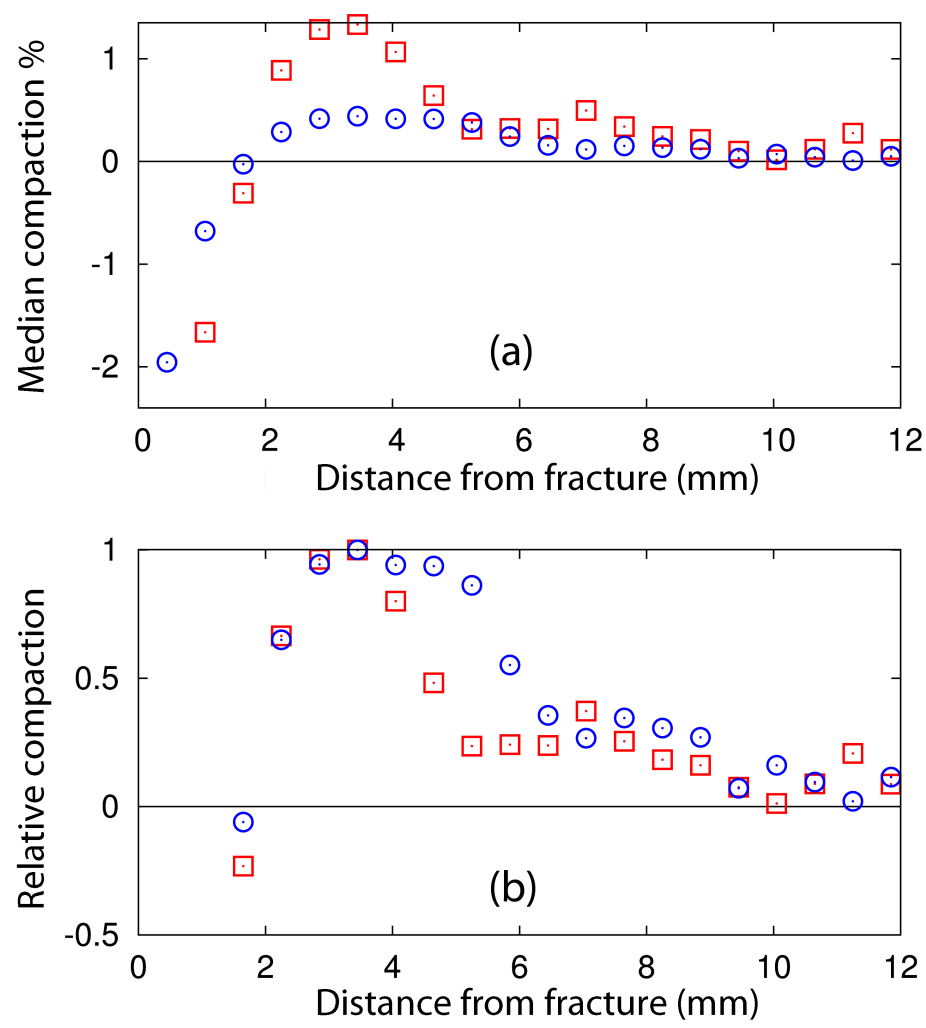

Figure 5.9: (a) Median compaction at each distance for $75-100 \mu \mathrm{m}$ spherical beads (blue circles) and irregular grains (red squares). (b) The same data normalised relative to the maximum compaction for each grain type, showing the varying widths of the compaction fronts.

The concept of capillary fracturing described above follows that of previous work by other authors [24, 184, 199], but for a subtle difference: the emergence of the compaction front requires the pressure to exceed the capillary pore entry pressure of the close-packed material in the front. It is not enough for the capillary forces to exceed the deformationor frictional resistance of the undisturbed medium. Instead there is a feedback effect where the thickness of the compaction front grows to balance the gas pressure, forcing the pressure to increase to the point of capillary invasion (without necessarily disturbing the grain configuration). Fracturing as observed in the experiments presented here is thus a two stage process: initial capillary invasion followed by deformation and fracturing, where the required pressure is always set by the pore entry pressure of the close-packed material of the compaction front, regardless of the frictional properties of the medium.

\subsubsection{Effect of Granular Friction (grain shape)}

The effect of grain shape was studied by comparing spherical beads with irregular, rough grains (as seen in Figure 5.1), both sieved to a narrower distribution of 75-100 $\mu \mathrm{m}$. The same PIV analysis as performed for Figure 5.6 was used to reveal the differences in the compaction fronts between the two grain shapes, as shown in Figure 5.9 . 
It can be seen in Figure 5.9 (a) that the peak compaction value for the irregular grains is much higher than that for the spherical beads, $1.3 \pm 0.1 \%$ and $0.4 \pm 0.1 \%$ respectively. This higher compaction is explained by the packing of irregular grains having a greater porosity than the packing of spherical beads (0.63 compared to 0.43 ), and with more degrees of freedom, there is a greater potential for reconfiguration and compaction. The measured permeability of the irregular grains $\left(295 \pm 1 \times 10^{-9} \mathrm{~cm}^{2}\right)$ was also larger than for the same sized $75-100 \mu \mathrm{m}$ beads $\left(72 \pm 1 \times 10^{-9} \mathrm{~cm}^{2}\right)$, suggesting the irregular grains do have large pore spaces on average for their size.

Figure 5.9 (b) shows that the compaction front is narrower for the irregular grains: $\approx 4.5 \mathrm{~mm}$ compared to $\approx 6 \mathrm{~mm}$ for spherical beads. This is because the irregular grains will have a higher effective friction against the cell walls than the smooth beads, meaning a narrower front is needed to hold back the same pressure. As an indirect measure of frictional properties, the angle of repose $\alpha$ for dry irregular grains was measured to be $45 \pm 1^{\circ}$, compared to $32 \pm 1^{\circ}$ for spherical beads.

As a consequence of having narrower compaction fronts, it should be expected that fractures in irregular grains will be able to approach closer to each other than those in spherical beads, increasing the fracture density $D$ in the system. This result is observed in the fracture patterns shown in Figure 5.10, where the irregular grains in (b) are seen to form a denser pattern than the spherical beads in (a). Measurements of fracture density (fracture length divided by convex hull) on three replicate experiments on each grain type (Figure 5.10 (e)) show that irregular grains indeed display a higher average fracture density of $1.4 \pm 0.1 \mathrm{~cm}^{-1}$ compared to $1.0 \pm 0.1 \mathrm{~cm}^{-1}$ for the spherical beads.

The measured gas pressure at the onset of fracturing (red triangles in Figure 5.10(e)) is considerably lower for the irregular grains $(3500 \pm 100 \mathrm{kPa}$ compared to $8500 \pm 100 \mathrm{kPa}$ for spherical beads). This observation supports the explanation presented in the previous section that the fracturing pressure is set by the capillary entry pressure as opposed to the frictional resistance of the material. The irregular shape of the grains creates larger pores, requiring lower gas pressure for capillary invasion as the precursor to fracturing. The effect of the higher frictional resistance is that thinner compaction fronts are required to balance a given capillary pressure. The higher fracture density for irregular grains is thus a result of two separate effects: Larger pores means lower capillary pressure acting to expand the fracture, and higher friction means narrower compaction fronts are required to halt the expansion.

Another noticeable difference is that the irregular grains have wider fractures (mean fracture width of $0.72 \pm 0.10 \mathrm{~mm}$ compared to $0.43 \pm 0.10 \mathrm{~mm}$ for beads, see Figure 5.10 (c) and (d)). Recall that the width of the gas filled fracture can be estimated as $W=2 \Xi L$. Figure 5.9 shows that the compaction $\Xi$ is over three times larger for the irregular grains, explaining the larger $W$ despite the irregular grains having thinner compaction fronts $L$. 
(a)
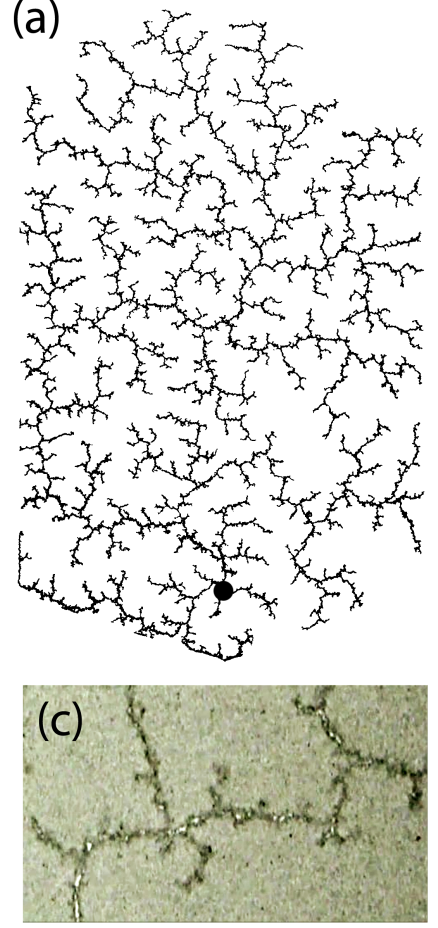

(b)

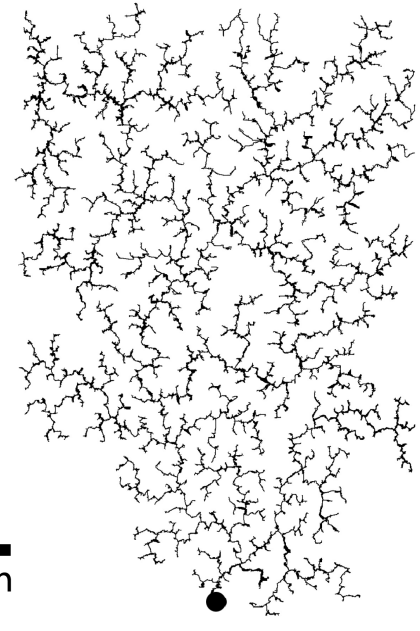

$\overline{50 \mathrm{~mm}}$

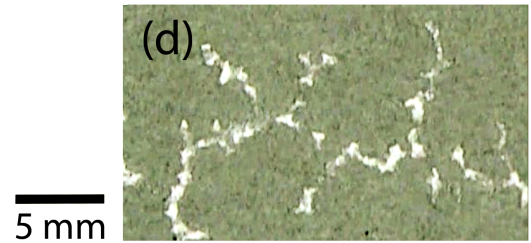

(e)

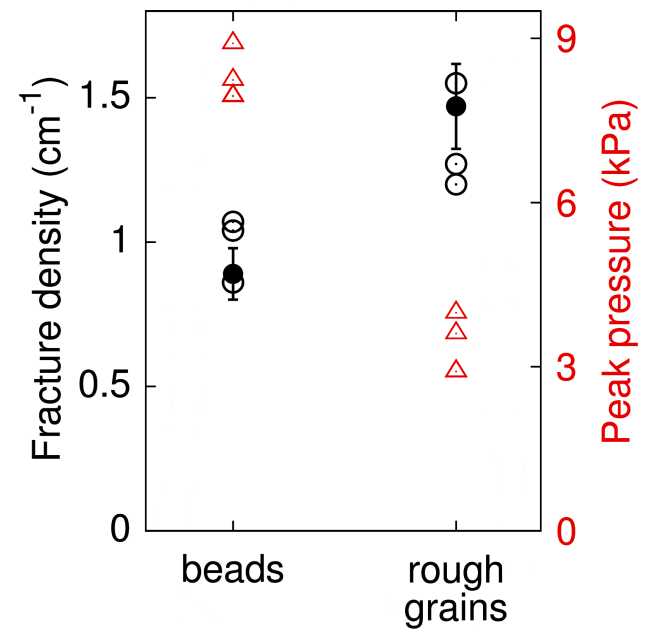

Figure 5.10: Effect of grain shape. Full fracture patterns obtained for (a) spherical glass beads and (b) irregular grains sieved to the same $75-100 \mu \mathrm{m}$ size range Images (c) and (d) illustrate the difference in fracture width and appearance between the beads and irregular grains. The narrower fractures in the beads cause the back-illumination to scatter giving a dark appearance while the wider fractures for irregular grains allow the illumination straight through giving them a white appearance. (e) Measured fracture density (open circles), peak pressure (red triangles), and model results for fracture density predicted by Equation 5.7(filled black circles), with each symbol representing a single experiment like those displayed in (a) and (b).

\subsubsection{Analytical model for fracture density}

The expected width of a compaction front may be estimated from the frictional properties of the granular material together with a simple model for stress transmission within the 
packing. Stress imposed on a granular medium propagates and disperses via grain-grain contacts. The capillary forces at the fracture boundary exert a stress on the interfacial grains with a mean component in the horizontal, in-plane direction. A significant portion of this stress gets redirected towards the confining plates, thus increasing the friction force [22]. We assume a Janssen relation that states that the out-of-plane stress $\sigma_{z z}$ is proportional to the applied in-plane stress $\sigma_{x x}$ by $\sigma_{z z}=\kappa \sigma_{x x}$, where $\kappa$ is the Janssen coefficient, found in simulation to be $0.58 \pm 0.05$ for spherical beads [48, 49]. The fracturing pressure $P_{F}$ that needs to be applied at the fracture interface in order to displace the accumulation front increases exponentially with $L$ according to the following relation which was derived in Chapter 2.1.6 [22, 49]:

$$
P_{F}=P_{T} \exp \left(2 \mu \kappa \frac{L}{b}\right)
$$

where $\mu$ is the static coefficient of friction (estimated, using the angle of repose as $\mu \approx$ $\tan \alpha), b$ is the plate spacing and $P_{T}$ the threshold pressure at the far side of the front, i.e. the minimum pressure needed to compact the granular bed; this was taken as equal to the gravitational stress half-way between the plates, taking into account buoyancy and porosity, i.e.

$$
P_{T}=\frac{1}{2} b \rho_{e}\left(1-n_{0}\right) g
$$

where $\rho_{e}$ is the effective density of glass in water, $n_{0}$ is uncompacted porosity and $g$ acceleration due to gravity.

Assuming that the compaction fronts are much wider than the internal width of the gas-filled fracture, spatial fracture density may be estimated simply as

$$
D=\frac{1}{W+2 L} \approx \frac{1}{2 L}
$$

And as $L$ may be estimated using Equation 5.4 and $P_{F}$ may itself be estimated from grain size as in Equation 5.3, it follows that the spatial density of the fracture pattern may be estimated directly from the basic material properties $\mu, n, \theta$ and $d$ without reference to PIV results or pressure measurements. This final equation is as follows:

$$
D=\frac{\mu \kappa}{b}\left(\ln \left[\frac{8 a \gamma \cos \theta}{b \rho_{e} d(1-n) g}\right]\right)^{-1}
$$

where $a$ is the prefactor from Equation 5.3, found to equal 2.0 for typical fracturing pressure. This value found for $a$ only applies to the spherical beads, and a measured $P_{F}$ of $3.1 \mathrm{kPa}$ was used for the irregular grains in place of Equation 5.3 . 
The parameters relating to the frictional resistance of the compaction front; $\mu, \kappa$ and $b$ are outside the logarithm in Equation 5.7 and are the parameters to which $D$ is most sensitive. Figure 5.10 (e) compares predicted (Eq.(7)) and measured fracture densities. The model correctly predicts an increasing fracture density for the rough grains which is a result of higher friction and lower fracturing pressure producing narrower compaction fronts, allowing the fracture branches to approach each other closer. The model provides a close approximation to measured values, predicting fracture densities of 0.89 and $1.47 \mathrm{~cm}^{-1}( \pm 10 \%)$ for the $75-100 \mu \mathrm{m}$ beads and the irregular grains respectively, in agreement with measured values of 1.0 and $1.4 \mathrm{~cm}^{-1}$. The close match is surprising given the simplicity of the model.

The parameters originating from the capillary stress exerted by the gas fracture on the granular packing $(\gamma, \theta, d)$ are less influential on the emerging fracture density $D$ (being inside the logarithm). The model therefore explains the observed insensitivity of $D$ as a function of grain size. The black line in Figure 5.7 (b) shows model results compared to measured $D$ values (open circles), again reproducing the observed trend with a good match in values. Equation 5.7 predicts only a $10 \%$ increase in fracture density with grain size (including bead size dependence of porosity) over the range of sizes studied.

The capillary stress at the fracture interface is assumed proportional to $\gamma \cos \theta$ and is maximal for high gas-liquid surface tension and for perfectly wetting $(\theta=0)$ materials. A higher capillary stress at the interface allows the fractures to expand wider, growing larger compaction fronts separating the fractures, ultimately resulting in a lower fracture density $D$. Our experiments use highly wetting, hydrophilic grains $\left(\theta=12^{\circ}, \cos \theta=0.98\right)$. Previous studies have found wettability to significantly influence multiphase invasion patterns in fixed porous media models [73-75]. We have not investigated the effect of wetting on the fracturing process, however, the assumptions of the model imply that fracturing is driven by the capillary forces exerted at the interface, which tend to zero for non-wetting conditions $\left(\theta=90^{\circ}\right)$.

\subsubsection{Dependence on Gas Injection Rate}

The rate of gas injection $Q$ into 53-100 $\mu \mathrm{m}$ beads was varied across five orders of magnitude, from 0.001 to $100 \mathrm{ml} / \mathrm{min}$. The collected results are presented in Figure 5.11 . Figure 5.12 shows quantitative aspects of the same results.

From both images and graphs, it is clear that there is no significant variation with rate up to about $0.1 \mathrm{ml} . \mathrm{min}^{-1}$. This is the stick-slip regime described in the previous sections (outside this rate study all experiments were performed at $0.03 \mathrm{ml} / \mathrm{min}$ unless specified otherwise). The rate independence here is to be expected: as a threshold-limited process, the only influence of the rate is the length of time it takes for the pressure to build up to the next threshold, which likely has little influence on the pattern (see Figure 5.3). 

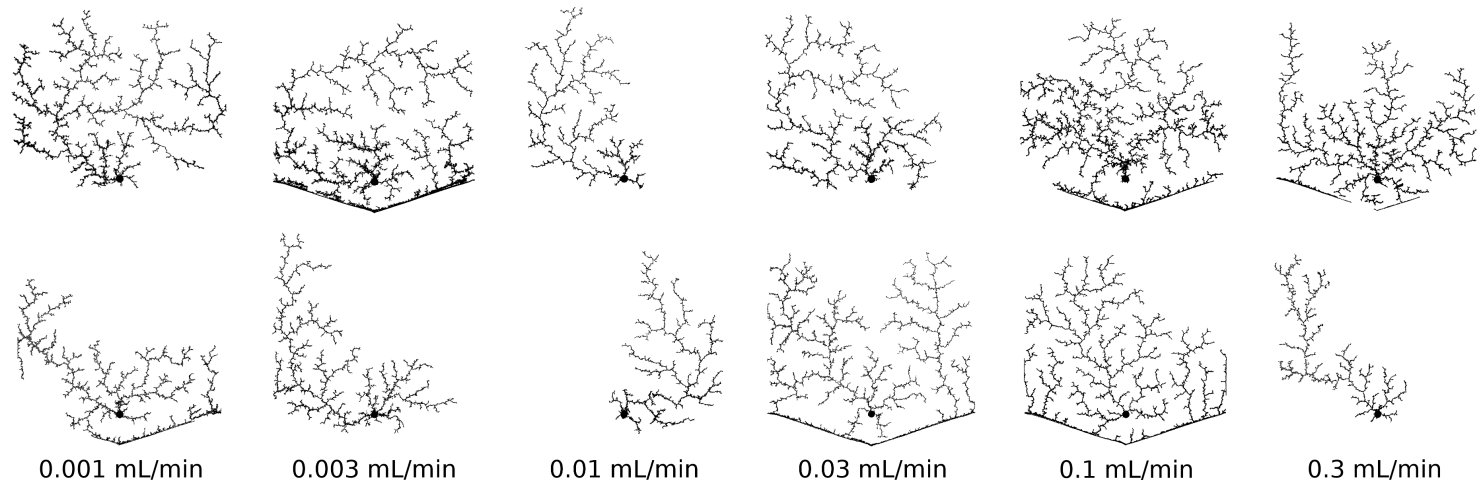

$10 \mathrm{~cm}$
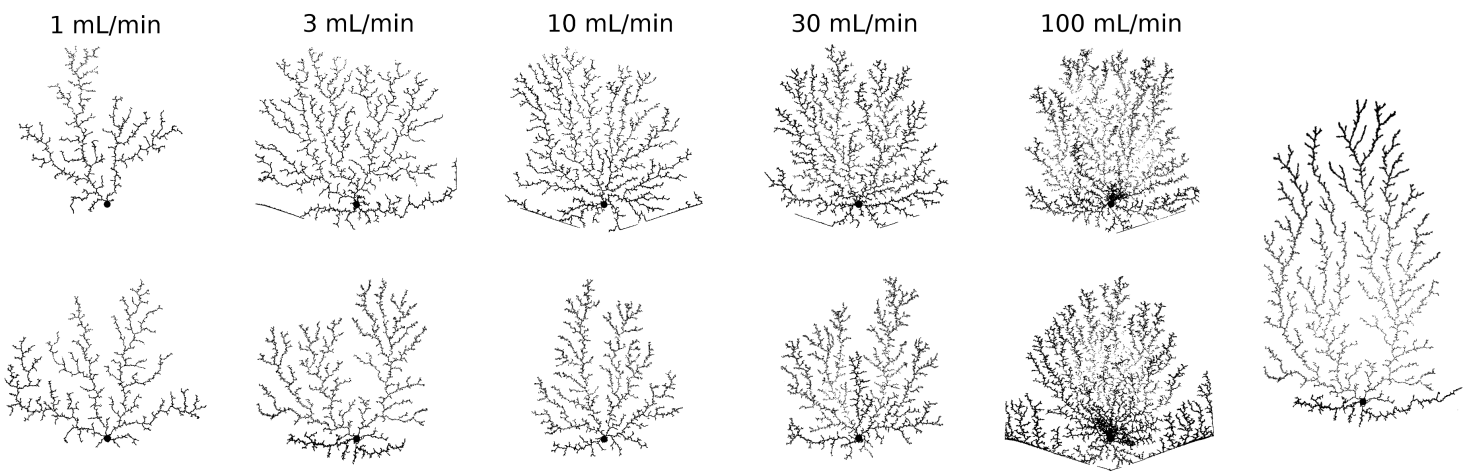

Figure 5.11: Invasion patterns obtained for varying gas injection rates. The image in the bottom right shows a full invasion pattern for $3 \mathrm{ml} / \mathrm{min}$.

From about $0.3 \mathrm{ml} / \mathrm{min}$ onwards, the pattern becomes strongly rate-dependent. Below this rate, the pressure during fracturing rises and falls in the stick-slip manner seen in Figure 5.3, but stays close to a constant fracturing pressure $P_{F}$ during the whole experiment. Above this rate, after the first fractures appear close to $P_{F}$, the pressure continues to build, reaching a maximum and then falling off as the fractures approach the open end of the cell, with no discernible stick-slip fluctuations. This maximum pressure increases with increasing rate, as seen in Figure 5.12. There is thus a critical rate above which the growth dynamics becomes continuous rather than stick-slip.

As well as becoming continuous in time, the movement of the granular bed begins to extend much further from the fractures. At $0.1 \mathrm{ml} / \mathrm{min}$ and below, fractures open by means of local compaction as shown in Figures 5.5 and 5.6. Above this rate, they displace the grains globally rather than merely locally, as illustrated in Figure 5.13. Thus the transition to rate-dependent behaviour reflects a fundamental transition from fracturing of a solidlike, deformable packing, to gas invasion into a granular suspension.

The change in flow behaviour can be explained as a fluidisation transition: above a threshold flow rate $Q_{f}$ the drag force will be sufficient to mobilise the grains and set the whole bed in motion. This concept has already been used to explain pattern transitions in part-filled bead beds [21] and tube geometries [200], and here we use the same approach; the bed will fluidise when the fluid pressure gradient $\nabla P$ is sufficiently large to overcome 


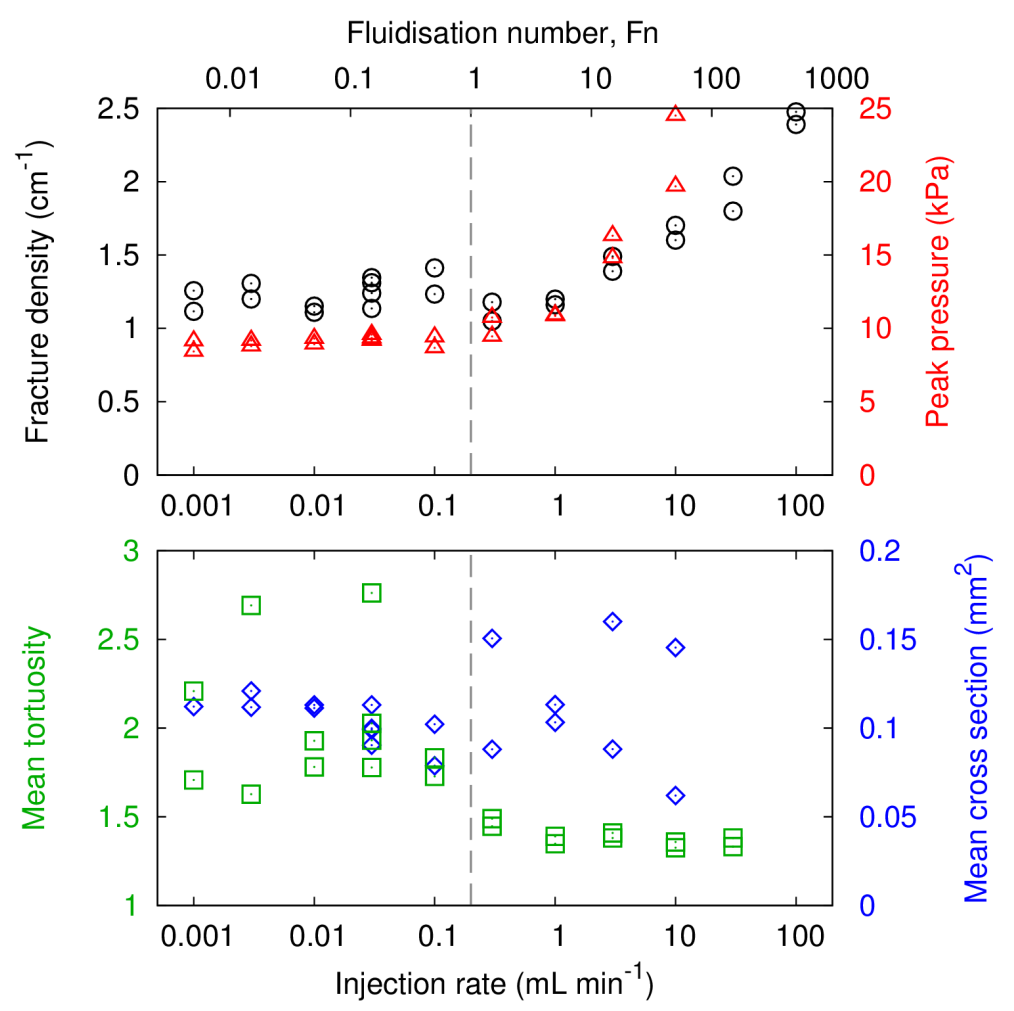

Figure 5.12: Dependence of fracture pattern properties on injection rate. (black circles) fracture density; (red triangles) peak pressure; (green squares) mean tortuosity from inlet; (blue diamonds) mean fracture cross sectional area. Each symbol represents a single experiment. The dashed line indicates the estimated value of $F n=1$, the fluidisation transition.

gravitational friction:

$$
\nabla P>\mu \rho_{e} g
$$

The fluid pressure gradient through the packing according to Darcy's Law [201] is

$$
\nabla P=\frac{\eta Q_{f}}{k A}
$$

where $\eta$ is the viscosity, $A$ the cell cross-sectional area and $k$ the permeability, predicted for monodisperse spherical beads of diameter $d$ by the Kozeny-Carman relation [187]

$$
k=\frac{d^{2}}{180} \frac{n^{3}}{(1-n)^{2}}
$$

where $n$ is the porosity. From Equations 5.8 and 5.9 we can predict the critical fluidisation flow rate $Q_{f}$ as

$$
Q_{f}=\frac{\mu \rho_{e} g k A}{\eta}
$$

In order to make the conclusion more general, we define a new dimensionless number $F n$, the Fluidisation number, which is independent of cell dimensions. $F n$ is the ratio of 

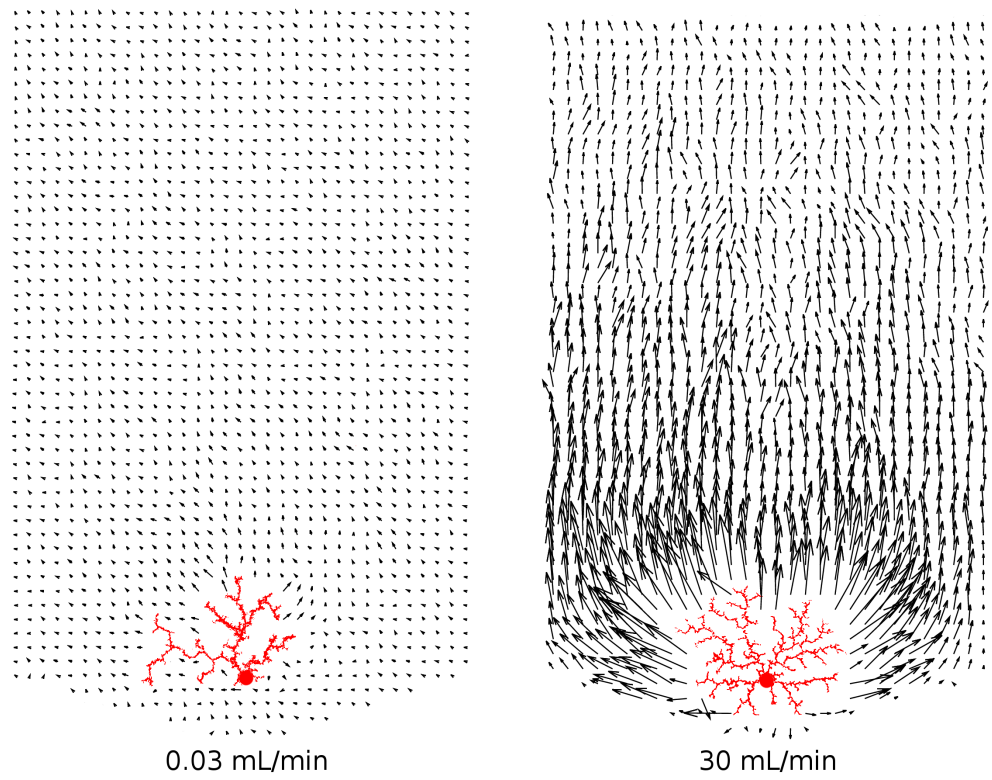

Figure 5.13: Fluidisation transition. Whole-cell PIV showing net displacement of the granular bed exaggerated $50 \times$ at an early stage of growth for (a) 0.03 and (b) $30 \mathrm{ml} / \mathrm{min}$. At low rate (a) there is no discernible grain movement except very close to the fractures; at high rate (b) the whole bed is fluidised and pushed through the cell The width of the PIV fields correspond to the cell width, i.e. $200 \mathrm{~mm}$.

viscous and frictional forces acting on the granular packing:

$$
F=\frac{q \eta}{\mu \rho_{e} g k}
$$

where $q=Q / A$ is the time- and space-averaged Darcy flow rate. $F n$ is equivalent to $Q / Q_{f}$, so for $F n>1$ (high injection rate) we expect the bed to be fluidised, and for $F n<1$ we expect to see stick-slip behaviour with no global movement of the grains. $F n=1$ marks the transition from a frictional regime at low injection rate (low capillary number) to a regime dominated by viscous forces at high flow rate (high capillary number).

Permeability was measured directly $\left(7.2 \pm 0.1 \times 10^{-8} \mathrm{~cm}^{2}\right.$ for spherical and $2.95 \pm$ $0.01 \times 10^{-7} \mathrm{~cm}^{2}$ for irregular), as well as being calculated from equation $5.10(8 \pm 0.5 \times$ $10^{-8} \mathrm{~cm}^{2}$ for spherical and $3 \pm 0.5 \times 10^{-7} \mathrm{~cm}^{2}$ for irregular with a sphericity of 0.5 ), and were found to be in good agreement. Using these values with an estimated $\mu=\tan 32^{\circ}$ from the tangent of the measured angle of repose, we predict $F n=1$ to occur at $0.2 \mathrm{ml} / \mathrm{min}$ for 53-100 $\mu \mathrm{m}$ beads. This value, marked on the graphs in Figure 5.12, is consistent with the flow rate at which the fluidisation transition is observed experimentally. Using the classical definition of the Capillary number, $\mathrm{Ca}=\eta v / \gamma$, with seepage velocity $v=$ $Q /(n A)$, we find the fluidisation transition at $\mathrm{Ca}=10^{-6}$. 
With a fluidised bed, grain displacement occurs globally rather than locally which explains the increasing directionality of fractures at high injection rates. At very high rates the fractures align with the streamlines of flow through the system (compare to Figure 5.13). In Figure 5.12, the increased directionality is expressed as a decreased tortuosity.

The fracture density increases at high rates; with viscous flow pushing the grains through the whole cell, the spacing between fractures is no longer limited by the compression of the intermediate granular bed as discussed in the previous section. The mean cross-sectional area of the gas-filled fractures in contrast shows no clear trend with rate.

The images shown in Figure 5.11 are taken at the moment the fractures first reach $15 \mathrm{~cm}$ from the inlet. Within this first half of the cell the invading air channels at high rates still appear visually fracture-like. However in the second half of the cell the channels widen and take on the appearance of viscous fingers (bottom-right of Figure 5.11), similar to the morphologies observed in granular suspensions with lower granular filling fraction [9, 71].

It should be noted that this experiment was performed with an open boundary condition at one end of the cell, allowing movement of both grains and fluid. If instead the boundary permitted passage of fluid but not grains, then the stick-slip behaviour at $F n<1$ should remain unaffected but the behaviour at $F n>1$ might be markedly different in ways which are beyond the scope of this study [196].

One final rate dependence was seen only at the very highest rate, $100 \mathrm{ml} / \mathrm{min}$. Whereas at lower rates the patterns, once formed, appear fixed and invariant, at this rate the fingers are sufficiently fluidised that they may deform or pinch closed after growth.

\subsection{Conclusion}

We have conducted an extensive series of experiments where saturated, cohesionless beds of granular material were fractured by forced injection of air. Gas-driven fracturing of granular suspensions and saturated packings occur in geological processes such as soil drying, sedimentary methane venting and magma degassing [69, 185, 202], and our results contribute to a deeper understanding of how the multiphase gas/liquid/grain interactions govern fracturing dynamics and emergent fracture network properties. We have found two distinct regimes in our experiments: a frictional regime at low gas injection rate/pressure, and a viscous regime at high injection rate/pressure.

In the frictional regime, the dynamics are dominated by stick-slip growth of fracture branches. A period of inactivity is followed by sudden fracturing, followed by another period of inactivity and so on. The pressure builds and drops in a saw-tooth fashion familiar from other intermittent systems such as frictional sliding [188] and earthquakes [189]. We attribute the stick-slip intermittency in the growth of individual fractures to the nonlinear frictional response of the granular material. 
PIV analysis of grain displacement reveals a compaction front surrounding the fractures (Figure 5.6). Our results show that it is ultimately the frictional resistance of this jammed compaction front that halts the lateral widening of the fractures, balancing the capillary stress exerted by the gas on the fracture interface. In a quasi-2D system like our Hele-Shaw cell, the friction is absorbed by the confining plates. It is at this point not clear whether a similar compaction process would take place in an unconfined 3D geometry, however we speculate that local compaction of material surrounding internally weakened conduits could increase the structural stability of gas migration pathways in sediments and crystal-rich magma.

The intermittent dynamics and the growth of the compaction front have implications for the fracturing pressure. The maximum pressure that the front can withstand is set by the capillary entry pressure of the pores of the compacted material. Below this pressure, the system responds to an increasing gas pressure by mobilizing friction (e.g. thickening the front). This means that nucleation of a new fracture length will not take place until the gas starts to penetrate the front by capillary invasion of the pores, triggering accelerated growth by locally fluidising the material. The pressure at the onset of fracturing is therefore determined by the capillary properties (pore size, wetting) of the material, and is independent of the frictional or deformational properties of the medium.

In engineering applications of pneumatic fracturing (e.g. soil remediation [14, 15], stimulation of hydrocarbon reservoirs [186, 203]), the objective is to increase the rate of fluid exchange with the bulk by generating a network of conductive paths. The rate of fluid exchange will to a large degree be determined by how closely spaced the fracture branches are, i.e. the spatial density of the network. The fractures in our experiments grow by tip-splitting to form a rooted binary tree network. The self-avoiding nature of the network, and the minimum separation distance between neighbouring branches is set by the compaction fronts. Our experiments display a characteristic length scale: a fairly uniform separation distance between fracture branches, which we attribute to confinement effects (the cell has only one open edge), and "cooperative" fracturing in a local process zone. The resulting spatial fracture density is to a first approximation determined by the width of the gas-filled fracture and the compaction fronts either side $\left(D=(W+2 L)^{-1}\right)$, where the thickness of the compaction front $L$ depends on both granular friction and capillary pressure. Our experiments with irregular grains produced higher fracture density compared to smooth beads because: (1) higher friction gave narrower compaction fronts, and (2) larger pores gave lower fracturing pressure. 
The change in dynamics from stick-slip frictional to continuous (viscous) invasion occurs as a result of a fluidisation transition [21, 200]: when the flow velocity of displaced fluid through the granular bed is high enough that viscous drag overcomes the friction between the grains and the cell, the entire granular bed is set in motion. The fractures grow into a partially fluidised bed, where granular material is pushed towards the open boundary. The fracturing process becomes rate-dependent, and we observe an increasing fracture density as a function of rate. We note the importance of the open boundary for this particular observation, and that work by other authors on granular displacement with closed boundaries for grain flow produce a different set of compaction dynamics [78, 196].

Our results show that multiphase flow and fracturing of granular materials is governed by a complex interplay between capillary, frictional and viscous forces, and we demonstrate the effect of material properties and local deformation on the emergent patterning of the evolving fracture network. While open questions remain regarding the fundamental forces at play, a big challenge for future work will be to incorporate more realistic subsurface conditions such as bulk 3D behaviour and heterogeneity to successfully model sedimentary venting and magma degassing dynamics, and to optimise pneumatic fracturing processes to achieve maximum fluid exchange rates, thereby reducing operation time and costs. 


\section{Chapter 6}

\section{Rheology of Shear Thickening Suspensions}

\subsection{Introduction}

As discussed earlier, the rheological properties of a fluid determine what happens when it is disturbed. Viscosity describes the rate at which energy is dissipated in a fluid under shear, defined as the ratio between the shear stress and shear rate in steady flow. For a Newtonian fluid, viscosity is constant property of the material. However, a complex nonNewtonian fluid has a complex response, for which a function governs its viscosity rather than a constant.

This chapter is made up of preliminary work which supports the following chapter on shear thickening and DST multiphase invasion patterns. Here the rheology of cornstarch suspensions of varying volume fractions of cornstarch (in accordance to the volume fractions use in the following chapter) are studied in a rheometer. The Wyart-Cates theoretical model that describes DST behaviour is then presented in the context of these measurements, comparing the theoretical flow-curves with the measure flow-curves. This favourable comparison prepares the use of the model in explaining the novel Hele-Shaw cell experiments performed in the following chapter.

The experimental setup in this chapter was designed and performed by Miles Morgan with the supervision of myself. The results were analysed by myself with discussions with Miles Morgan and the supervision of Bjørnar Sandnes. 


\subsection{Rheology Methods}

\subsubsection{Cornstarch Suspensions}

The cornstarch was prepared in suspensions with varying concentration, between $\phi=$ $0.23-0.56$, by mixing a weight ratio of cornstarch and deionised water. However, the weight and size of cornstarch particles varies with how much moisture it has absorbed. This variation is sensitive to the atmosphere as the humidity changes by day as well as of course when it is submerged in water.
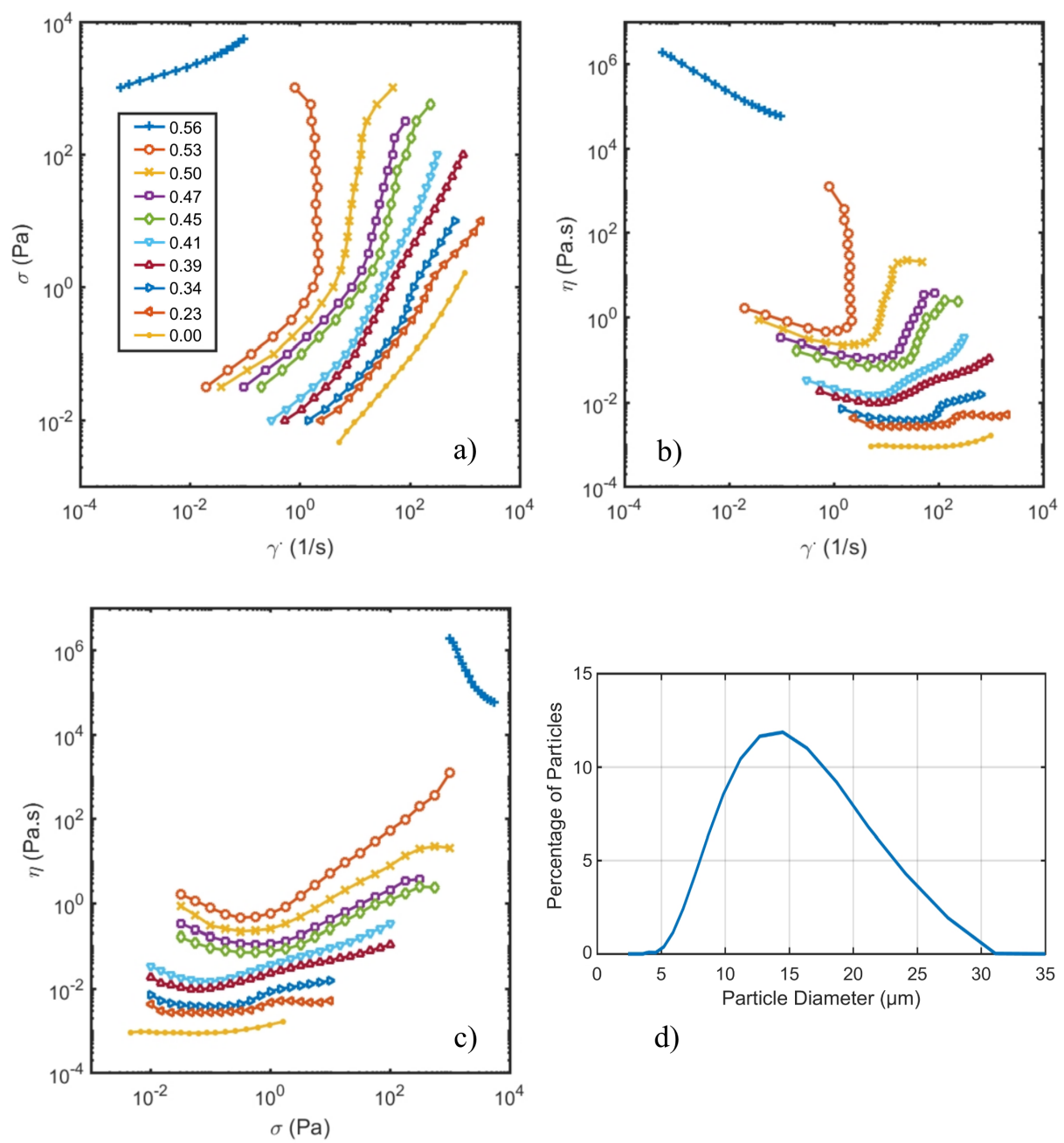

d)

Figure 6.1: Rheology of cornstarch suspensions. a) Measured shear rate at imposed shear stress of the suspensions. b) The calculated viscosity of the suspensions plotted against shear rate showing DST for $\phi=0.45$ or higher volume fractions. c) The calculated viscosity of the suspensions plotted against shear stress showing that DST occurs at approximately the same stress for the $\phi=0.45$ or higher volume fractions. d) Size distribution of cornstarch particles in water suspension showing an average particle diameter of $16.1 \mu \mathrm{m}$. 
A consequence of this is that it is difficult to know the volume fraction of weighed "dry" cornstarch particle once in suspension. To know the volume fraction, the amount of water initially absorbed from humidity as well how much cornstarch particles swell with absorption needs to be understood. In literature this issue was not initially tackled, instead many papers quoted weight fractions which are proportional to $\phi$ with an unknown constant [110, 114, 115, 125]. For example, Hermes et al. (2016) [125] state "The particles swell in our solvent, so that we cannot access the volume fraction. We therefore quote mass fractions." A method that has been used, instead of quoting weight fractions, is to assume the grains are dry and calculate the volume fraction using the density of cornstarch $1.6 \pm 0.05 \times 10^{3} \mathrm{~kg} \cdot \mathrm{m}^{-3}[114,122,151]$. However, this does not account for swelling or the atmospheric moisture in the cornstarch. As such, there has been some difficulty in cornstarch rheology literature when comparing results between different laboratories.

Jaeger's group [120, 123] have recently addressed this problem by accounting for the fraction of cornstarch volume increase (swelling) caused by absorption when submerged in water, which is estimated to be $\lambda \approx 0.3$ [204, 205], such that $\phi=(1+\lambda) \phi_{v}$, where $\phi_{v}$ is the volume fraction when dry (calculated using weights and densities). In addition, they included a $\beta$ fraction that factored in the initial moisture content absorbed by particles caused by humidity in their labs.

$$
\phi=\frac{(1-\beta) m_{c s} / \rho_{c s}}{(1-\beta) m_{c s} / \rho_{c s}+\beta m_{w} / \rho_{w}}(1+\lambda),
$$

Where $\phi$ is the volume fraction of cornstarch in the final sample, $m_{c s}$ is the measured mass of cornstarch, $\rho_{c s}$ is the density of cornstarch $\left(1.6 \pm 0.05 \times 10^{3} \mathrm{~kg}^{\mathrm{m}} \mathrm{m}^{-3}[114,122\right.$, 151]), $m_{w}$ and $\rho_{w}$ is the mass and density of deionised water respectively, $\lambda$ is the pore space in cornstarch that can absorb water when fully submerged $(\lambda \approx 0.3[123])$, and $\beta$ is the pore space in cornstarch that has absorbed water in natural room humidity $(\beta \approx$ 0.13 [121, 204]). The variance that results from differing humidities between days that experiments were conducted on is minimal, a change of approximately $\pm 1 \%$ of the sample mass [120, 123]. However, to reduce the impact of this daily variation, before weighing and mixing the cornstarch it was first prepared in a vacuum chamber for 45 minutes to remove ambient moisture from the sample. This process was found to remove $\approx 20 \pm 5 \%$ giving $\beta=0.10 \pm 0.01$ which was used for all calculations.

Care was taken during mixing to avoid introducing bubbles of air to the suspensions, however it has been found that small air pockets do not significantly affect the rheological properties of cornstarch suspensions [120]. The prepared sample was stored in a sealed container to avoid evaporation before it was used. The experiment was run within minutes of the mixture being poured into the cell to avoid settling, which takes place over several hours even without a density matched liquid [114]. 


\subsubsection{Experimental Challenges of Rheology}

The simplest example of rheometry is drag flow between two infinite parallel planes. Infinite rotating cylinders, cones, or disks can create an equivalent flow. In these rotational flows, a surface remains stationary while another rotates, generating a shear rate and shear stress in the fluid that rests between them. Obviously, an infinite plane is unachievable so all rheological experiments require considerations with regards to geometric approximations, boundary effects, particle size, and fluid behaviour. Recall from Chapter 2.2.3 that when this relationship is plotted on an $\sigma-\dot{\gamma} \log$-log scale a Newtonian fluid will have a flow curve with a gradient of 1 , a shear-thinning fluid will have a gradient less than 1 , and a shear-thickening fluid will have a gradient more than 1 .

For a rotating surface the velocity at the centre is zero while the velocity at the edge is maximal. This is an issue if the surface is flat and the gap height is constant as the shear rate will vary across a sample being tested. A conical plate is an "ideal" geometry for rheological measurements because the gap height also varies from approximately zero in the centre to maximal at the edge to maintain a constant shear rate across the whole sample that is only dependent upon the angular velocity $\Omega$ and the angle of the cone $\alpha$ :

$$
\dot{\gamma}=\frac{\Omega}{\alpha}
$$

The shear stress is also constant across the cone and is given by:

$$
\sigma=\frac{3 T}{2 \pi R^{3}}
$$

where $\mathrm{T}$ is the torque, and $\mathrm{R}$ is the radius of the cone.

However, there are issues with cone geometries. The angle of the cone must be shallow, the gap height cannot be varied, and the centre must be close to the bottom plate $(\sim 50 \mu \mathrm{m})$; this limits the shear rates than can be reached [206]. The narrow height for the cone is a critical issue with suspensions, as a gap height of 10 times the size of the particles is recommended so that the fluid can be considered continuum where confinement effects are negligible [207, 208]. The cornstarch particles, when suspended in water, were measured to have a diameter from between 5 to $30 \mu \mathrm{m}$ with an average diameter of $16.1 \pm 0.1 \mu \mathrm{m}$ using a Mastersizer 3000E, see Figure 6.1. Hence a gap spacing of at least $300 \mu \mathrm{m}$ would be appropriate for cornstarch particles, which makes a cone geometry not applicable.

Parallel plate geometries are not constrained by a narrow height and so offer a solution to this problem, though with a caveat. As mentioned the shear rate is not constant across a flat plate but as we are measuring the point at which the sample begins to experience a shear thickening response, the highest shear rate is precisely what we are interested 
in. Therefore, the shear rate at the edge of the plate is used:

$$
\dot{\gamma}=\frac{R \Omega}{h} .
$$

In addition, smaller plates can be used to minimise the distance between different shear rates experienced across the sample.

A premise of accurate rheometry is that the sample fluid "sticks" to the constraining surfaces while in motion, this is called the no-slip condition. However, slipping can occur, often caused by rapid shear rates, decreasing the measured stress and therefore the measured viscosity. This would appear as shear-thinning as the effect would exaggerate with increasing shear rates [207]. When this occurs, roughened plates are utilised to prevent slip, however this can introduce uncertainties into the true height of the gap spacing [207]. The fluid itself can undergo undesirable effects that introduce further complications to rheometry. Secondary flows within the sample fluid, also often caused by rapid shear rates, can increase the torque in the system. This would be recorded as an increase in viscosity and therefore as shear-thickening as the effect will grow with increasing shear rate. Less viscous fluids are more prone to this undesirable effect and it can be avoided by decreasing the gap size [207].

\subsubsection{Experimental Method}

The cornstarch suspensions were tested in Bohlin Gemini HR Nano Rheometer, using a smooth stainless steel parallel plate geometry. Parallel plates were used over conical because of spacing required for particle size and smooth plates were used over roughened because in measurements slippage was only observed at shear rates after thickening had occurred, which was beyond the area of interest and therefore not consequential. As a cornstarch suspension's rheological behaviour is heavily stress dependant we imposed a step increasing shear stress on the suspensions across five orders of magnitude, from $10^{-2}-10^{3} \mathrm{~Pa}$. A delay time of $5 \mathrm{~s}$ was set for the rheometer to adjust between measurements and a measurement window of $5 \mathrm{~s}$ was used. The shear rate at which the step stress was obtained for each volume fraction was measured to calculate their viscosities. 
Considering the effects outlined above, two different rheometry geometries were utilised: one for the five most dilute suspensions $\phi=0.00,0.23,0.34,0.39$, and $0.41 \pm 0.01$, and the other for the five most dense suspensions $\phi=0.45,0.47,0.50,0.53$, and $0.56 \pm 0.01$. The denser suspensions were tested with a gap height of $0.8 \mathrm{~mm}$ and plate diameter of $20 \mathrm{~mm}$. The gap height was more than double the recommended minimum for the particle size as the dense suspensions were found to be more susceptible to confinement effects. The turbulence associated with larger gaps was less of a concern for the denser suspensions because of the higher viscosities involved. A relatively small plate diameter was also used to minimise the distance between different shear rates and stresses experienced across the sample since the DST response is so sensitive to shear stress.

The dilute suspensions were tested with a smaller gap height of $0.4 \mathrm{~mm}$ and a wider plate diameter of $40 \mathrm{~mm}$. The height used was deemed to be the narrowest gap size appropriate for particles of this size to reduce turbulence, which was the largest consideration for the less viscous samples. Turbulence was observed at high shear rates but in experimental regions beyond interest. A wider plate was used with the less dense suspensions to reduce the angular velocity required of the rheometer to reach the desired shear rates at the lower height to minimise secondary flows associated with less viscous samples. As a solid-like response was not expected or recorded in the least dense suspensions, the larger distance between different shear rates experienced across the sample was of less concern. 


\subsection{Rheology Measurements}

Figure 6.1 presents the same flow curves in three different plots. Note that throughout this Chapter and the following Chapter flow curves will be plotted in the conventional way, with shear rate on the x-axis, even though a stress-controlled experiment is used and the shear rate is the independent variable. Plot a) shows imposed shear stresses and measured shear rates for the volume fractions used. Plot b) shows the calculated viscosity of the suspensions plotted against the measured shear rate, illustrating some shear thickening in all of the suspensions and instances of discontinuous shear thickening for volume fractions of $\phi=0.45$ and higher. As the volume fraction increases, the overall viscosities increase and the shear rate at which thickening is seen is lower. During the discontinuous shear thickening regime, small increases in the shear rate increase the viscosity of the suspension orders of magnitude until a new plateau is reached. For the second highest volume fraction $\phi=0.53$ the shear rate could not be increased past $2 \mathrm{~s}^{-1}$ with the stresses imposed by the rheometer as the sample jammed, so the next level of viscosity could not be measured. Note that for the RCP jamming volume fraction $\phi_{c}=0.56$ the viscosity begins orders of magnitude above the rest and the equipment jammed at moderate shear rates. Plot c) shows the calculated viscosity of the suspensions plotted against the imposed shear stress, where it can be seen that for the volume fractions at which DST occurs the thickening begins at approximately the same stress, $0.5 \pm 0.1 \mathrm{~Pa}$. The curve for $\phi_{c}$ appears stunted here because of its high yield stress.

Finally, measurements at the highest shear rates for water exhibit apparent slight thickening, which ought not be possible for the purely Newtonian fluid. We suspect this is an erroneous measurement caused by secondary flows, at shear rates that are beyond our area of interest, as discussed earlier. Overall, these measurements are in agreement with flow curves recorded for similar volume fractions in prominent reports on the rheology of cornstarch suspensions [110, 115, 119, 125]. Though it is beyond the focus of this work, it should be noted that there are reports in literature of the onset of shear thickening at lower shear rates with decreasing gap size related to the dilatancy model of shear thickening discussed in Chapter 3.2.3 [115, 209]. This could be an explanation for the slight disparity between the onset stress of thickening $\sigma_{c}$ between the narrower gap geometry $\sigma_{c} \approx 0.1 \mathrm{~Pa}$ and the taller gap geometry $\sigma_{c} \approx 0.5 \mathrm{~Pa}$.

\subsection{Theory}

Following Chapter 2.2.2 a suspension approaches the RCP limit for spheres the viscosity begins to drastically increase until the system jams. Models that replicate this jamming at $\mathrm{RCP}$ with frictionless hard-spheres take the established form of

$$
\eta_{r}=\left(1-\frac{\phi}{\phi_{c}}\right)^{-\beta}
$$


Where $\phi_{c}$ is the RCP volume fraction and $\beta$ is a constant that ranges from 1.5-2 [52-54] taken here to be $2[54,146]$. Note that in a suspension with frictional spheres the system will jam at a lower volume fraction than the RCP [146]. This model does not explain stress induced shear thickening behaviour, only the increase in viscosity as a result of an increase in $\phi$.

\subsubsection{Frictionless to Frictional}

Wyart and Cates (2014) [146] propose a steady flow theory for the rheology of shear thickening non-Brownian suspensions. This model treats the suspension as existing between three states, a frictionless state with a traditional RCP jamming volume fraction of $\phi_{c}$, a fully frictional state with a lower jamming fraction of $\phi_{m}$, and a transitional state with a jamming fraction of $\phi_{J}$ that moves between $\phi_{c}$ and $\phi_{m}$, see Figure 6.2 a).

They consider a repulsive force between the hard frictional particles that interacts at a range far smaller than the particle radius. Its strength determines a critical pressure $P_{c}$ for which the pressure experienced by particles $P$ must exceed to allow frictional contact [112]. As such, the suspension is frictionless when $P \ll P_{c}$ and becomes frictional when $P \gg P_{c}$. Here a dimensionless reduced particle pressure $P_{r}$ can be defined as a ratio of the
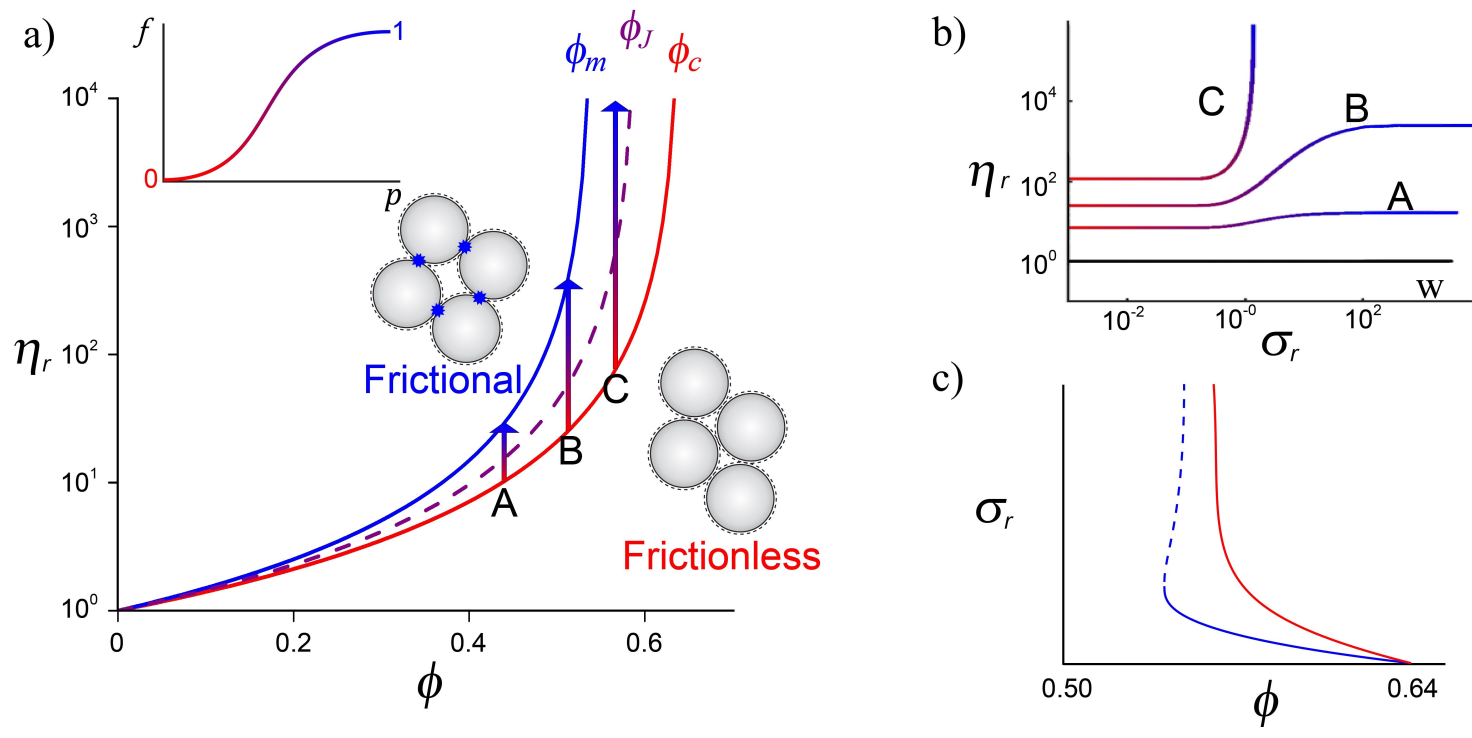

Figure 6.2: Graphs illustrating principles of Wyart-Cates theory. a)

Kreiger-Dougherty function for jamming at a critical volume fraction for a suspension with a system that is frictionless $\phi_{c}$, frictional $\phi_{m}$, and intermediate $\phi_{J}$; inset plots the function $f=\exp \left(\frac{-c}{P_{r}}\right)$ for the fraction of particles in frictional contact that satisfies the condition of $f=0$ for $P_{r} \ll 1$ and $f=1$ for $P_{r} \gg 1$. As the particle pressure is increased the system transitions from frictionless (lower red curve) to frictional (upper blue curve). b) Example flow curves for different concentration suspensions as a function of stress and viscosity, with W showing water, A showing CST, B showing DST, and C jamming. c) Stress condition for DST (blue line) and jamming (red line) for different concentrations. 
two

$$
P_{r}=\frac{P}{P_{c}}
$$

where $P_{r} \ll 1$ when the system is frictionless and $P_{r} \gg 1$ when it is frictional.

The fluid transitions smoothly from a frictionless system to a frictional one as the fraction of particles in contact with another increases from 0 to 1 with increasing particle pressure $P$. A function $f$ is defined to model this behaviour:

$$
f\left(P_{r}\right) \in[0,1] .
$$

Where $f$ is a function that produces a dimensionless number for the fraction of frictional contacts that depends on the particle pressure ratio $P_{r}$. This function can take any form that fits these conditions but Wyart et al. found the following to fit simulations and experiment [146]

$$
f=\exp \left(\frac{-c}{P_{r}}\right)
$$

where $c$ is a scaling factor determining how much greater $P_{r}$ needs to be than 1 for a significant fraction of frictional contacts to be made.

This function can define how the jamming volume fraction of the suspension transitions between the frictionless $\phi_{c}$ and the frictional $\phi_{m}$ as follows:

$$
\phi_{J}\left(P_{r}\right)=\phi_{m} f\left(P_{r}\right)+\phi_{c}\left(1-f\left(P_{r}\right)\right)
$$

The result of this is that a suspension with a volume fraction lower than the traditional jamming fraction of $\phi_{c}$ can transition from a flowing frictionless state to a jammed frictional state with increasing particle pressure.

\subsubsection{Shear Stress}

So far only the pressure the particles experience have been discussed, to be relevant to the suspensions as a whole, the stress on the fluid needs to also be discussed. The pressure experienced by the suspension particles $P$ is assumed to be proportional to the macroscopic shear stress $\sigma$ exerted on the suspension according to a macroscopic coefficient of friction $\mu$ [125, 146]

$$
\sigma=\mu P
$$

Therefore the critical contact pressure $P_{c}$ is also proportional to a critical contact stress $\sigma_{c}$. A reduced shear stress can then be defined using these proportionalities:

$$
\sigma_{r}=\frac{\sigma}{\sigma_{c}}=P_{r}=\frac{P}{P_{c}}
$$


As $\sigma_{r}$ and $P_{r}$ are equivalent, the former can be substituted for the latter in Equation 6.8 and then Equation 6.9 is used to give:

$$
\phi_{J}\left(\sigma_{r}\right)=\phi_{m} f\left(\sigma_{r}\right)+\phi_{c}\left(1-f\left(\sigma_{r}\right)\right)
$$

\subsubsection{Viscosity}

As discussed earlier, it is known that the viscosity of a suspension diverges from the interstitial fluid viscosity as the volume fraction approaches jamming limit $\phi_{c}$, see Figure 6.2 a). However in a shear thickening suspension the jamming volume fraction $\phi_{J}$ is not a constant and is instead a function of the shear stress as outlined in Equation 6.12. Substituting this into Equation 6.5 gives a stress dependant viscosity:

$$
\eta_{r}=\left(1-\frac{\phi}{\phi_{J}\left(\sigma_{r}\right)}\right)^{-2}
$$

\subsubsection{Shear Rate}

Using the standard relation between shear stress, shear rate, and viscosity

$$
\sigma=\dot{\gamma} \eta
$$

both sides can be divided by the critical shear stress to get the reduced stress

$$
\sigma_{r}=\frac{\dot{\gamma} \eta}{\sigma_{c}}
$$

then $\eta$ is substituted with the interstitial and reduced viscosities (see Equation 2.21 in Chapter 2.2.2) to obtain the following:

$$
\sigma_{r}=\frac{\dot{\gamma} \eta_{s}}{\sigma_{c}} \eta_{r}
$$

From here a dimensionless reduced shear rate $\dot{\gamma}_{r}$ is defined, by isolating variables from the reduced stress $\sigma_{r}$ and the reduced viscosity $\eta_{r}$. It is a ratio of the shear rate and a critical shear rate made from the constant critical shear stress and the constant interstitial viscosity:

$$
\dot{\gamma}_{r}=\frac{\dot{\gamma} \eta_{s}}{\sigma_{c}}
$$

substituting this into 6.16 unsurprisingly gives the traditional definition of viscosity in a reduced form:

$$
\sigma_{r}=\dot{\gamma}_{r} \eta_{r}
$$


Finally, Equations 6.12, 6.13, and 6.18 are used and rearranged for the reduced shear rate to obtain:

$$
\dot{\gamma}_{r}=\sigma_{r}\left(1-\frac{\phi}{\phi_{m} f\left(\sigma_{r}\right)+\phi_{c}\left(1-f\left(\sigma_{r}\right)\right)}\right)^{2},
$$

which is a relationship between the reduced shear rate, reduced shear stress, and reduced viscosity, where the reduced viscosity is a function of the volume fraction and reduced shear stress.

\subsection{Results and Discussion}

\subsubsection{Theoretical Curves}

Equation 6.19 produces flow curves using reduced dimensionless terms, see Figure 6.3 where we use $\phi_{c}=0.56$ and $\phi_{m}=0.47$. The the flow curves are "S-shaped", where at a critical shear stress the suspension begins to shear thicken from one Newtonian regime until it plateaus at a higher viscosity at a second Newtonian regime, except for the higher volume fractions which tend to infinity.

Figure 6.2 a) illustrates the concept. Consider suspension A which has a comparatively low $\phi$. At vanishing shear stress, suspension A will have a viscosity given by the lower (red) curve, diverging at the friction-less $\phi_{c}$. Increasing the shear stress causes the viscosity to increase (vertical arrow) to a point on the dashed line in the middle of the extremes. The exact position of the dashed curve is determined by the fraction of particles in frictional contact at the imposed stress (see inset), in turn setting the divergence point $\phi_{J}\left(\sigma_{r}\right)$. A further increase in stress eventually causes the viscosity to plateau at the limiting value corresponding to all particles being in frictional contact (blue line, diverging at frictional $\phi_{m}$ ). The stress dependent viscosity is plotted in Figure 6.2 b), where suspension A displays CST between the limiting viscosity values correspond to all friction-less or all frictional particles. Suspension B (higher $\phi$ ) experiences a much steeper increase in viscosity, plateauing at a frictional viscosity orders of magnitude higher than the frictionless value on account of its closer proximity to the frictional divergence. We are now in the DST regime. Suspension C to start with has a $\phi$ that exceeds the frictional divergence $\phi_{m}$. While unproblematic in the friction-less state, once driven past the shear thickening transition, the viscosity diverges rapidly as the limiting value for all frictional contacts is already passed. At this point the suspension is in a fully jammed state.

Wyart-Cates define DST to be occurring when $\frac{d \dot{\gamma}}{d \sigma}=0$. Interestingly, the model first reaches this condition, and therefore predicts DST behaviour, at $\phi_{D S T} \approx 0.53$ which is notably lower than frictional jamming fraction we used of $\phi_{m}=0.47$. At $\phi_{m}$ and above is where the viscosity tends to infinity at the critical stress, indicative of a completely jammed frictional state. This forms three phases in the model, a continuously shear thickening phase, a discontinuously shear thickening phase, and a jammed phase. 

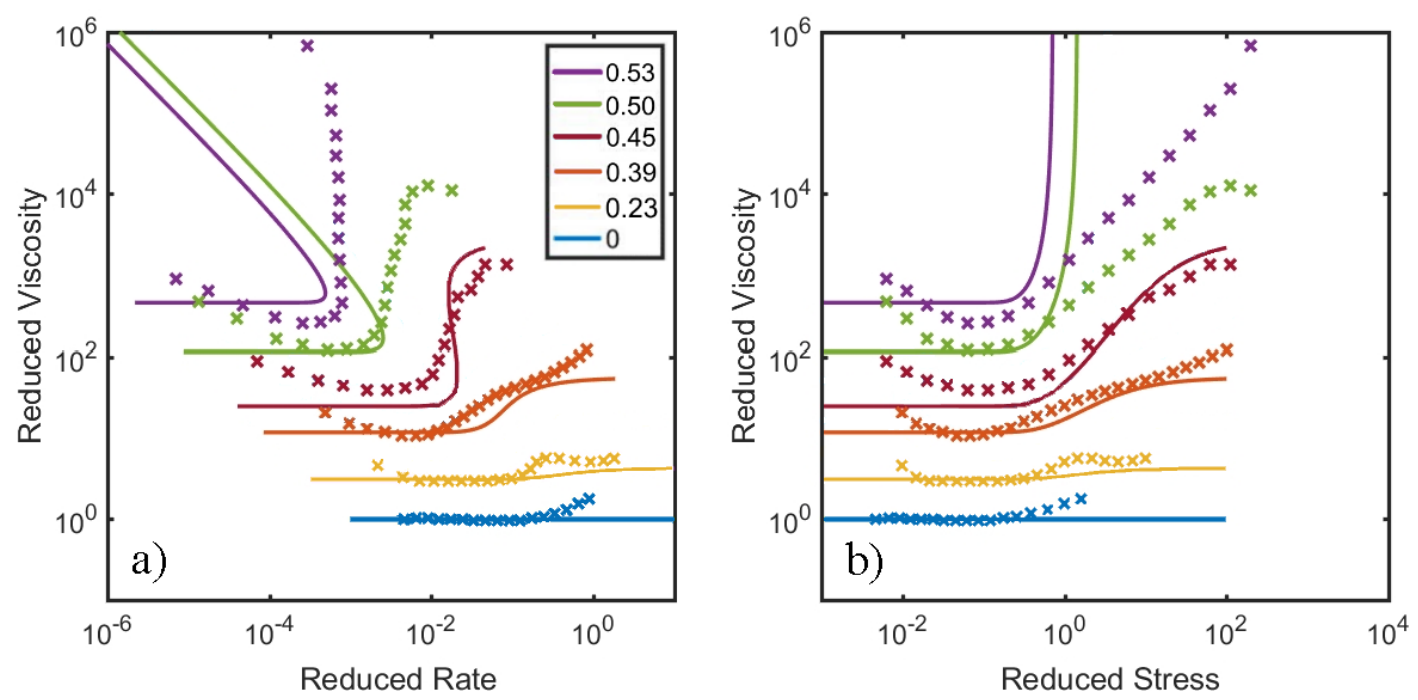

Figure 6.3: Dimensionless rheological curves produced using Wyart-Cates model with $\phi_{c}=0.56$ and $\phi_{m}=0.47$ compared with dimensionless rheological measurements obtained from Figure 6.1 .

\subsubsection{Going Dimensionless}

To compare the theory with our measurements directly, we need to reduce our rheological measurements to dimensionless numbers in the same fashion as the Wyart-Cates model. To do so, a critical shear stress, $\sigma_{c}$ and an interstitial fluid viscosity, $\eta_{s}$ are needed.

The interstitial fluid viscosity, $\eta_{s}$ is simply the viscosity of water at $25 \mathrm{C}^{\circ}$, $\eta_{s}=9 \mathrm{mPa} . \mathrm{s}$. Given that the critical shear stress at which shear thickening first occurs in cornstarch suspensions is approximately independent of $\phi$, it seems reasonable to assume this is the critical stress that needs to be overcome for frictional contacts to be made. However as mentioned, it is known that the geometry of the system can influence $\sigma_{c}$ [115, 121], as can be seen in our measurements where the higher five concentrations used a taller gap spacing to minimise confinement effects during readings and have a higher critical stress $\left(\sigma_{c}=0.5 \pm 0.1 \mathrm{~Pa}\right)$ than the lower five concentrations $\left(\sigma_{c}=0.1 \pm 0.1 \mathrm{~Pa}\right)$.

It follows then that our measured viscosity can be divided by our $\eta_{s}$, our measured shear stresses can be divided by our $\sigma_{c}$, and our measured shear rates can be divided by $\dot{\gamma}_{c}=\frac{\sigma_{c}}{\eta_{s}}$ to find the reduced dimensionless values that can be compared with the theory. See Figure 6.3 which used a scaling factor of $c=10$ in Equation 6.8 to obtain flow curves similar to our measurements. 


\subsubsection{Comparison}

The model is most sensitive to volume fraction, Figure 6.3 illustrates that the measured dimensionless rheological data collapses well onto the theoretical flow curves for some regimes. Each measured volume fraction has a critical stress at which thickening occurs that overlaps with the theoretical thickening behaviour. The four higher measured volume fractions each have similar viscosities to the theoretical curves for stresses immediately prior to thickening. Where as the four lower measured volume fractions have similar viscosities to the theoretical curves for stresses during thickening. Of course, water has a reduced Newtonian viscosity of 1 for all stresses, excluding the erroneous thickening at high shear rates discussed above.

A few things should be noted about the shape of the curves predicted by the theory when compared with rheological readings of cornstarch suspensions. First, the curves do not exhibit any shear thinning, which is well documented in cornstarch suspensions and present in our measurements [210, 211]. It has been proposed that the theory does not account for the observed shear thinning behaviour as the small yield stress associated with cornstarch suspensions is the result of soft-particle interactions which the theory does not account for, it models cornstarch particles as hard spheres [125].

Second, the shear rate for the theoretical curves exhibit a negative gradient as it begins to DST. This effect is slightly noticeable in our highest volume fraction but has not yet been recorded in experiment to the extent that the theory predicts [121, 125]. Hermes, Wyart, and Cates (2016) [125] investigated this discrepancy and found that the modelled behaviour is based on the premise that the sample maintains a steady flow, however the practical measurements allow for unsteady flows. They suggested that due to the parallel plate geometry the localised particles that first exhibit the DST effect at the outer boundary of the suspension (where the shear stress is highest) could migrate towards the centre of the sample (where the stress is lowest) relieving the DST response and allowing flow to continue. This particle migration effect also explains why a pool of cornstarch suspension can support a weight upon its surface only briefly [212]. Thus the theoretical negative gradient was not significantly observed in Hermes et al. rheology or in the results in this thesis as the DST response was "smoothed" by the particle migration in an unsteady flow. 
Third, the theoretical flow curves with volume fractions closest to RCP follow a negative gradient until the shear rate becomes zero, indicating a completely jammed state that would prevent the rheometer from continuing to rotates; this is also not observed in measurements. The model successfully predicts that no steady flow will occur for these dense suspensions above a critical shear stress but does not account for unsteady flow phenomenon such as the particle migration, fracturing, and slip that allows for the rheometer to impose a shear rate upon the suspension [125, 146]. Of course, this transition from a steady flow to an unsteady flow phenomenon like fracturing is precisely the focus of this thesis and will be investigated in the following chapter.

\subsection{Conclusion}

Parallel plate rheometry was used with a range of imposed stresses that spanned five orders of magnitude to analyse the shear thickening response of varying concentrations of cornstarch in water suspension. These measurements were then dimensionlessly compared with theoretical flow curves produced using Wyart-Cates model of shear thickening in particle suspensions. The model describes the suspension as in a particle-frictionless state at low stresses and transitions to a particle-frictional state at a critical stress that overcomes a particle repulsive force, increasing its viscosity. This model produces three regimes of behaviour with increasing filling fraction of particles in suspension, CST (transitionary curve of $\frac{d \dot{\gamma}}{d \sigma}>0$ ), DST (transitionary curve of $\frac{d \dot{\gamma}}{d \sigma}>0$ ), and jammed (curve never transitions to higher steady viscosity).

In agreement with previous studies [110, 115, 119, 121, 125], the cornstarch suspensions were found to transition between two Newtonian regime viscosities as a function of shear stress. Suspensions of $\phi \leq 0.41$ transitioned with CST, suspensions of $\phi \leq 0.50$ transitioned with DST, while those with $\phi \geq 0.53$ jammed and did not transition to a second Newtonian regime. As reported in literature, these transitions occur at a similar critical shear stress that is independent of $\phi$ but dependant on the rheometer geometry [115, 121].

The Wyart-Cates model was found to predict the general trends of CST and DST flow curves as function of both $\phi$ and $\sigma$ quite accurately. The theoretical flow curves transition at a critical reduced shear stress and had similar reduced viscosities leading up to that point, excepting that the model does not include the shear thinning observed at low stresses. The reduced viscosity after transition was also agreeable for the less dense concentrations that exhibited CST behaviour. 
However, the main discrepancy is found after DST behaviour begins for the denser suspensions. The model predicts the initiation of DST well but the flow curves do not account for non-steady flows that will occur in real systems, including particle migration and fracturing. This results in the true DST and jamming behaviour of cornstarch solutions in this geometry appearing where the model predicts but not being quite as extreme as the model suggests. This finding is in line with a previous publications that also rheologically tested Wyart-Cates theory with cornstarch suspensions [121, 125].

This theory was presented, along with rheological work produced to test it, as it gives a framework for what can be expected of DST suspensions in other experimental setups. The subject of this thesis is the transition from flow to fracture patterns in multiphase flow. This model and these measurements predict different regimes of behaviour for shear thickening fluids of different concentrations, some of which include non-steady flow patterns with particle migration and jammed states that can induce fracturing. Of particular interest is the fact that the concentrations in DST and jamming regimes have a low stress state for which steady flow is possible, from where the extreme solid-like behaviour transitions from. In the following chapter the different regimes will be visually explored in Hele-Shaw cell pattern analysis within the context of Wyart-Cates theory. 


\section{Chapter 7}

\section{Fracturing in a Discontinuous Shear Thickening Fluid}

\subsection{Introduction}

Recent theoretical and experimental work suggests a frictionless-frictional transition with increasing inter-particle pressure explains the extreme solid-like response of DST suspensions. However, analysis of macroscopic DST behaviour and flows in geometries other than the standard rheometry tools remain scarce. Here we study the displacement by pressurized air of a shear-thickening cornstarch suspension confined in a Hele-Shaw cell. We find three distinct flow patterns: viscous fingering, dendritic fracturing, and large-scale fracturing. We relate the Hele-Shaw flows to flow curves obtained by rheometry, and show that the transitions between the observed flow patterns can be explained as a dynamic transition between weak continuous shear thickening (CST), discontinuous shear thickening (DST), and complete jamming of the granular suspension in the framework of a recent theoretical model discussed in the previous chapter proposed by Wyart and Cates (2014) [146].

The experiment in this chapter was designed, performed, and analysed by myself with the supervision of Bjørnar Sandnes.

\subsection{Experimental Method}

The two controlled variables for experiments were the volume fraction of the cornstarch in suspension, ranging from $\phi=0.23 \pm 0.01$ to $0.53 \pm 0.01$, and the imposed air pressure, ranging from $P=0.10 \pm 0.02$ bar to $10.00 \pm 0.02$ bar. For reference, the unstressed critical jamming volume fraction of cornstarch is estimated as $\phi_{c}=0.56$ [118]. The cornstarch was first prepared in a vacuum chamber for 45 minutes to remove moisture from the sample to avoid ambient humidity influencing the volume fraction calculations. The dried sample was then weighed out and mixed with deionised water to form the desired 
volume fraction $\phi$ according to the following ratio:

$$
\phi=\frac{m_{c s} / \rho_{c s}}{m_{c s} / \rho_{c s}+m_{w} / \rho_{w}}(1+\lambda),
$$

where $m_{c s}$ is the measured mass of cornstarch, $\rho_{c s}$ is the density of cornstarch $(1.6 \pm$ $0.05 \times 10^{3} \mathrm{~kg} . \mathrm{m}^{-3}$ [114, 122, 151]), $m_{w}$ and $\rho_{w}$ is the mass and density of deionised water respectively, and $\lambda$ the fraction of cornstarch volume increase caused by swelling when submerged in water $(\lambda \approx 0.3[123])$. The prepared sample was stored in a sealed container to avoid evaporation before it was used. The experiment was run within minutes of the mixture being poured into the cell to avoid settling, which takes place over several hours even without a density matched liquid [114]. The particles of cornstarch were measured with a Mastersizer to have an average diameter of $16.1 \mu \mathrm{m}$ in the water suspension, with $90 \%$ of particles within the $10-25 \mu \mathrm{m}$ diameter range (see Figure 6.1 in the previous Chapter).

The experiments were conducted in a Hele-Shaw cell made of transparent $4 \mathrm{~cm}$ thick acrylic plates, see Figure 7.1 b). The cornstarch suspension was confined in a $0.02 \mathrm{~cm}$ gap underneath a removable upper circular plate with a $10 \mathrm{~cm}$ radius. The cell was held together by clamps and had a completely open boundary at its perimeter. Pressurized air entered the cell through a central $0.5 \mathrm{~cm}$ diameter inlet. Air was pre-pressurised in a $3.5 \mathrm{~L}$ metal cylinder to the desired pressure and then released with a valve into the inlet to begin the experiment.

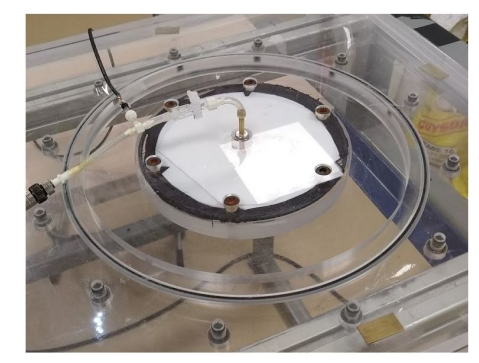

A)

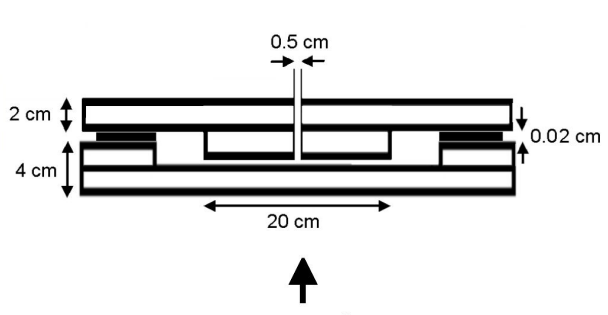

B)

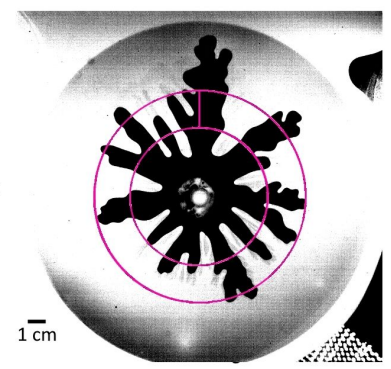

C)

Figure 7.1: Experimental setup. A) Photograph of circular Hele-Shaw cell used. B) side-view schematic of Hele-Shaw call design; all lengths are measured within $\pm 0.005 \mathrm{~cm}$. C) Example raw-image from an experiment using $\phi=0.23$ of cornstarch suspension subject to an invasion from the central inlet of air pre-pressurised to 0.1 bar. The $2 \mathrm{~cm}$ wide pink annulus $4 \mathrm{~cm}$ from the inlet represents the area analysed for each experiment. 
Invasion patterns were filmed from below with a high-speed camera (Photron Fastcam 1024-PCI) with a resolution of $1024 \times 1024$ pixel $^{2}$ and a frame rate of 1000 fps. Pattern characterization was performed on selected frames where the most advanced finger (or fracture branch) had reached to within $1 \mathrm{~cm}$ of the open boundary. Thresholding and de-speckling produced the binary images presented here. Morphology measurements were applied to a $2 \mathrm{~cm}$ wide annulus with inner radius $4 \mathrm{~cm}$ as illustrated in Figure $7.1 \mathrm{c}$ ). Finger (branch) width $w$ and separation distance between them $d$ was measured with intersecting 1 pixel wide rings and averaged within the annular region of interest. The spatial density of fingers (branches) was defined as $D=(w+d)^{-1}$. A characteristic finger (branch) tip velocity $v_{t i p}$ was estimated by measuring the time taken from when the air first entered the cell until the first branch reached the $10 \mathrm{~cm}$ radius boundary of the cell.

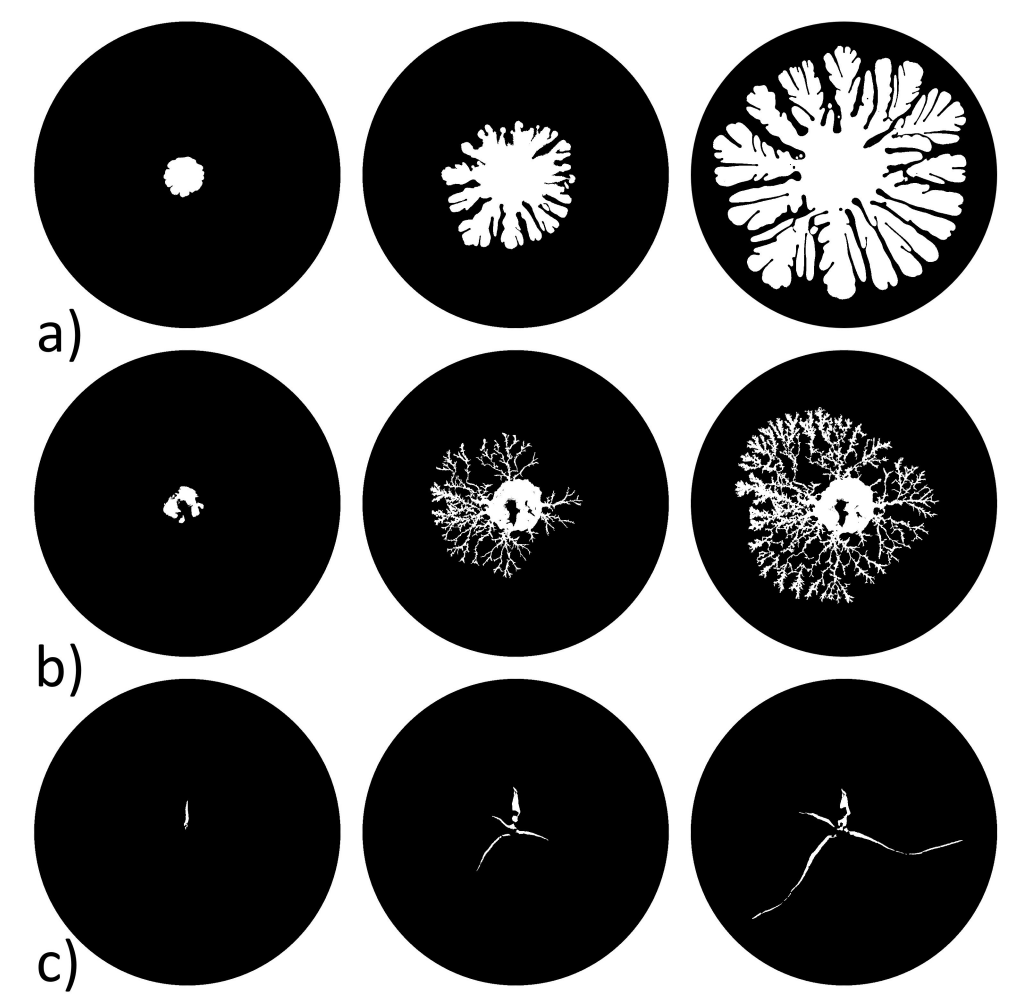

Figure 7.2: Pattern formation of pressurised air (white) invading cornstarch suspension (black). a) Viscous fingering (VF): $\phi=0.34, P=0.5$ bar with images at $0.015 \mathrm{~s}, 0.045 \mathrm{~s}$, and $0.090 \mathrm{~s}$ after air released. b) Dendritic fracturing (DFr): $\phi=0.47, P=5$ bar at $0.005 \mathrm{~s}, 0.015 \mathrm{~s}$ and $0.020 \mathrm{~s}$. c) Large fracture (Fr): $\phi=0.53$, $P=1$ bar at $0.270 \mathrm{~s}, 0.720 \mathrm{~s}$ and $1.020 \mathrm{~s}$. Image diameters correspond to the cell diameter $(20 \mathrm{~cm})$. 
a)
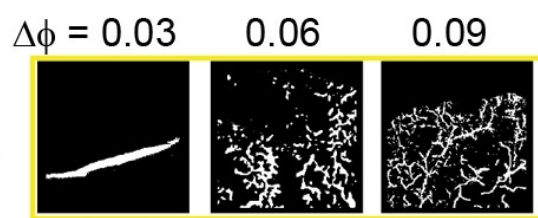

0.13

0.16

0.19

0.25

$P=$

1 bar
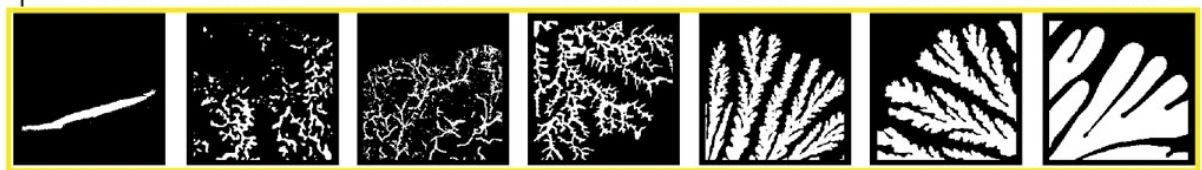

b)

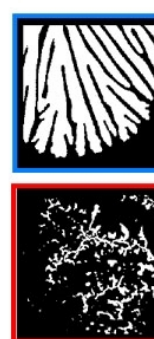

$P$ (bar)

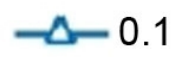

ह c)
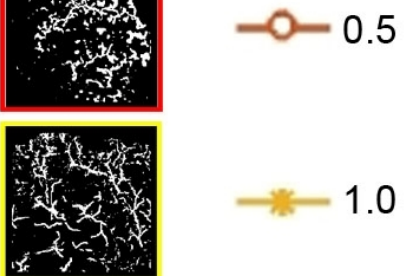

过

c)
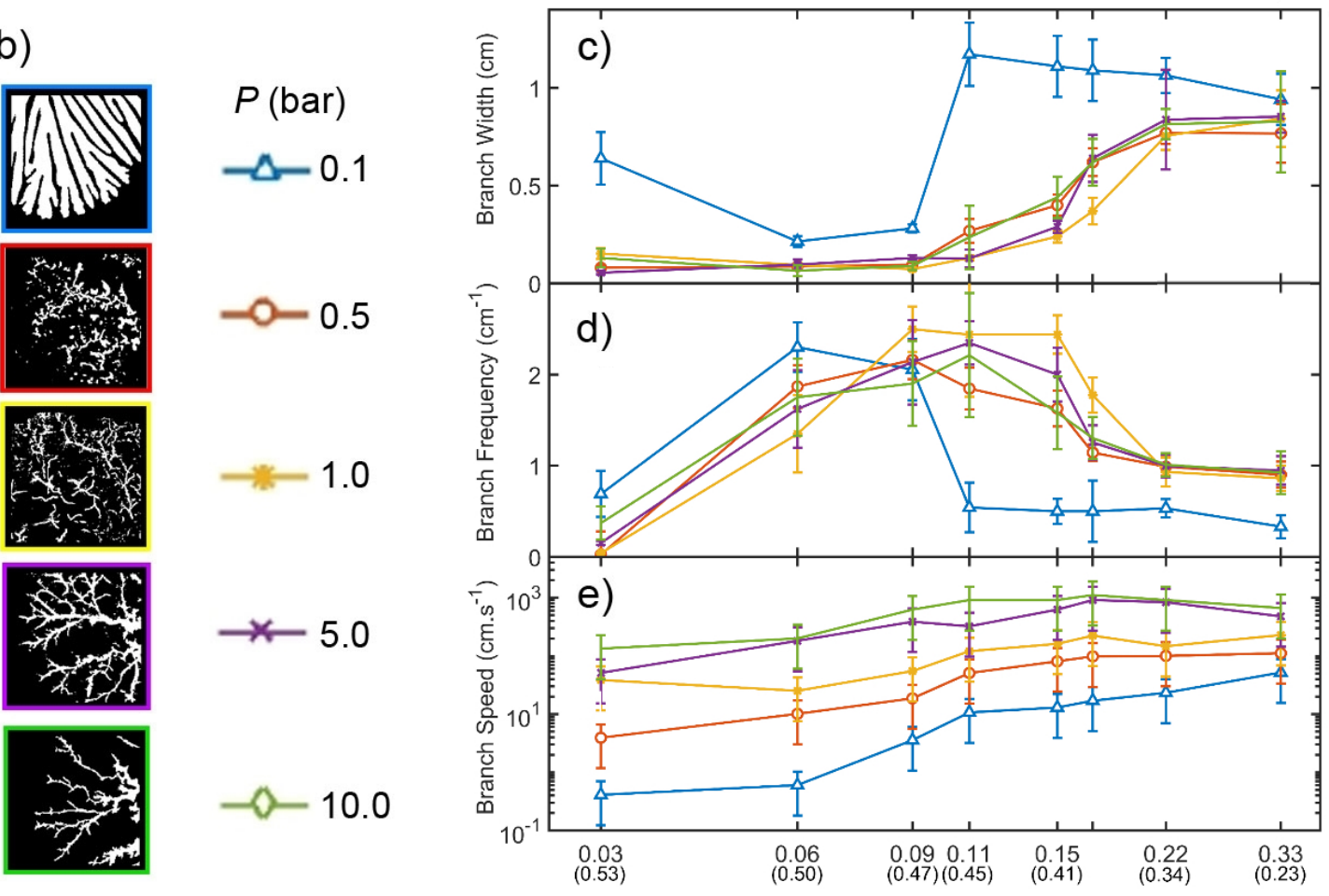

$\Delta \phi=0.09$

$\Delta \phi$

Figure 7.3: Pattern characteristics. a) $4 \times 4 \mathrm{~cm}$ tiles showing pattern morphology in experiments with varying $\phi$ and the air pressure kept constant $(P=1$ bar). Densest suspension to the left $(\Delta \phi=0.03)$, most dilute suspension to the right $(\Delta \phi=0.25)$. b) $4 \times 4 \mathrm{~cm}$ tiles showing pattern morphology in experiments with varying pressure and $\phi$ constant $(\Delta \phi=0.09, \phi=0.47)$. c) - e) shows branch width $w$, spatial density $D$, and tip velocity $v_{t i p}$, respectively, as a function of $\Delta \phi . w$ distinguishes whether the morphology is fingering (larger width) or fracturing. $D$ is inversely proportional to the separation distance between neighboring branches, and distinguishes whether the fracturing is dendritic (high branch frequency) or system-wide (low branch frequency). $v_{t i p}$ increases for higher imposed pressures and for more dilute suspensions (lower effective viscosity). Note that there is no step change in the tip velocity at the transition between fingering and fracturing. 


\subsection{Results and Discussion}

\subsubsection{Hele-Shaw Flow and Pattern Formation}

During an experiment the pressurised air enters the circular cell through a central inlet and pushes the cornstarch suspension aside as it invades into the cell gap. This suspension displacement forms the observed radial patterns, see Figure 7.2, and the experiment ends once the pressurised air reaches the edge of the cell and is released to atmosphere. We have identified three distinct pattern morphologies in our experiments: viscous fingering (VF), dendritic fracturing (DFr), and large, system-wide fractures (Fr) as displayed in Figure 7.2 a)-c) respectively. Figure 7.3 a) shows a more detailed view: $4 \times 4 \mathrm{~cm}$ closeups of representative patterns obtained in experiments with different volume fraction $\phi$ at $P=1$ bar. It is useful to represent the volume fraction in terms of how far the system is from the unstressed critical jamming volume fraction $\Delta \phi=\phi_{c}-\phi$. Figure 7.3 shows measured pattern characteristics plotted against $\Delta \phi:$ c) branch/finger width $w$, d) spatial frequency of branches/fingers $D$, and e) branch/finger tip velocity $v_{t i p}$.

Dilute suspensions (see e.g. Fig. 7.2 a and Fig. 7.3 a $\Delta \phi=0.25$ ) produce viscous fingering (VF) with smooth curvature at the finger tips. Dendritic fractures (DFr) at intermediate $\Delta \phi$ (Fig.7.2p and Fig. $7.3 \mathrm{~b}$ and a $\Delta \phi=0.06-0.13)$ are distinct from VF by having significantly narrower branch widths $w$ (Fig. $7.3 \mathrm{p}$ ). The narrow DFr branches grow by frequent tip-splitting, giving rise to spatially dense fracture networks (high $D$, see Fig. 7.3 c). The DFr network spreads radially outwards from the injection point.

The DFr mode displays an interesting dynamic whereby the suspension is momentarily forced into a DST solid-like state and moments later relaxes back to a fluid state after the passage of the invasion front. Figure 7.4 a)-c) shows a time-series of close-up images where the suspension of $\phi=0.50$ is invaded by air of $\mathrm{P}=0.3$ bar from the lower left hand corner. The suspension is sheared by the oncoming invasion front into a DST state that subsequently fractures into thin (sub-mm), jagged branches. After the fracture front has passed, the shear stress on the suspension decreases, and the suspension relaxes back to a liquid state. Surface tension then acts to smooth the interface and widen the branches. Figure 7.4 d) shows the branch width $w$ measured at different radial distances as a function of time for the time-series, illustrating the continued widening of the branches after the passage of the solidification/fracture front. Relaxation behaviour on the scale of seconds in dense cornstarch suspensions has been reported on previously and linked with the time taken for local particles in contact to flow way from each other [116, 118].

The densest suspension (Fig. 7.2 $\mathrm{k}$ and Fig. 7.3a $\Delta \phi=0.03$ ) displays a very different fracturing pattern. Here, the suspension in the entire cell appears to jam up, and the air invades in long, uninterrupted branches that span the radius of the cell. The large fracture mode (Fr) is distinguished from the dendritic fractures (DFr) by having a much lower spatial fracture density $D$ (Fig. 7.3 c). 
There is no abrupt change in the tip velocity as the pattern transitions from fingering to fracturing. The general trend (Fig. $7.3 \mathrm{~d}$ ) is simply increased velocity for higher imposed pressures and for more dilute suspensions (with lower effective viscosity).

\subsubsection{Discussion}

Figure 7.5 a) shows selected experimental patterns in a phase diagram with air pressure $P$ versus filling fraction difference $\Delta \phi$ in a log-log plot. The colourmap represents measurements of b) branch width $w$ in blue, c) spatial frequency $D$ in red, and d) tip velocity $v_{\text {tip }}$ in green, obtained by normalising the data with the respective maximal values as presented in Figure 7.3 b)-d). The phase diagram combines these measurements into a RGB colour space interpolated between a total of 40 experiments spanning the $P-\Delta \phi$ space. The three main patterns are thus grouped by the measured characteristics: Large fractures (Fr) for the densest suspensions in a green hue, Dendritic fracturing (DFr) for intermediate $\Delta \phi$ and $P>0.5$ bar in yellow-orange, and viscous fingering (VF) in blue-pink-purple hues corresponding to dilute suspensions and low imposed air pressure.

To model the observed transitions in flow behaviour we adopt a theory recently developed by Wyart and Cates (2014) outlined in the previous chapter. At the heart of the theory is the hypothesis proposed by several authors that DST is caused by a stress-induced

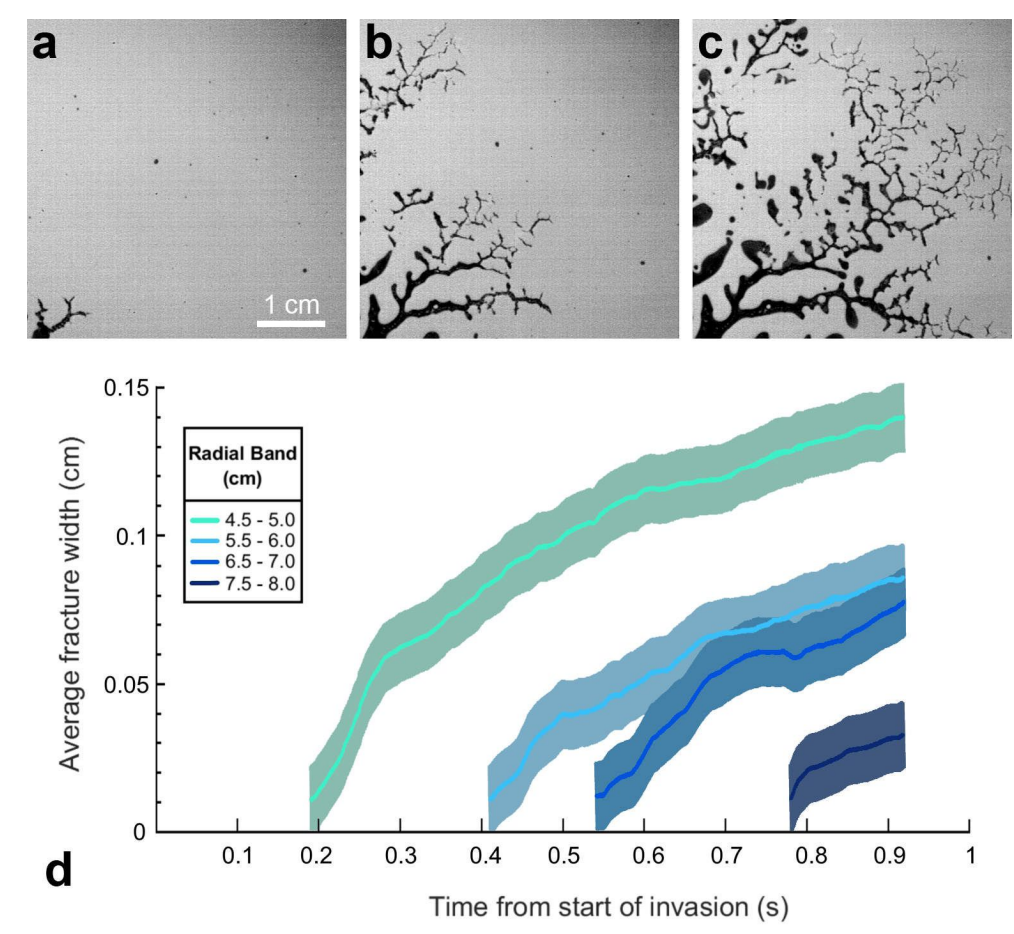

Figure 7.4: Dendritic fracturing at $\phi=0.50$ and $\mathrm{P}=0.3$ bar which straddles the VF-DFr regime transition. a)-c) shows close-up of dendritic fracturing at 0.2, 0.5, and $0.8 \mathrm{~s}$ respectively, where the invading fracture front temporarily forces the suspension into a DST state which relaxes back to a liquid state after the front has passed. d) shows that the fractures continue to widen as surface tension acts on the interface after the front has passed. 

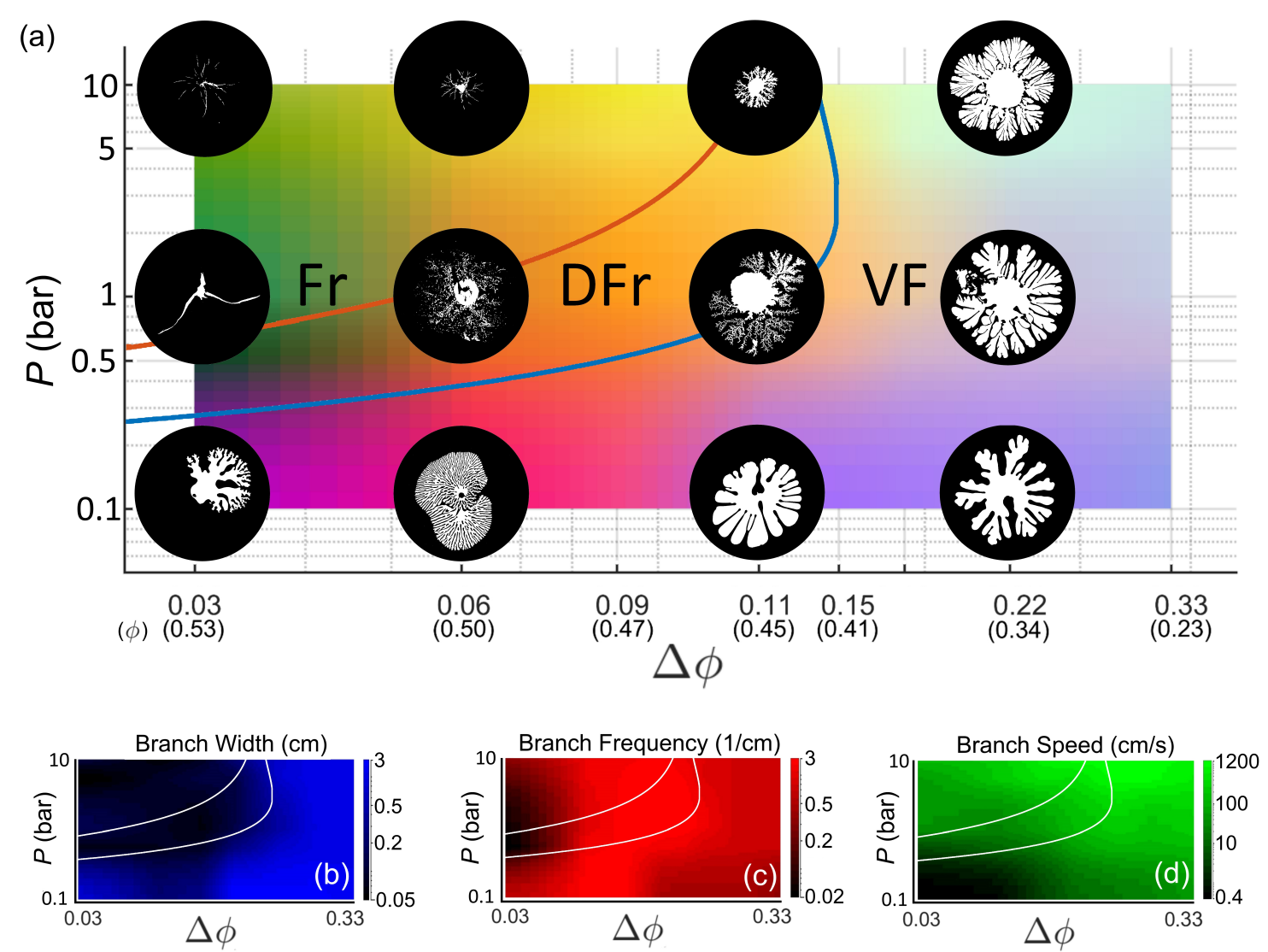

Figure 7.5: a) Sample of patterns formed at varying invasion pressures and volume fractions of cornstarch presented in a logarithmic plot. The volume fractions are presented as a $\Delta \phi$, the difference between the critical jamming fraction of 0.56 and the volume fraction $\phi$. The experiments are all to scale with the radius of their image equivalent to $10 \mathrm{~cm}$. Coloured background plot represents the analysed data from the experiments in a RGB colour space, where b) is the green colour space for the normalised logarithmic branch speed, c) is the blue colour space for the normalised logarithmic branch width, and d) is the red colour space for the normalised logarithmic branch frequency. Similar pattern morphologies are evident by groups of similar colour. Three phases are noted, fracturing, dendritic fracturing, and viscous fingering. The transitionary lines predicted by Wyart-Cates theory are displayed, approximating the experimental transitions between these phases.

transition from friction-less to frictional inter-particle interactions [125, 127, 143-146].

$$
\frac{\dot{\gamma}_{r}}{\sigma_{r}}=\left(1-\frac{\phi}{\phi_{m} f\left(\sigma_{r}\right)+\phi_{c}\left(1-f\left(\sigma_{r}\right)\right)}\right)^{2} .
$$


We note that recent advancements in the detailing of this theory have been made by including dilation effects for the viscosity divergence and more precisely modelling the frictional behaviour function "f" by including lubrication forces and modelling force chain buckling which both reduce frictional contact [125, 127, 159, 213]. However, we employ the simpler fundamental arguments laid out for the model by Wyart-Cates [146] to illustrate how our non-rheological data can be compared with the general predictions for DST behaviour made by this frictional-model.

Using the model, three states can be defined: CST, DST, and fully jammed, depending on suspension $\phi$ and stress $P$. Following Wyart and Cates [146] we adopt the following criteria for the transition between these states: CST to DST when $\mathrm{d} \eta / \mathrm{d} \sigma=1$, and DST to fully jammed when $\mathrm{d} \eta / \mathrm{d} \sigma=\infty$. The locus of points satisfying these two conditions are plotted as a function of $\phi$ in Fig. 6.2 c) in the previous Chapter. These two phase boundaries are superimposed on the phase diagram in Fig. 7.5 as a function of $\Delta \phi$ (rather than $\phi$ ). We indirectly relate the reduced stress $\sigma_{r}$ from the theoretical model to the Hele-Shaw experiment by setting $\sigma_{c}$ to correspond to the minimum pressure producing fracturing in the minimal $\phi$ that fractures ( $P_{c} \approx 1$ bar using $\phi=0.45, \Delta \phi=0.13$ ).

The transition from continuous to discontinuous shear thickening as predicted from the theory (blue line in Fig. $7.5 \mathrm{k}$ ) is seen to correspond to the observed transition from viscous fingering (VF) to dendritic fracturing (DFr) in the Hele-Shaw experiments. We associate the theoretical DST to jammed transition (red line) with the observed change from small scale DFr to system-spanning fractures (Fr), although the experimentally observed Fr mode (green hue in phase diagram) is only seen for the most dense suspensions ( small $\Delta \phi)$.

We put forward the following interpretation: The viscous fingering patterns are a signature of liquid-like behaviour in the suspension. In the context of the measured rheology and the theoretical model, this liquid-like behaviour occurs at low stress or when the suspension is dilute enough that only weak (continuous) shear thickening can be obtained regardless of imposed stress. Our results are in agreement with the central hypothesis put forward by Wyart and Cates and several other authors [125, 127, 143-146], that DST is a signature of a friction-less to frictional transition, where the friction-less state is maintained by an inter-particle repulsive force. This is in particular revealed by the fact that the VF mode, i.e. liquid-like behaviour, was observed for even the highest concentrations as long as the imposed pressure was below the critical stress. 
We propose that the fracturing mode is a signature of solid-like behaviour which in turn is a consequence of frictional inter-particle contact, as similarly observed in other multi-phase granular systems [214, 215]. This mode is associated with the order-ofmagnitude increase in stress for a small increase in shear rate, i.e. DST, as evident in the measured and theoretical flow curves. For the dendritic fracturing mode (DFr), the suspension is locally brought into the frictional DST state around the invasion front, but the suspension relaxes back to its liquid state as the stress reduces once the front has passed by. We have here used $\mathrm{d} \eta / \mathrm{d} \sigma=1$ criterion for the continuous to discontinuous shear thickening transition, but we note that this choice is somewhat arbitrary, and other criteria could also be proposed [146].

The theoretical model features a distinct transition from DST to fully jammed corresponding to the point at which the initial $\phi$ for the quiescent friction-less suspension is above that of the maximum $\phi_{m}$ for frictional particles. This theoretical result can be associated with our measured rheology: it occurs for $\phi \gtrsim 0.6$ when the flow curve no longer levels off at a well-defined upper viscosity branch. We suggest that this dynamic is evident in the Hele-Shaw experiments for similar values of $\phi$ where the stress imposed by the invading air causes a large fraction of the suspension contained in the cell to seize up in a solid-like state, resulting in large, system-spanning fractures.

\subsection{Conclusion}

Here we have reported on the first use of a Hele-Shaw cell geometry to visualise invasion patterns in shear thickening and DST cornstarch suspensions. Air was released at a controlled pressure into suspensions with varying concentration of cornstarch and the resultant invasion patterns were captured with a high-speed camera. Results from image analysis lead to a delineation between three distinct pattern morphologies: conventional viscous fingering, dendritic fracturing marked by dense frequently branching fractures, and system-wide fracturing marked by sparse minimally branching fractures. These results were plotted in a $\Delta \phi$-P phase diagram and explained in context of rheological measurements as well as phase transitions predicted by Wyart-Cates frictionless-frictional DST theory. 
We found that the striking invasion patterns revealed by the alternate flow geometry of the Hele-Shaw cell correspond with the three different rheological responses of cornstarch suspensions described by frictional-frictionless theory. This represents an example of frictionless-frictional theory providing quantitative explanatory power for nonrheometric experimental data. Viscous fingering was observed during conditions that did not correspond to DST or jamming (CST for dilute suspension and $P<P_{c}$ for all suspensions). Dendritic fracturing was observed during conditions that correspond to DST behaviour but not jamming $\left(P>P_{c}\right.$ for dense suspensions of $\left.\phi<\phi_{m}\right)$. And systemwide fracturing was observed during conditions that correspond to jamming $\left(P>P_{c}\right.$ for $\left.\phi>\phi_{m}\right)$.

In addition, a DST relaxation phenomenon is reported in the dendritic fracturing regime. The advancing local stress front caused by the invading air moves through the suspension initially as fractures. However, once the front has passed, the local stress is lowered and the fractures "relax" into fingers whilst the front continues to fracture ahead. This indicates that the dendritic fracturing regime is categorised by a local DST response, rather than a global one as the jammed regime does.

Future experimental work involving Hele-Shaw cell setups can explore how varying the cell gap height should change the shear stress experienced by the suspension relative to the imposed invasion pressure, as pursuing this could help illuminate how to directly relate the two. In addition, recent advancements in modelling dense DST suspensions based on frictionless-frictional theory have allowed complex mechanical responses of cornstarch suspensions to be simulated [127]. The simple geometry and distinct visual results presented here could be useful as a case-study to validate simulations. 


\section{Chapter 8}

\section{Conclusion}

\subsection{Objectives}

Chapter 1 laid out the desire of this thesis to further understand how a particular set of non-biological patterns self emerge in natural and engineered environments. The patterns in question are fracture-like cavities that can form within a constrained geometry as a liquid mixed with granular material (which in other circumstances can readily flow as a fluid) is subject to an invading gas. The questions raised included:

- For what conditions did a flowing to fracturing transition occur in a Hele-Shaw cell?

- What mechanics are causing the solid-like fractures to form?

- What parameters determine the resultant pattern characteristics?

These questions were directed at two granular materials that behave differently under similar circumstances. The first was cohesionless granular packings settled in water and the second was DST suspensions of cornstarch particles in water.

Chapter 2 and 3 placed the work performed in this thesis into context within the literature as it currently stands. There has been a bevy of work performed illustrating the wide variety of multiphase invasion patterns that can be observed in settled granular packings within Hele-Shaw cells, including fracturing. However, the behaviour of the fracturing regime had yet to be a focus of a significant amount of study. There has also been a lot of debate in literature regarding the mechanisms behind DST in cornstarch suspensions as well as experiments performed to examine its rheological properties. However, the utility of visualising invasion patterns within a Hele-Shaw cell had not previously been employed for a DST fluid. As such the questions asked in the outset of this thesis had yet to be adequately addressed for either system. 


\subsection{Fracture Patterns in Settled Grains}

In Chapter 4 the granular motion during fracture formation in settled multiphase HeleShaw invasion experiments was examined with a high-speed camera and PIV. It was shown that during a single fracturing event multiple sub-millimetre and microsecond events were occurring, with pauses in motion between each of them. Together these micro-events amalgamated into the one macro-scale observed fracture event.

Intermittency of fracturing events had previously been reported, but intermittency within a fracturing event was first reported here. The PIV tracking of granular motion revealed that the fractures formed as granular material was compacted ahead of and to either side of the opening cavity. In addition, the opening of fracture cavities generally occurred past narrow necks of grains that remained static, suggesting that the invading gas had to first pass through the pore spaces of a compacted region that was resistant to motion before expanding into a cavity on the other side. It was proposed that the higher capillary pressure required to invade these compacted regions is what generates the character length scale observed in the self-avoiding macro-scale fracture invasion patterns.

Building on Chapter 4, in Chapter 5 the macro-scale pattern characteristics and parameters that dictate them was studied for fracturing in settled multiphase Hele-Shaw invasion experiments with PIV and image analysis. In addition, a transition from frictional fracturing to viscous flow was observed as a function of injection rate. It was determined that the frictional resistance between the grains and the cell, holding back the capillary stress exerted by the gas on the fracture interface, is what causes the jammed compaction fronts and the intermittency to manifest. The thickness of these compaction fronts controls the maximum density of the fracturing pattern. In concordance with our theory, it was shown that irregular grains with a higher coefficient of friction produced denser patterns than spherical grains. An equation was derived that predicts the density of fracture patterns based on the granular and cell properties of the system that agreed with experimental results. Lastly, the transition from fracturing to flowing was categorised with a dimensionless fluidisation number that determined at what injection rate the viscous forces from the flow of the displaced interstitial fluid would be high enough to overcome the friction of the granular packing. This fluidised regime was marked by system-wide motion of grains towards and out of the open boundary. 


\subsection{Fracture Patterns in DST Suspensions}

Chapter 6 consisted of supportive and exploratory work for Chapter 7. The different concentrations of cornstarch suspension that are used in Chapter 7 where subject to tests in a parallel plate rheometer so that their behaviour in Hele-Shaw cell experiments could be put into a rheological context. The resultant flow curves where also then compared with the theoretical flow curves produced by the Wyart-Cates model for DST in dense granular suspensions. This model states that the sudden increase in viscosity is a result of external stress overcoming inter-particle repulsion causing frictional particle contact. The theory was found to accurately predict the critical shear stress at which thickening occurs as well as which concentrations would CST, DST or jam. Discrepancies between the theoretical curves and the measured curves were highlighted and discussed. However, as the model has strong explanatory power with regards to the regimes and transitions of the cornstarch rheology it was decided that the experimental results in Chapter 7 would also be compared with it.

In Chapter 7 the invasion patterns formed in varying concentrations of cornstarch suspension when subject to pressurised air in a Hele-Shaw cell were examined with a high-speed camera and image analysis. Three pattern regimes were delineated from the analysis based on fracture branch width and density: viscous fingering, dendritic fracturing, and fracturing. Though fracturing invasion patterns had been recorded previously in viscoelastic fluids this is the first report of them in a DST fluid. It was found that the three regimes coincide with the CST, DST, and jammed conditions that can be described in a concentration vs. pressure phase diagram with transition conditions derived from Wyart-Cates theory. Importantly, it was observed that below a critical invasion pressure all of the concentrations evolved as viscous fingering and above that threshold the patterns transitioned to their respective regime. As such, these experiments support the theory that DST behaviour is caused by a frictionless to frictional transition, as viscous fingering is a liquid-like viscous phenomenon while granular fracturing is a solid-like frictional phenomenon. A specific limitation of this study is that the pressure of the invading gas could not be related to the shear stress experienced by the suspension in the Hele-Shaw geometry. Comparing the results in a dimensionless fashion circumnavigated this issue but it is a point that should be addressed in the future. 


\subsection{Comparing Systems}

Looking at both systems studied here, it appears that a common theme is how particle friction is critical for granular mixtures to fracture. It is friction that provides the necessary resistance in the defending mixture to support the rigid fracture patterns observed when a less viscous fluid is forced into a saturated deformable dense granular network. What is most interesting when comparing the two systems is that their behaviour can be considered to be inverted. The dense settled granular media has a critical injection pressure below which it fractures and above which it flows to the open boundary, whereas the dense suspended cornstarch has a critical injection pressure below which it always flows to the open boundary and above which it fractures. Of course, for both systems the transition is determined by when friction between particles is deactivated or activated. As the settled grains are resting in contact, friction is always present until the injection rate maintains at a high enough pressure that it can essentially suspend the particles; as the cornstarch particles rest in suspension avoiding contact, friction is never present until the injection pressure is high enough to force the particles into contact.

\subsection{Open Questions}

It would be curious to test if there is a second higher invasion pressure threshold above which viscous forces dominate the cornstarch suspensions again, analogous to fracture-toflow transition in settled granular media as well as the higher Newtonian regime reported in DST rheology. Similarly, another question posed by this work is that if the cornstarch particles are given enough time to settle prior to experiment will the system behave like that of the settled grains? Will the cornstarch fracture at low maintained pressures while the particles are settled, transition to flowing as the particles are suspended in higher pressures, and then transition to fracturing again when friction is reactivated at its critical shear stress. That cornstarch suspensions exhibit shear thinning at low stresses also suggest this might be possible. Given both untested scenarios, is it possible for DST suspensions to have fracture-flow-fracture-flow transitions with increasing stress?

It is also of great interest to see if these results will be replicated in a fully 3D system, as opposed to a Hele-Shaw cell which is significantly confined in one dimension. Are the resultant pattern structures also dominated by compaction fronts? Are fractures even formed or is the friction against the stiff, relatively local, confining cell critical to maintaining the rigid structures? Will the settled media transition to flowing only if there is enough pressure to suspend the whole system or can it flow locally? Will the DST suspension maintain a given fracture network under consistent invasion like the settled media or will it be dynamic with opening and closing branches?

Overall, the results laid out in this thesis incentivise a number of new avenues of enquiry for fracture and flow transitions in saturated granular media. 


\section{Bibliography}

[1] H. J. Spiers, N. Burgess, T. Hartley, F. Vargha-Khadem, and J. OKeefe, "Bilateral hippocampal pathology impairs topographical and episodic memory but not visual pattern matching," Hippocampus, vol. 11, no. 6, pp. 715-725, 2001.

[2] M. P. Mattson, "Superior pattern processing is the essence of the evolved human brain," Frontiers in Neuroscience, vol. 8, aug 2014.

[3] Aristotle, Metaphysics, vol. 1-5. 350 BC.

[4] L. da Vinci, The Notebooks of Leonardo da Vinci. No. p. 121, 1508.

[5] M. Livio, The Golden Ratio: The Story of PHI, the World's Most Astonishing Number. Crown/Archetype, 2008.

[6] A. Turing, "The chemical basis of morphogenesis," Philosophical Transactions of the Royal Society of London. Series B, Biological Sciences, vol. 237, pp. 37-72, aug 1952.

[7] P. K. M. T. E. Woolley, R. E. Baker, Turing's theory of morphogenesis. No. Chapter 34, Oxford University Press, 2017.

[8] I. Sumita and M. Manga, "Suspension rheology under oscillatory shear and its geophysical implications," Earth and Planetary Science Letters, vol. 269, pp. 468477, may 2008.

[9] J. Oppenheimer, A. C. Rust, K. V. Cashman, and B. Sandnes, "Gas migration regimes and outgassing in particle-rich suspensions," Front. Phys., vol. 3, no. 60, 2015.

[10] B. P. Boudreau, C. Algar, B. D. Johnson, I. Croudace, A. Reed, Y. Furukawa, K. M. Dorgan, P. A. Jumars, A. S. Grader, and B. S. Gardiner, "Bubble growth and rise in soft sediments," Geology, vol. 33, no. 6, p. 517, 2005.

[11] G. Varas, V. Vidal, and J.-C. Geminard, "Venting dynamics of an immersed granular layer," Phys. Rev. E, vol. 83, p. 011302, 2011. 
[12] A. J. Cavanagh and Haszeldine, "The sleipner storage site: Capillary flow modeling of a layered $\operatorname{co} 2$ plume requires fractured shale barriers within the utsira formation," Int. J. Greenh. Gas Control, vol. 21, pp. 101-112, 2014.

[13] A. Islam, S. Chevalier, I. B. Salem, Y. Bernabe, R. Juanes, and M. Sassi, "Characterization of the crossover from capillary invasion to viscous fingering to fracturing during drainage in a vertical 2D porous medium," Int. J. Multiphase Flow, vol. 58, pp. 279-291, 2014.

[14] C. M. Christiansen, C. Riis, S. B. Christensen, M. M. Broholm, A. G. Christensen, K. E. S. Klint, J. S. A. Wood, P. Bauer-Gottwein, and P. L. Bjerg, "Characterization and quantification of pneumatic fracturing effects at a clay till site," Environ. Sci. Technol., vol. 42, no. 2, pp. 570-576, 2008.

[15] U. Frank and B. N., "Remediation of low permeability subsurface formations by fracturing enhancement of soil vapor extraction," J. Hazard. Mater., vol. 40, pp. 191-201, 1995.

[16] S. Berg, H. Ott, S. A. Klapp, A. Schwing, R. Neiteler, N. Brussee, A. Makurat, L. Leu, F. Enzmann, J.-O. Schwarz, M. Kersten, S. Irvine, and M. Stampanoni, "Real-time 3d imaging of haines jumps in porous media flow," Proceedings of the National Academy of Sciences, vol. 110, pp. 3755-3759, feb 2013.

[17] H. S. Hele-Shaw, "On the motion of a viscous fluid between two parallel plates," Nature, vol. 58, pp. 34-36, 1898.

[18] P. G. Saffman and G. I. Taylor, "The penetration of a fluid into a porous medium or hele-shaw cell containing a more viscous liquid," Proceedings of the Royal Society of London. Series A. Mathematical and Physical Sciences, vol. 245, pp. 312-329, jun 1958.

[19] P. G. Saffman, "Viscous fingering in hele-shaw cells," Journal of Fluid Mechanics, vol. 173, p. 73, dec 1986.

[20] B. Sandnes, H. A. Knudsen, K. J. Måløy, and E. G. Flekkøy, "Labyrinth patterns in confined granular-fluid systems," Phys. Rev. Lett., vol. 99, no. 3, p. 038001, 2007.

[21] B. Sandnes, E. Flekkøy, H. Knudsen, K. Måløy, and H. See, "Patterns and flow in frictional fluid dynamics," Nature Communications, vol. 2, apr 2011.

[22] H. A. Knudsen, B. Sandnes, E. G. Flekkøy, and K. J. Måløy, "Granular labyrinth structures in confined geometries," Phys. Rev. E, vol. 77, no. 2, p. 021301, 2008. 
[23] B. Sandnes, E. G. Flekkøy, and K. J. Måløy, "Stick slip displacement of confined granular mixtures: bubble expansion,” Eur. Phys. J.-Special Topics, vol. 204, no. 1, pp. 19-25, 2012.

[24] R. Holtzman, M. L. Szulczewski, and R. Juanes, "Capillary fracturing in granular media," Phys. Rev. Lett., vol. 108, no. 26, p. 264504, 2012.

[25] G. Løvoll, Y. Méheust, R. Toussaint, J. Schmittbuhl, and K. J. Måløy, “Growth activity during fingering in a porous Hele-Shaw cell," Phys. Rev. E, vol. 70, no. 2, p. $026301,2004$.

[26] K. T. Tallakstad, H. A. Knudsen, T. Ramstad, G. Løvoll, K. J. Måløy, R. Toussaint, and E. G. Flekkøy, "Steady-state two-phase flow in porous media: statistics and transport properties," Phys. Rev. Lett., vol. 102, no. 7, p. 074502, 2009.

[27] A. Ferrari, J. Jimenez-Martinez, T. L. Borgne, Y. Méheust, and I. Lunati, "Challenges in modeling unstable two-phase flow experiments in porous micromodels," Water Resources Research, vol. 51, pp. 1381-1400, mar 2015.

[28] J. Kepler, Strena seu de nive sexangula. 1611.

[29] C. F. Gauss, Besprechung des Buchs von L. A. Seeber: Untersuchungen über die Eigenschaften der positiven ternären quadratischen Formen usw. 1831.

[30] G. D. Scott, "Packing of spheres," Nature, vol. 188, pp. 908-909, 1960.

[31] J. Bernal and J. Mason, "Co-ordination of randomly packed spheres," Nature, vol. 188, pp. 910-911, 1960.

[32] T. Aste, M. Saadatfar, and T. Senden, "Geometrical structure of disordered sphere packings," Physical Review E, vol. 71, jun 2005.

[33] M. Jerkins, M. Schröter, H. L. Swinney, T. J. Senden, M. Saadatfar, and T. Aste, "Onset of mechanical stability in random packings of frictional spheres," Physical Review Letters, vol. 101, jul 2008.

[34] G. W. Delaney, J. E. Hilton, and P. W. Cleary, "Defining random loose packing for nonspherical grains," Physical Review E, vol. 83, may 2011.

[35] H. Y. Sohn and C. Moreland, "The effect of particle size distribution on packing density," The Canadian Journal of Chemical Engineering, vol. 46, pp. 162-167, jun 1968 .

[36] K. W. Desmond and E. R. Weeks, "Influence of particle size distribution on random close packing of spheres," Physical Review E, vol. 90, aug 2014. 
[37] L. C. Verman and S. Banerjee, "Effect of container walls on packing density of particles," Nature, vol. 157, pp. 584-584, may 1946.

[38] K. W. Desmond and E. R. Weeks, "Random close packing of disks and spheres in confined geometries," Physical Review E, vol. 80, nov 2009.

[39] J.-F. Camenen, Y. Descantes, and P. Richard, "Effect of confinement on dense packings of rigid frictionless spheres and polyhedra," Physical Review E, vol. 86, dec 2012 .

[40] H. Darcy, "Les fontaines publiques de la ville de dijon," Paris: Dalmont, 1856.

[41] S. Whitaker, "Flow in porous media i: A theoretical derivation of darcys law," Transport in Porous Media, vol. 1, no. 1, pp. 3-25, 1986.

[42] M. Sahimi, "Flow phenomena in rocks: from continuum models to fractals, percolation, cellular automata, and simulated annealing," Reviews of Modern Physics, vol. 65, pp. 1393-1534, oct 1993.

[43] J. Kozeny, "Ueber kapillare leitung des wassers im boden," Sitzungsber Akad. Wiss., 1927.

[44] P. Carman, "Fluid flow through granular beds," Institution of Chemical Engineers, vol. 75, pp. S32-S48, dec 1937.

[45] A. C. Walshaw and D. A. Jobson, Mechanics of Fluids. Longmans, 1962.

[46] T. Young, "An essay on the cohesion of fluids.," Philosophical Transactions of the Royal Society of London, vol. 95, pp. 65-87, jan 1805.

[47] P. S. Laplace, Traité de Mécanique Céleste, vol. 4. Supplément au dixième livre du Traité de Mécanique Céleste, 1805.

[48] H. A. Janssen, "Versuche über getreidedruck in silozellen," Z. Ver. Dtsch. Ing., vol. 39, no. 35, pp. 1045-1049, 1895.

[49] B. Marks, B. Sandnes, G. Dumazer, J. A. Eriksen, and K. J. Måløy, “Compaction of granular material inside confined geometries," Front. Phys., vol. 3, p. 41, 2015.

[50] A. Einstein, "Eine neue bestimmung der moleküldimensionen," Annalen der Physik, vol. 324, no. 2, pp. 289-306, 1906.

[51] A. Einstein, "Berichtigung zu meiner arbeit: ,eine neue bestimmung der moleküldimensionen"," Annalen der Physik, vol. 339, no. 3, pp. 591-592, 1911. 
[52] S. H. Maron and P. E. Pierce, "Application of ree-eyring generalized flow theory to suspensions of spherical particles," Journal of Colloid Science, vol. 11, pp. 80-95, feb 1956.

[53] I. M. Krieger and T. J. Dougherty, "A mechanism for non-newtonian flow in suspensions of rigid spheres," Transactions of the Society of Rheology, vol. 3, pp. 137152, mar 1959.

[54] J. F. Morris and F. Boulay, "Curvilinear flows of noncolloidal suspensions: The role of normal stresses," Journal of Rheology, vol. 43, pp. 1213-1237, sep 1999.

[55] W. H. Herschel and R. Bulkley, "Konsistenzmessungen von gummi-benzollösungen,” Kolloid-Zeitschrift, vol. 39, pp. 291-300, aug 1926.

[56] E. Lindeberg and P. Bergmo, "The long-term fate of co2 injected into an aquifer," Greenhouse gas control technologies, vol. 1, pp. 489-494, 2003.

[57] U. Gregersen and P. Johannessen, "Distribution of the neogene utsira sand and the succeeding deposits in the viking graben area, north sea," Marine and Petroleum Geology, vol. 24, pp. 591-606, dec 2007.

[58] P. Zweigel, R. Arts, A. E. Lothe, and E. B. G. Lindeberg, "Reservoir geology of the utsira formation at the first industrial-scale underground CO2storage site (sleipner area, north sea)," Geological Society, London, Special Publications, vol. 233, no. 1, pp. 165-180, 2004.

[59] L. Cathles, Z. Su, and D. Chen, "The physics of gas chimney and pockmark formation, with implications for assessment of seafloor hazards and gas sequestration," Marine and Petroleum Geology, vol. 27, pp. 82-91, jan 2010.

[60] N. Shakhova, I. Semiletov, A. Salyuk, V. Yusupov, D. Kosmach, and O. Gustafsson, "Extensive methane venting to the atmosphere from sediments of the east siberian arctic shelf," Science, vol. 327, pp. 1246-1250, mar 2010.

[61] N. N. Romanovskii, H. W. Hubberten, A. V. Gavrilov, A. A. Eliseeva, and G. S. Tipenko, "Offshore permafrost and gas hydrate stability zone on the shelf of east siberian seas," Geo-Marine Letters, vol. 25, pp. 167-182, mar 2005.

[62] V. Soloviev, G. Ginzburg, E. Telepnev, and Y. Mikhaluk, "Cryothermia of gas hydrates in thearctic ocean (vniiokeangeologia, st. petersburg, russia," 1987.

[63] E. A. G. Schuur, J. G. Vogel, K. G. Crummer, H. Lee, J. O. Sickman, and T. E. Osterkamp, "The effect of permafrost thaw on old carbon release and net carbon exchange from tundra," Nature, vol. 459, pp. 556-559, may 2009. 
[64] K. M. Walter, S. A. Zimov, J. P. Chanton, D. Verbyla, and F. S. Chapin, "Methane bubbling from siberian thaw lakes as a positive feedback to climate warming," Nature, vol. 443, pp. 71-75, sep 2006.

[65] M. Hovland, R. Heggland, M. D. Vries, and T. Tjelta, "Unit-pockmarks and their potential significance for predicting fluid flow," Marine and Petroleum Geology, vol. 27, pp. 1190-1199, jun 2010.

[66] M.-J. Dalbe and R. Juanes, "Morphodynamics of fluid-fluid displacement in threedimensional deformable granular media," Physical Review Applied, vol. 9, feb 2018.

[67] G. Varas, G. Ramos, J.-C. Géminard, and V. Vidal, "Flow and fracture in watersaturated, unconstrained granular beds," Frontiers in Physics, vol. 3, jun 2015.

[68] Z. Sun and J. C. Santamarina, "Grain-displacive gas migration in fine-grained sediments," Journal of Geophysical Research: Solid Earth, vol. 124, pp. 2274-2285, mar 2019.

[69] B. P. Scandella, K. Delwiche, H. F. Hemond, and R. Juanes, "Persistence of bubble outlets in soft, methane-generating sediments," Journal of Geophysical Research: Biogeosciences, vol. 122, pp. 1298-1320, jun 2017.

[70] C. Chevalier, A. Lindner, and E. Clément, "Destabilization of a Saffman-Taylor fingerlike pattern in a granular suspension," Phys. Rev. Lett., vol. 99, no. 17, p. 174501, 2007.

[71] C. Chevalier, A. Lindner, M. Leroux, and E. Clément, "Morphodynamics during air injection into a confined granular suspension," J. NonNewton. Fluid, vol. 158, no. 1, pp. 63-72, 2009.

[72] G. Varas, J.-C. Géminard, and V. Vidal, "Air invasion in a granular layer immersed in a fluid: morphology and dynamics," Granular Matter, vol. 15, pp. 801-810, jul 2013.

[73] M. Trojer, M. L. Szulczewski, and R. Juanes, "Stabilizing fluid-fluid displacements in porous media through wettability alteration," Physical Review Applied, vol. 3, may 2015.

[74] B. Zhao, C. W. MacMinn, and R. Juanes, "Wettability control on multiphase flow in patterned microfluidics," Proceedings of the National Academy of Sciences, vol. 113, pp. 10251-10256, aug 2016. 
[75] R. Holtzman and E. Segre, "Wettability stabilizes fluid invasion into porous media via nonlocal, cooperative pore filling," Physical Review Letters, vol. 115, oct 2015.

[76] J. A. Eriksen, B. Marks, B. Sandnes, and R. Toussaint, "Bubbles breaking the wall: Two-dimensional stress and stability analysis," Physical Review E, vol. 91, may 2015 .

[77] S. Turkaya, R. Toussaint, F. K. Eriksen, M. Zecevic, G. Daniel, E. G. Flekkøy, and K. J. Måløy, "Bridging aero-fracture evolution with the characteristics of the acoustic emissions in a porous medium," Frontiers in Physics, vol. 3, sep 2015.

[78] F. K. Eriksen, R. Toussaint, A. L. Turquet, K. J. Måløy, and E. G. Flekkøy, "Pneumatic fractures in confined granular media," Physical Review E, vol. 95, no. 6, p. 062901, 2017.

[79] M. Niebling, R. Toussaint, E. G. Flekkøy, and K. J. Måløy, "Dynamic aerofracture of dense granular packings," Phys. Rev. E, vol. 86, p. 061315, 2012.

[80] A. Parmigiani, S. Faroughi, C. Huber, O. Bachmann, and Y. Su, "Bubble accumulation and its role in the evolution of magma reservoirs in the upper crust," Nature, vol. 532, pp. 492-495, apr 2016.

[81] K. V. Cashman, R. S. J. Sparks, and J. D. Blundy, "Vertically extensive and unstable magmatic systems: A unified view of igneous processes," Science, vol. 355, p. eaag3055, mar 2017.

[82] J. Suckale, T. Keller, K. V. Cashman, and P.-O. Persson, "Flow-to-fracture transition in a volcanic mush plug may govern normal eruptions at stromboli," Geophysical Research Letters, vol. 43, pp. 12,071-12,081, dec 2016.

[83] M. James, N. Bagdassarov, K. Müller, and H. Pinkerton, "Viscoelastic behaviour of basaltic lavas," Journal of Volcanology and Geothermal Research, vol. 132, pp. 99113, apr 2004.

[84] N. Bagdassarov and A. Dorfman, "Viscoelastic behavior of partially molten granites," Tectonophysics, vol. 290, pp. 27-45, may 1998.

[85] D. P. Hill, F. Pollitz, and C. Newhall, "Earthquake-volcano interactions," Physics Today, vol. 55, pp. 41-47, nov 2002.

[86] F. R. Fontaine, B. Ildefonse, and N. S. Bagdassarov, "Temperature dependence of shear wave attenuation in partially molten gabbronorite at seismic frequencies," Geophysical Journal International, vol. 163, pp. 1025-1038, dec 2005. 
[87] M. Pistone, L. Caricchi, P. Ulmer, E. Reusser, and P. Ardia, "Rheology of volatilebearing crystal mushes: Mobilization vs. viscous death," Chemical Geology, vol. 345, pp. 16-39, may 2013.

[88] J. Smith, "Textural evidence for dilatant (shear thickening) rheology of magma at high crystal concentrations," Journal of Volcanology and Geothermal Research, vol. 99, pp. 1-7, jun 2000.

[89] A. Srivastava, A. Majumdar, and B. S. Butola, "Improving the impact resistance of textile structures by using shear thickening fluids: A review," Critical Reviews in Solid State and Materials Sciences, vol. 37, pp. 115-129, apr 2012.

[90] Y. S. Lee, E. D. Wetzel, and N. J. Wagner, "The ballistic impact characteristics of kevlar woven fabrics impregnated with a colloidal shear thickening fluid," Journal of Materials Science, vol. 38, no. 13, pp. 2825-2833, 2003.

[91] N. Lee, Wagner and Wetzel, "Advanced body armor utilizing shear thickening fluids," in 23rd Army Science Conference, Orlando, FL, 2002.

[92] T. J. Kang, C. Y. Kim, and K. H. Hong, "Rheological behavior of concentrated silica suspension and its application to soft armor," Journal of Applied Polymer Science, vol. 124, pp. 1534-1541, oct 2011.

[93] B.-W. Lee and C.-G. Kim, "Computational analysis of shear thickening fluid impregnated fabrics subjected to ballistic impacts," Advanced Composite Materials, vol. 21, pp. 177-192, apr 2012.

[94] A. Majumdar, B. S. Butola, and A. Srivastava, "Development of soft composite materials with improved impact resistance using kevlar fabric and nano-silica based shear thickening fluid," Materials \& Design (1980-2015), vol. 54, pp. 295-300, feb 2014.

[95] Z. Lu, Z. Yuan, X. Chen, and J. Qiu, "Evaluation of ballistic performance of STF impregnated fabrics under high velocity impact," Composite Structures, p. 111208, jul 2019.

[96] Y. Wang, S. K. Li, and X. Y. Feng, "The ballistic performance of multi-layer kevlar fabrics impregnated with shear thickening fluids," Applied Mechanics and Materials, vol. 782, pp. 153-157, aug 2015.

[97] V. Tan, T. Tay, and W. Teo, "Strengthening fabric armour with silica colloidal suspensions," International Journal of Solids and Structures, vol. 42, pp. 1561-1576, mar 2005. 
[98] J. L. Park, B. I. Yoon, J. G. Paik, and T. J. Kang, "Ballistic performance of paramid fabrics impregnated with shear thickening fluid part $\mathrm{i}$ - effect of laminating sequence," Textile Research Journal, vol. 82, pp. 527-541, sep 2011.

[99] J. L. Park, B. I. Yoon, J. G. Paik, and T. J. Kang, "Ballistic performance of p-aramid fabrics impregnated with shear thickening fluid part II - effect of fabric count and shot location," Textile Research Journal, vol. 82, pp. 542-557, feb 2012.

[100] R. J. Rabb and E. P. Fahrenthold, "Evaluation of shear-thickening-fluid kevlar for large-fragment-containment applications," Journal of Aircraft, vol. 48, pp. 230234, jan 2011.

[101] C. G. Kim, I. J. Kim, G. Lim, and B. I. Yoon, "The ballistic impact characteristics of woven fabrics impregnated with a colloidal suspension and flattened rolls," Advances in Science and Technology, vol. 71, pp. 74-79, oct 2010.

[102] Y. Park, Y. Kim, A. H. Baluch, and C.-G. Kim, "Empirical study of the high velocity impact energy absorption characteristics of shear thickening fluid (STF) impregnated kevlar fabric," International Journal of Impact Engineering, vol. 72, pp. 67-74, oct 2014.

[103] Y. Park, Y. Kim, A. H. Baluch, and C.-G. Kim, "Numerical simulation and empirical comparison of the high velocity impact of STF impregnated kevlar fabric using friction effects," Composite Structures, vol. 125, pp. 520-529, jul 2015.

[104] Y. T. Hu, P. Boltenhagen, E. Matthys, and D. J. Pine, "Shear thickening in lowconcentration solutions of wormlike micelles. II. slip, fracture, and stability of the shear-induced phase," Journal of Rheology, vol. 42, pp. 1209-1226, sep 1998.

[105] M. E. Cates, M. D. Haw, and C. B. Holmes, "Dilatancy, jamming, and the physics of granulation," Journal of Physics: Condensed Matter, vol. 17, pp. S2517-S2531, jun 2005.

[106] J. J. Stickel and R. L. Powell, "FLUID MECHANICS AND RHEOLOGY OF DENSE SUSPENSIONS," Annual Review of Fluid Mechanics, vol. 37, pp. 129149, jan 2005.

[107] M. Smith, R. Besseling, M. Cates, and V. Bertola, "Dilatancy in the flow and fracture of stretched colloidal suspensions," Nature Communications, vol. 1, p. 114, nov 2010.

[108] M. M. Denn and J. F. Morris, "Rheology of non-brownian suspensions," Annual Review of Chemical and Biomolecular Engineering, vol. 5, pp. 203-228, jun 2014. 
[109] S. Dagois-Bohy, S. Hormozi, É. Guazzelli, and O. Pouliquen, "Rheology of dense suspensions of non-colloidal spheres in yield-stress fluids," Journal of Fluid Mechanics, vol. 776, jul 2015.

[110] N. C. Crawford, L. B. Popp, K. E. Johns, L. M. Caire, B. N. Peterson, and M. W. Liberatore, "Shear thickening of corn starch suspensions: Does concentration matter?," Journal of Colloid and Interface Science, vol. 396, pp. 83-89, apr 2013.

[111] B. L. Johnson, M. R. Holland, J. G. Miller, and J. I. Katz, "Ultrasonic attenuation and speed of sound of cornstarch suspensions," The Journal of the Acoustical Society of America, vol. 133, pp. 1399-1403, mar 2013.

[112] E. Brown and H. M. Jaeger, "Shear thickening in concentrated suspensions: phenomenology, mechanisms and relations to jamming," Reports on Progress in Physics, vol. 77, p. 046602, apr 2014.

[113] E. Brown and H. M. Jaeger, "Dynamic jamming point for shear thickening suspensions," Physical Review Letters, vol. 103, aug 2009.

[114] E. Brown and H. M. Jaeger, "The role of dilation and confining stresses in shear thickening of dense suspensions," Journal of Rheology, vol. 56, pp. 875-923, jul 2012 .

[115] A. Fall, F. Bertrand, G. Ovarlez, and D. Bonn, "Shear thickening of cornstarch suspensions," Journal of Rheology, vol. 56, pp. 575-591, may 2012.

[116] R. Maharjan and E. Brown, "Giant deviation of a relaxation time from generalized newtonian theory in discontinuous shear thickening suspensions," Physical Review Fluids, vol. 2, dec 2017.

[117] J. J. S. Jerome, N. Vandenberghe, and Y. Forterre, "Unifying impacts in granular matter from quicksand to cornstarch," Physical Review Letters, vol. 117, aug 2016.

[118] R. Maharjan, S. Mukhopadhyay, B. Allen, T. Storz, and E. Brown, “Constitutive relation for the system-spanning dynamically jammed region in response to impact of cornstarch and water suspensions," Physical Review E, vol. 97, may 2018.

[119] A. Fall, A. Lemaître, and G. Ovarlez, "Discontinuous shear thickening in cornstarch suspensions," EPJ Web of Conferences, vol. 140, p. 09001, 2017.

[120] E. Han, I. R. Peters, and H. M. Jaeger, "High-speed ultrasound imaging in dense suspensions reveals impact-activated solidification due to dynamic shear jamming," Nature Communications, vol. 7, p. 12243, jul 2016. 
[121] E. Han, M. Wyart, I. R. Peters, and H. M. Jaeger, "Shear fronts in shear-thickening suspensions," Physical Review Fluids, vol. 3, jul 2018.

[122] I. R. Peters and H. M. Jaeger, "Quasi-2d dynamic jamming in cornstarch suspensions: visualization and force measurements," Soft Matter, vol. 10, no. 34, pp. 6564-6570, 2014.

[123] I. R. Peters, S. Majumdar, and H. M. Jaeger, "Direct observation of dynamic shear jamming in dense suspensions," Nature, vol. 532, pp. 214-217, apr 2016.

[124] S. R. Waitukaitis and H. M. Jaeger, "Impact-activated solidification of dense suspensions via dynamic jamming fronts," Nature, vol. 487, pp. 205-209, jul 2012.

[125] M. Hermes, B. M. Guy, W. C. K. Poon, G. Poy, M. E. Cates, and M. Wyart, "Unsteady flow and particle migration in dense, non-brownian suspensions," Journal of Rheology, vol. 60, pp. 905-916, sep 2016.

[126] O. Ozgen, M. Kallmann, and E. Brown, "Simulating the dynamic behavior of shear thickening fluids," http://arxiv.org/abs/1510.09069v1, oct 2015.

[127] A. S. Baumgarten and K. Kamrin, "A general constitutive model for dense, fineparticle suspensions validated in many geometries," Proceedings of the National Academy of Sciences, p. 201908065, sep 2019.

[128] W. J. Frith, P. d'Haene, R. Buscall, and J. Mewis, "Shear thickening in model suspensions of sterically stabilized particles," Journal of Rheology, vol. 40, pp. 531548, jul 1996.

[129] B. J. Maranzano and N. J. Wagner, "The effects of particle size on reversible shear thickening of concentrated colloidal dispersions," The Journal of Chemical Physics, vol. 114, pp. 10514-10527, jun 2001.

[130] S. S. Shenoy and N. J. Wagner, "Influence of medium viscosity and adsorbed polymer on the reversible shear thickening transition in concentrated colloidal dispersions," Rheologica Acta, vol. 44, pp. 360-371, dec 2004.

[131] R. Hoffman, "Discontinuous and dilatant viscosity behavior in concentrated suspensions. II. theory and experimental tests," Journal of Colloid and Interface Science, vol. 46, pp. 491-506, mar 1974.

[132] R. G. Egres and N. J. Wagner, "The rheology and microstructure of acicular precipitated calcium carbonate colloidal suspensions through the shear thickening transition," Journal of Rheology, vol. 49, pp. 719-746, may 2005. 
[133] R. G. Egres, F. Nettesheim, and N. J. Wagner, "Rheo-SANS investigation of acicular-precipitated calcium carbonate colloidal suspensions through the shear thickening transition," Journal of Rheology, vol. 50, pp. 685-709, sep 2006.

[134] B. J. Maranzano and N. J. Wagner, "Flow-small angle neutron scattering measurements of colloidal dispersion microstructure evolution through the shear thickening transition," The Journal of Chemical Physics, vol. 117, pp. 10291-10302, dec 2002.

[135] J. F. Brady and G. Bossis, "The rheology of concentrated suspensions of spheres in simple shear flow by numerical simulation," Journal of Fluid Mechanics, vol. 155, p. 105, jun 1985 .

[136] N. J. Wagner and J. F. Brady, "Shear thickening in colloidal dispersions," Physics Today, vol. 62, pp. 27-32, oct 2009.

[137] X. Cheng, J. H. McCoy, J. N. Israelachvili, and I. Cohen, "Imaging the microscopic structure of shear thinning and thickening colloidal suspensions," Science, vol. 333, pp. 1276-1279, sep 2011.

[138] H. M. Jaeger, S. R. Nagel, and R. P. Behringer, "Granular solids, liquids, and gases," Reviews of Modern Physics, vol. 68, pp. 1259-1273, oct 1996.

[139] O. Reynolds, "On the dilatancy of media composed of rigid particles in contact. with experimental illustrations," The London, Edinburgh, and Dublin Philosophical Magazine and Journal of Science, vol. 20, pp. 469-481, dec 1885.

[140] G. Y. Onoda and E. G. Liniger, "Random loose packings of uniform spheres and the dilatancy onset," Physical Review Letters, vol. 64, pp. 2727-2730, may 1990.

[141] A. B. Metzner and M. Whitlock, "Flow behavior of concentrated (dilatant) suspensions," Transactions of the Society of Rheology, vol. 2, pp. 239-254, mar 1958.

[142] C. B. Holmes, M. Fuchs, and M. E. Cates, "Jamming transitions in a schematic model of suspension rheology," Europhysics Letters (EPL), vol. 63, pp. 240-246, jul 2003.

[143] M. Trulsson, B. Andreotti, and P. Claudin, "Transition from the viscous to inertial regime in dense suspensions," Physical Review Letters, vol. 109, sep 2012.

[144] R. Seto, R. Mari, J. F. Morris, and M. M. Denn, "Discontinuous shear thickening of frictional hard-sphere suspensions," Physical Review Letters, vol. 111, nov 2013. 
[145] R. Mari, R. Seto, J. F. Morris, and M. M. Denn, "Shear thickening, frictionless and frictional rheologies in non-brownian suspensions," Journal of Rheology, vol. 58, pp. 1693-1724, nov 2014.

[146] M. Wyart and M. Cates, "Discontinuous shear thickening without inertia in dense non-brownian suspensions," Physical Review Letters, vol. 112, mar 2014.

[147] J. R. Melrose and R. C. Ball, "The pathological behaviour of sheared hard spheres with hydrodynamic interactions," Europhysics Letters (EPL), vol. 32, pp. 535-540, nov 1995.

[148] N. Fernandez, R. Mani, D. Rinaldi, D. Kadau, M. Mosquet, H. Lombois-Burger, J. Cayer-Barrioz, H. J. Herrmann, N. D. Spencer, and L. Isa, "Microscopic mechanism for shear thickening of non-brownian suspensions," Physical Review Letters, vol. 111, sep 2013.

[149] C. Heussinger, "Shear thickening in granular suspensions: Interparticle friction and dynamically correlated clusters," Physical Review E, vol. 88, nov 2013.

[150] A. Vázquez-Quesada, N. J. Wagner, and M. Ellero, "Planar channel flow of a discontinuous shear-thickening model fluid: Theory and simulation," Physics of Fluids, vol. 29, p. 103104, oct 2017.

[151] J. Comtet, G. Chatté, A. Niguès, L. Bocquet, A. Siria, and A. Colin, "Pairwise frictional profile between particles determines discontinuous shear thickening transition in non-colloidal suspensions," Nature Communications, vol. 8, p. 15633, may 2017.

[152] A. W. Alexander, B. Chaudhuri, A. Faqih, F. J. Muzzio, C. Davies, and M. S. Tomassone, "Avalanching flow of cohesive powders," Powder Technology, vol. 164, pp. 13-21, may 2006.

[153] A. Castellanos, J. M. Valverde, and M. A. S. Quintanilla, "Fine cohesive powders in rotating drums: Transition from rigid-plastic flow to gas-fluidized regime," Physical Review E, vol. 65, jun 2002.

[154] S.-Y. Lim, J. Davidson, R. Forster, D. Parker, D. Scott, and J. Seville, "Avalanching of granular material in a horizontal slowly rotating cylinder: PEPT studies," Powder Technology, vol. 138, pp. 25-30, nov 2003.

[155] J. Mellmann, "The transverse motion of solids in rotating cylinders-forms of motion and transition behavior," Powder Technology, vol. 118, pp. 251-270, aug 2001. 
[156] C. Clavaud, A. Bérut, B. Metzger, and Y. Forterre, "Revealing the frictional transition in shear-thickening suspensions," Proceedings of the National Academy of Sciences, vol. 114, pp. 5147-5152, may 2017.

[157] S. C. du Pont, P. Gondret, B. Perrin, and M. Rabaud, "Granular avalanches in fluids," Physical Review Letters, vol. 90, jan 2003.

[158] M. Pailha, M. Nicolas, and O. Pouliquen, "Initiation of underwater granular avalanches: Influence of the initial volume fraction," Physics of Fluids, vol. 20, p. 111701, nov 2008.

[159] F. Boyer, É. Guazzelli, and O. Pouliquen, "Unifying suspension and granular rheology," Physical Review Letters, vol. 107, oct 2011.

[160] É. Guazzelli and O. Pouliquen, "Rheology of dense granular suspensions," Journal of Fluid Mechanics, vol. 852, aug 2018.

[161] H. Zhao and J. V. Maher, "Associating-polymer effects in a hele-shaw experiment," Physical Review E, vol. 47, pp. 4278-4283, jun 1993.

[162] C. Ligoure and S. Mora, "Fractures in complex fluids: the case of transient networks," Rheologica Acta, vol. 52, pp. 91-114, jan 2013.

[163] J. F. Hutton, "Fracture of liquids in shear,” Nature, vol. 203, pp. 177-177, jul 1964.

[164] J.-F. Berret and Y. Séréro, "Evidence of shear-induced fluid fracture in telechelic polymer networks," Physical Review Letters, vol. 87, jul 2001.

[165] P. J. Skrzeszewska, J. Sprakel, F. A. de Wolf, R. Fokkink, M. A. C. Stuart, and J. van der Gucht, "Fracture and self-healing in a well-defined self-assembled polymer network," Macromolecules, vol. 43, pp. 3542-3548, apr 2010.

[166] J. Ignés-Mullol, H. Zhao, and J. V. Maher, "Velocity fluctuations of fracturelike disruptions of associating polymer solutions," Physical Review E, vol. 51, pp. 13381343, feb 1995.

[167] D. H. Vlad, J. Ignes-Mullol, and J. V. Maher, "Velocity-jump instabilities in heleshaw flow of associating polymer solutions," Physical Review E, vol. 60, pp. 44234430, oct 1999.

[168] S. Mora and M. Manna, "Saffman-taylor instability of viscoelastic fluids: From viscous fingering to elastic fractures," Physical Review E, vol. 81, feb 2010.

[169] Y. Mulla, G. Oliveri, J. T. Overvelde, and G. H. Koenderink, "Crack initiation in viscoelastic materials," Physical Review Letters, vol. 120, jun 2018. 
[170] H. Tabuteau, S. Mora, G. Porte, M. Abkarian, and C. Ligoure, "Microscopic mechanisms of the brittleness of viscoelastic fluids," Physical Review Letters, vol. 102, apr 2009.

[171] T. Tixier, H. Tabuteau, A. Carrière, L. Ramos, and C. Ligoure, "Transition from "brittle" to "ductile" rheological behavior by tuning the morphology of selfassembled networks," Soft Matter, vol. 6, no. 12, p. 2699, 2010.

[172] H. Tabuteau, S. Mora, M. Ciccotti, C.-Y. Hui, and C. Ligoure, "Propagation of a brittle fracture in a viscoelastic fluid," Soft Matter, vol. 7, no. 19, p. 9474, 2011.

[173] E. Lemaire, P. Levitz, G. Daccord, and H. V. Damme, "From viscous fingering to viscoelastic fracturing in colloidal fluids," Physical Review Letters, vol. 67, pp. 2009-2012, oct 1991.

[174] N. Kagei, D. Kanie, and M. Kawaguchi, "Viscous fingering in shear thickening silica suspensions," Physics of Fluids, vol. 17, p. 054103, may 2005.

[175] J. Cras, C. Rowe-Taitt, D. Nivens, and F. Ligler, "Comparison of chemical cleaning methods of glass in preparation for silanization," Biosensors and Bioelectronics, vol. 14, no. 8, pp. $683-688,1999$.

[176] F. A. L. Dullien, Porous media: fluid transport and pore structure. Academic press, 2012

[177] J. Blaber, B. Adair, and A. Antoniou, "Ncorr: Open-source 2d digital image correlation matlab software," Experimental Mechanics, vol. 55, pp. 1105-1122, Jul 2015.

[178] H. Shin and J. C. Santamarina, "Fluid-driven fractures in uncemented sediments: underlying particle-level processes," Earth Planet. Sc. Lett., vol. 299, no. 1, pp. 180-189, 2010.

[179] J.-H. Choi, Y. Seol, R. Boswell, and R. Juanes, "X-ray computed-tomography imaging of gas migration in water-saturated sediments: from capillary invasion to conduit opening," Geophys. Res. Lett., vol. 38, no. 17, 2011.

[180] K. E. Fauria and A. W. Rempel, "Gas invasion into water-saturated, unconsolidated porous media: implications for gas hydrate reservoirs," Earth Planet. Sc. Lett., vol. 312, no. 1, pp. 188-193, 2011.

[181] L. Goehring, R. Conroy, A. Akhter, W. J. Clegg, and A. F. Routh, "Evolution of mud-crack patterns during repeated drying cycles," Soft Matter, vol. 6, no. 15, pp. 3562-3567, 2010 . 
[182] S. Kitsunezaki, "Crack growth in drying paste," Adv. Powder Technol., vol. 22, no. 3, pp. 311-318, 2011.

[183] R. Weinberger, "Evolution of polygonal patterns in stratified mud during desiccation: the role of flaw distribution and layer boundaries," Geol. Soc. Am. Bull., vol. 113, no. 1, pp. 20-31, 2001.

[184] A. K. Jain and R. Juanes, "Preferential mode of gas invasion in sediments: Grainscale mechanistic model of coupled multiphase fluid flow and sediment mechanics," Journal of Geophysical Research, vol. 114, aug 2009.

[185] A. Lindoo, J. Larsen, K. Cashman, and J. Oppenheimer, "Crystal controls on permeability development and degassing in basaltic andesite magma," Geology, vol. 45, pp. 831-834, jul 2017.

[186] A. Rogala, J. Krzysiek, M. Bernaciak, and H. J., "Non-aqueous fracturing technologies for shale gas recovery," Physicochem. Probl. Miner. Process., vol. 49, no. 1, pp. 313-322, 2013.

[187] H.-J. Butt, K. Graf, and M. Kappl, Physics and chemistry of interfaces. John Wiley \& Sons, 2006.

[188] M. Urbakh, J. Klafter, D. Gourdon, and J. Israelachvili, "The nonlinear nature of friction," Nature, vol. 430, pp. 525-528, jul 2004.

[189] W. F. Brace and J. D. Byerlee, "Stick-slip as a mechanism for earthquakes," Science, vol. 153, pp. 990-992, aug 1966.

[190] R. Fischer, P. Gondret, and M. Rabaud, "Transition by intermittency in granular matter: From discontinuous avalanches to continuous flow," Physical Review Letters, vol. 103, sep 2009.

[191] C.-H. Liu, S. R. Nagel, D. A. Schecter, S. N. Coppersmith, S. Majumdar, O. Narayan, and T. A. Witten, "Force fluctuations in bead packs," Science, vol. 269, no. 5223, pp. 513-515, 1995.

[192] T. S. Majmudar and R. P. Behringer, "Contact force measurements and stressinduced anisotropy in granular materials," Nature, vol. 435, no. 7045, pp. 1079$1082,2005$.

[193] A. J. Liu and S. R. Nagel, "Jamming is not just cool any more," Nature, vol. 396, no. 6706, pp. 21-22, 1998.

[194] V. Trappe, V. Prasad, L. Cipelletti, P. N. Segre, and D. A. Weitz, "Jamming phase diagram for attractive particles," Nature, vol. 411, no. 6839, pp. 772-775, 2001. 
[195] M. Van Hecke, "Jamming of soft particles: geometry, mechanics, scaling and isostaticity," J. Phys.-Condens. Mat., vol. 22, no. 3, p. 033101, 2010.

[196] F. K. Eriksen, R. Toussaint, K. J. Måløy, and E. G. Flekkøy, "Invasion patterns during two-phase flow in deformable porous media," Front Phys, vol. 3, no. 81, 2015.

[197] X.-Z. Kong, W. Kinzelbach, and F. Stauffer, "Morphodynamics during air injection into water-saturated movable spherical granulates," Chemical Engineering Science, vol. 65, pp. 4652-4660, aug 2010.

[198] G. Mason and D. W. Mellor, "Simulation of drainage and imbibition in a random packing of equal spheres," J. Colloid Interf. Sci., vol. 176, no. 1, pp. 214-225, 1995.

[199] R. Holtzman and R. Juanes, "Crossover from fingering to fracturing in deformable disordered media," Phys. Rev. E, vol. 82, p. 046305, Oct 2010.

[200] G. Dumazer, B. Sandnes, M. Ayaz, K. J. Måløy, and E. G. Flekkøy, "Frictional fluid dynamics and plug formation in multiphase millifluidic flow," Phys. Rev. Lett., vol. 117, p. 028002, 2016.

[201] J. Bear, Dynamics of fluids in porous media. Elsevier, 1972.

[202] A. Groisman and E. Kaplan, "An experimental study of cracking induced by desiccation," Europhysics Letters (EPL), vol. 25, pp. 415-420, feb 1994.

[203] R. Barati and J.-T. Liang, "A review of fracturing fluid systems used for hydraulic fracturing of oil and gas wells," Journal of Applied Polymer Science, vol. 131, apr 2014.

[204] L. Sair and W. R. Fetzer, "Water sorption by starches," Industrial \& Engineering Chemistry, vol. 36, pp. 205-208, mar 1944.

[205] N. N. Hellman and E. H. Melvin, "Surface area of starch and its role in water sorption," Journal of the American Chemical Society, vol. 72, pp. 5186-5188, nov 1950.

[206] A. J. H. John J Duffy and S. H. Murphy, "Simple method for determining stress and strain constants for non-standard measuring systems on a rotational rheometer," 2015.

[207] R. H. Ewoldt, M. T. Johnston, and L. M. Caretta, "Experimental challenges of shear rheology: How to avoid bad data," in Complex Fluids in Biological Systems, pp. 207-241, Springer New York, oct 2014. 
[208] M. Cross and A. Kaye, "Simple procedures for obtaining viscosity/shear rate data from a parallel disc viscometer," Polymer, vol. 28, pp. 435-440, mar 1987.

[209] A. Fall, N. Huang, F. Bertrand, G. Ovarlez, and D. Bonn, "Shear thickening of cornstarch suspensions as a reentrant jamming transition," Physical Review Letters, vol. 100, jan 2008 .

[210] E. E. B. White, M. Chellamuthu, and J. P. Rothstein, "Extensional rheology of a shear-thickening cornstarch and water suspension," Rheologica Acta, vol. 49, pp. 119-129, dec 2009.

[211] M. Roché, H. Kellay, and H. A. Stone, "Heterogeneity and the role of normal stresses during the extensional thinning of non-brownian shear-thickening fluids," Physical Review Letters, vol. 107, sep 2011.

[212] S. von Kann, J. H. Snoeijer, D. Lohse, and D. van der Meer, "Nonmonotonic settling of a sphere in a cornstarch suspension," Physical Review E, vol. 84, dec 2011.

[213] A. Singh, R. Mari, M. M. Denn, and J. F. Morris, "A constitutive model for simple shear of dense frictional suspensions," Journal of Rheology, vol. 62, pp. 457-468, mar 2018.

[214] J. M. Campbell, D. Ozturk, and B. Sandnes, "Gas-driven fracturing of saturated granular media," Physical Review Applied, vol. 8, dec 2017.

[215] D. Ozturk and B. Sandnes, "A Study Of Three-Phase Fracturing In Granular Media Using High-Speed Imaging,” Journal of Porous Media, vol. 22, no. 8, pp. 9871000, 2019. 